\title{
Asymmetric Total Synthesis of Arcutinidine, Arcutinine, and Arcutine
}

\author{
Shupeng Zhou, ${ }^{\dagger}$ Kaifu Xia ${ }^{\dagger}$ Xuebing Leng, ${ }^{\dagger}$ and Ang $\mathrm{Li}^{*},{ }^{\dagger}$ \\ ${ }^{\dagger}$ State Key Laboratory of Bioorganic and Natural Products Chemistry, Center for Excellence in Molecular \\ Synthesis, Shanghai Institute of Organic Chemistry, University of Chinese Academy of Sciences, Chinese \\ Academy of Sciences, 345 Lingling Road, Shanghai 200032, China \\ ${ }^{\ddagger}$ State Key Laboratory of Organometallic Chemistry, Shanghai Institute of Organic Chemistry, Chinese \\ Academy of Sciences, 345 Lingling Road, Shanghai 200032, China \\ E-mail: ali@sioc.ac.cn
}

I Experimental Procedures and Spectroscopic Data of Compounds

II HPLC Traces for Measuring Enantiomeric Excess

III $\quad{ }^{1} \mathrm{H}$ and ${ }^{13} \mathrm{C}$ NMR Spectra of Compounds

IV Comparison of the Spectroscopic Data of Authentic and Synthetic Arcutinidine

V Circular Dichroism Spectra of Synthetic Arcutinidine and Arcutine

VI References 


\section{Experimental Procedures and Spectroscopic Data of Compounds}

General Procedures. All reactions were carried out under an argon atmosphere with dry solvents under anhydrous conditions, unless otherwise noted. Tetrahydrofuran (THF) was distilled immediately before use from sodium-benzophenoneketyl. Methylene chloride $\left(\mathrm{CH}_{2} \mathrm{Cl}_{2}\right), N, N$-dimethylformamide (DMF), hexamethylphosphoramide (HMPA), tert-butanol $(t-\mathrm{BuOH})$, diisopropylamine $\left(i\right.$ - $\left.\mathrm{Pr}_{2} \mathrm{NH}\right)$, triethylamine $\left(\mathrm{Et}_{3} \mathrm{~N}\right)$, and pyridine were distilled from calcium hydride and stored under an argon atmosphere. Methanol $(\mathrm{MeOH})$ and ethanol $(\mathrm{EtOH})$ was distilled from magnesium and stored under an argon atmosphere. Reagents were purchased at the highest commercial quality and used without further purification, unless otherwise stated. Lipase acrylic resin from Candida antarctica ( $\geq 5,000 \mathrm{U} / \mathrm{g}$, recombinant, expressed in Aspergillus niger) was purchased from Sigma-Aldrich. Solvents for chromatography were used as supplied by Adamas-beta ${ }^{\circledR}$. Reactions were monitored by thin layer chromatography (TLC) carried out on MilliporeSigma glass TLC plates (silica gel 60 coated with $\mathrm{F}_{254}$, $250 \mu \mathrm{m}$ ) using UV light for visualization and aqueous ammonium cerium nitrate/ammonium molybdate or basic aqueous potassium permanganate as developing agent. SiliaFlash ${ }^{\circledR}$ P60silica gel (particle size: 40-63 $\mu \mathrm{m}$, pore size: $60 \AA$ A) was used for flash column chromatography. NMR spectra were recorded on a Bruker Avance III $400 \mathrm{MHz}$, an Agilent DD2 $500 \mathrm{MHz}$, or a Bruker Avance III HD 600 MHz NMR spectrometer. The spectra were calibrated by using residual undeuterated solvents (for ${ }^{1} \mathrm{H}$ NMR) and deuterated solvents (for ${ }^{13} \mathrm{C}$ NMR) as internal references: undeuterated chloroform $\left(\delta_{\mathrm{H}}=7.26 \mathrm{ppm}\right)$ and $\mathrm{CDCl}_{3}\left(\delta_{\mathrm{C}}=77.16 \mathrm{ppm}\right) ;$ undeuterated methanol $\left(\delta_{\mathrm{H}}=3.31 \mathrm{ppm}\right)$ and methanol- $\mathrm{d}_{4}\left(\delta_{\mathrm{C}}=49.00 \mathrm{ppm}\right) ;$ undeuterated acetone $\left(\delta_{\mathrm{H}}=2.05 \mathrm{ppm}\right)$ and acetone $-\mathrm{d}_{6}\left(\delta_{\mathrm{C}}=29.84 \mathrm{ppm}\right)$. The following abbreviations are used to designate multiplicities: $\mathrm{s}=$ singlet, $\mathrm{d}=$ doublet, $\mathrm{t}=$ triplet, $\mathrm{q}=$ quartet, $\mathrm{m}=$ multiplet, $\mathrm{br}=$ broad. IR spectra were recorded on a Thermo Scientific Nicolet 380 FT-IR spectrometer. Melting points (m.p.) are uncorrected and were recorded on an SGW X-4 apparatus. High-resolution mass spectra (HRMS) were recorded on a Bruker maXis $4 \mathrm{G}$ mass spectrometer. 


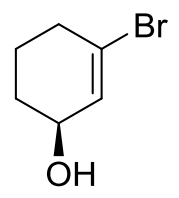

13

Enantioenriched alcohol 13: The preparation of this compound through enzymatic kinetic resolution has been reported in the literature. ${ }^{1}$ We developed an improved protocol to obtain highly enantioenriched 13. Racemic $\mathbf{1 3}$ was prepared from commercially available 1,3-cyclohexanedione through a two-step sequence. ${ }^{\mathrm{b}, 2}$ To a stirred solution of racemic $\mathbf{1 3}$ (40.0 g, $226 \mathrm{mmol}, 1.0$ equiv) in $t$-BuOMe $(160 \mathrm{~mL})$ were sequentially added vinyl acetate $(23.4 \mathrm{~g}, 25.0 \mathrm{~mL}, 272 \mathrm{mmol}, 1.2 \mathrm{equiv})$ and lipase acrylic resin from Candida antarctica $(400 \mathrm{mg})$ at $22{ }^{\circ} \mathrm{C} .{ }^{3}$ The resultant mixture was allowed to stir at that temperature for $58 \mathrm{~h}$ before it was filtered. The filtrate was concentrated under vacuum, and the residue was purified by flash column chromatography with EtOAc/petroleum ether $(1: 5 \rightarrow 1: 2)$ to give enantioenriched alcohol $13(16.8 \mathrm{~g}, 42 \%)$ as a pale yellow oil. The ee of this sample was determined to be $99.7 \%$ by HPLC (vide infra). 13: $[\alpha]_{\mathrm{D}}^{25}=-39.1\left(c=1.0\right.$ in $\left.\mathrm{CHCl}_{3}\right)$. The spectroscopic data and physical properties of $\mathbf{1 3}$ are identical to those reported in the literature. ${ }^{1}$

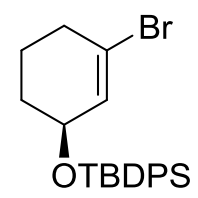

14

Silyl ether 14: To a stirred solution of enantioenriched alcohol 13 (25.6 g, 145 mmol, 1.0 equiv) in DMF (30 mL) were sequentially added imidazole (14.8 g, $217 \mathrm{mmol}, 1.5$ equiv) and TBDPSCl (42.3 g, $40.0 \mathrm{~mL}, 154 \mathrm{mmol}, 1.1$ equiv) at $22{ }^{\circ} \mathrm{C}$. The mixture was allowed to stir at that temperature for $8 \mathrm{~h}$ before saturated aq. $\mathrm{NaHCO}_{3}(300 \mathrm{~mL})$ was added. The resultant mixture was extracted with petroleum ether $(3 \times 400 \mathrm{~mL})$. The combined organic phases were washed with brine $(300 \mathrm{~mL})$, dried over anhydrous $\mathrm{Na}_{2} \mathrm{SO}_{4}$, filtered, and concentrated under vacuum. The residue was subjected to flash column chromatography for purification using EtOAc/petroleum ether (1:100) as eluent to give silyl ether 14 $(56.2 \mathrm{~g}, 93 \%)$ as a white powder. 14: $R_{\mathrm{f}}=0.27$ (silica, petroleum ether); $[\alpha]_{\mathrm{D}}^{25}=-83.9(c=1.0$ in $\mathrm{CHCl}_{3}$ ); IR (film): $v_{\max }=3070,3042,2930,2857,1646,1427,1361,1111,1022,972,701 \mathrm{~cm}^{-1} ;{ }^{1} \mathrm{H}$ 
NMR (500 MHz, $\left.\mathrm{CDCl}_{3}\right): \delta=7.70-7.63(\mathrm{~m}, 4 \mathrm{H}), 7.51-7.31(\mathrm{~m}, 6 \mathrm{H}), 5.98-5.93(\mathrm{~m}, 1 \mathrm{H}), 4.24-4.18$

(m, $1 \mathrm{H}), 2.51-2.38(\mathrm{~m}, 1 \mathrm{H}), 2.36-2.23(\mathrm{~m}, 1 \mathrm{H}), 1.97-1.83(\mathrm{~m}, 1 \mathrm{H}), 1.70-1.60$ (m, $2 \mathrm{H}), 1.59-1.48$

(m, $1 \mathrm{H}), 1.06$ (s, $9 \mathrm{H}) \mathrm{ppm} ;{ }^{13} \mathrm{C}$ NMR $\left(101 \mathrm{MHz}, \mathrm{CDCl}_{3}\right): \delta=135.93,135.90,134.20,134.11,132.27$, 129.83, 129.82, 127.77, 127.75, 125.90, 68.56, 35.32, 30.89, 27.07, 20.83, 19.31 ppm; HRMS $(\mathrm{m} / \mathrm{z}):$ : $\mathrm{M}$ $+\mathrm{Na}]^{+}$calcd for $\mathrm{C}_{22} \mathrm{H}_{27} \mathrm{OBrSiNa}{ }^{+}$437.0907, found 437.0906.

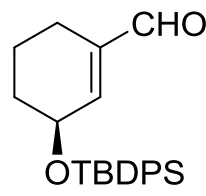

12

$\boldsymbol{\alpha}, \boldsymbol{\beta}$-Unsaturated enal 12: To a stirred solution of silyl ether $\mathbf{1 4}(15.8 \mathrm{~g}, 38.0 \mathrm{mmol}, 1.0$ equiv) in THF $(200 \mathrm{~mL})$ was added $t$ - $\mathrm{BuLi}\left(50.0 \mathrm{~mL}, 80.0 \mathrm{mmol}, 1.6 \mathrm{M}\right.$ in pentane, 2.1 equiv) at $-78{ }^{\circ} \mathrm{C}$. The mixture was allowed to stir at that temperature for $30 \mathrm{~min}$ before DMF (6.04 g, $6.40 \mathrm{~mL}, 82.6 \mathrm{mmol}, 2.2$ equiv) was added. The resultant mixture was allowed to warm to $22{ }^{\circ} \mathrm{C}$ and stir at that temperature for $30 \mathrm{~min}$. Saturated aq. $\mathrm{NaHCO}_{3}(200 \mathrm{~mL})$ was then added, and the mixture was extracted with EtOAc $(3 \times 300$ $\mathrm{mL})$. The combined organic phases were washed with brine $(200 \mathrm{~mL})$, dried over anhydrous $\mathrm{Na}_{2} \mathrm{SO}_{4}$, and filtered. The volatiles were removed under vacuum, and the residue was purified by flash column chromatography with EtOAc/petroleum ether $(1: 50 \rightarrow 1: 30)$ to give $\alpha, \beta$-unsaturated enal $12(12.6 \mathrm{~g}$, 91\%). 12: $R_{\mathrm{f}}=0.35$ (silica, EtOAc:petroleum ether 1:40); $[\alpha]_{\mathrm{D}}^{25}=-97.2\left(c=1.0\right.$ in $\left.\mathrm{CHCl}_{3}\right) ; \mathrm{IR}($ film): $v_{\max }=3070,3049,2931,2857,2708,1690,1471,1427,1172,1110,1080,701 \mathrm{~cm}^{-1} ;{ }^{1} \mathrm{H}$ NMR $(400$ $\left.\mathrm{MHz}, \mathrm{CDCl}_{3}\right): \delta=9.37-9.34(\mathrm{~m}, 1 \mathrm{H}), 7.73-7.66(\mathrm{~m}, 4 \mathrm{H}), 7.49-7.43(\mathrm{~m}, 2 \mathrm{H}), 7.43-7.37(\mathrm{~m}, 4 \mathrm{H})$, 6.51-6.46(m, $1 \mathrm{H}), 4.48-4.39(\mathrm{~m}, 1 \mathrm{H}), 2.19-2.01(\mathrm{~m}, 2 \mathrm{H}), 1.88-1.74(\mathrm{~m}, 2 \mathrm{H}), 1.74-1.62(\mathrm{~m}, 1 \mathrm{H})$, $1.51-1.36(\mathrm{~m}, 1 \mathrm{H}), 1.08(\mathrm{~s}, 9 \mathrm{H}) \mathrm{ppm} ;{ }^{13} \mathrm{C} \mathrm{NMR}\left(101 \mathrm{MHz}, \mathrm{CDCl}_{3}\right): \delta=194.59,151.35,141.69$, $135.87,133.89,133.81,130.00,129.97,127.85,127.81,67.84,31.94,27.02,21.28,19.27,18.89$ ppm; HRMS $(m / z):[\mathrm{M}+\mathrm{Na}]^{+}$calcd for $\mathrm{C}_{23} \mathrm{H}_{28} \mathrm{O}_{2} \mathrm{SiNa}^{+} 387.1751$, found 387.1755 . 


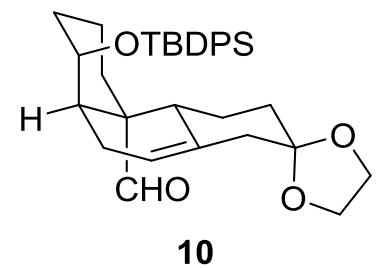

Tricyclic aldehyde 10: To a stirred solution of $\alpha, \beta$-unsaturated enal 12 (1.20 g, 3.29 mmol, 1.0 equiv) and diene $\mathbf{1 1}^{4}$ (1.10 g, $6.58 \mathrm{mmol}, 2.0$ equiv) in $\mathrm{CH}_{2} \mathrm{Cl}_{2}(10 \mathrm{~mL})$ was added $\mathrm{BF}_{3} \cdot \mathrm{OEt}_{2}(460 \mathrm{mg}, 400 \mu \mathrm{L}$, $3.24 \mathrm{mmol}, 1.0$ equiv) at $-78{ }^{\circ} \mathrm{C}$. The mixture was allowed to stir at that temperature for $50 \mathrm{~h}$ before saturated aq. $\mathrm{NaHCO}_{3}(50 \mathrm{~mL})$ was added. The resultant mixture was extracted with EtOAc $(3 \times 100 \mathrm{~mL})$. The combined organic phases were washed with brine $(50 \mathrm{~mL})$, dried over anhydrous $\mathrm{Na}_{2} \mathrm{SO}_{4}$, filtered, and concentrated under vacuum. The residue was purified by flash column chromatography with $\mathrm{Et}_{2} \mathrm{O} / \mathrm{CH}_{2} \mathrm{Cl}_{2} /$ petroleum ether $(1: 50: 50 \rightarrow 3: 50: 50)$ to give tricyclic aldehyde $\mathbf{1 0}(1.19 \mathrm{~g}, 68 \%)$ as a white foam. 10: $R_{\mathrm{f}}=0.33$ (silica, $\mathrm{Et}_{2} \mathrm{O} / \mathrm{CH}_{2} \mathrm{Cl}_{2} /$ petroleum ether 3:50:50); $[\alpha]_{\mathrm{D}}^{25}=-109.7(c=1.0$ in $\mathrm{CHCl}_{3}$ ); IR (film): $v_{\max }=3069,3047,2930,2856,2698,1722,1427,1375,1261,1106,868,702 \mathrm{~cm}^{-1}$; ${ }^{1} \mathrm{H}$ NMR $\left(400 \mathrm{MHz}, \mathrm{CDCl}_{3}\right): \delta=9.19(\mathrm{~s}, 1 \mathrm{H}), 7.70-7.63(\mathrm{~m}, 4 \mathrm{H}), 7.44-7.34(\mathrm{~m}, 6 \mathrm{H}), 5.50(\mathrm{~d}, J=4.7$ Hz, $1 \mathrm{H}), 4.06-3.89(\mathrm{~m}, 4 \mathrm{H}), 3.87-3.76(\mathrm{~m}, 1 \mathrm{H}), 2.53-2.40$ (m, $1 \mathrm{H}), 2.40-2.14(\mathrm{~m}, 4 \mathrm{H}), 1.88-1.78$ (m, $1 \mathrm{H}), 1.78-1.69(\mathrm{~m}, 1 \mathrm{H}), 1.61-1.51(\mathrm{~m}, 3 \mathrm{H}), 1.49-1.32(\mathrm{~m}, 4 \mathrm{H}), 1.33-1.24(\mathrm{~m}, 1 \mathrm{H}), 1.05(\mathrm{~s}, 9 \mathrm{H})$, $1.02-0.92(\mathrm{~m}, 1 \mathrm{H}) \mathrm{ppm} ;{ }^{13} \mathrm{C}$ NMR $\left(101 \mathrm{MHz}\right.$, acetone- $\left.\mathrm{d}_{6}\right): \delta=207.74,136.50,136.43,135.32,134.72$, $134.68,130.60,130.47,128.50,128.37,119.60,109.21,71.59,64.84,64.75,52.67,46.22,45.50,36.17$, $35.61,29.50,27.78,27.47,27.42,21.68,21.34,19.78 \mathrm{ppm}$; HRMS $(\mathrm{m} / \mathrm{z}):[\mathrm{M}+\mathrm{Na}]^{+}$calcd for $\mathrm{C}_{33} \mathrm{H}_{42} \mathrm{O}_{4} \mathrm{SiNa}^{+}$553.2745, found 553.2746.

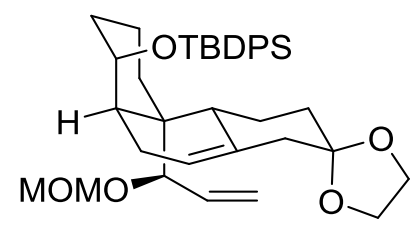

15

Allylic ether 15: To a stirred solution of tricyclic aldehyde 10 (12.6 g, $23.7 \mathrm{mmol}, 1.0$ equiv) in THF $(240 \mathrm{~mL})$ was added vinyllithium ${ }^{5}\left(60.0 \mathrm{~mL}, 0.45 \mathrm{M}\right.$ in THF, $27.0 \mathrm{mmol}, 1.1$ equiv) at $-78{ }^{\circ} \mathrm{C}$. The mixture was allowed to stir at that temperature for 30 min before a solution of $\mathrm{MOMCl}(2.97 \mathrm{~g}, 2.80 \mathrm{~mL}$, 
$36.9 \mathrm{mmol}, 1.6$ equiv) in THF ( $25 \mathrm{~mL})$ was added. The resultant mixture was allowed to warm to $22{ }^{\circ} \mathrm{C}$ and stir at that temperature for $30 \mathrm{~min}$. Saturated aq. $\mathrm{NaHCO}_{3}(150 \mathrm{~mL})$ was then added. The organic layer was separated, and the aqueous layer was extracted with EtOAc $(3 \times 300 \mathrm{~mL})$. The combined organic phases were washed with brine $(200 \mathrm{~mL})$, dried over anhydrous $\mathrm{Na}_{2} \mathrm{SO}_{4}$, filtered, and concentrated under vacuum. The residue was subjected to flash column chromatography for purification using EtOAc/petroleum ether $(1: 15 \rightarrow 1: 10)$ to give allylic ether $\mathbf{1 5}(12.2 \mathrm{~g}, 85 \%)$ as a white foam. 15: $R_{\mathrm{f}}=0.33$ (silica, EtOAc:petroleum ether 1:8); $[\alpha]_{\mathrm{D}}^{20}=-80.5\left(c=1.0\right.$ in $\left.\mathrm{CHCl}_{3}\right)$; IR (film): $v_{\max }=3070$, $2929,1471,1425,1260,1104,1032,924,876,702 \mathrm{~cm}^{-1} ;{ }^{1} \mathrm{H}$ NMR $\left(400 \mathrm{MHz}, \mathrm{CDCl}_{3}\right): \delta=7.71-7.62$ (m, 4 H), 7.46-7.32 (m, 6 H), $5.86(\mathrm{ddd}, J=17.2,10.2,9.2 \mathrm{~Hz}, 1 \mathrm{H}), 5.50-5.37(\mathrm{~m}, 1 \mathrm{H}), 5.24$ (dd, $J=$ 10.2, $1.1 \mathrm{~Hz}, 1 \mathrm{H}), 5.13(\mathrm{dd}, J=17.2,1.1 \mathrm{~Hz}, 1 \mathrm{H}), 4.34(\mathrm{~d}, J=6.3 \mathrm{~Hz}, 1 \mathrm{H}), 4.22(\mathrm{~d}, J=6.3 \mathrm{~Hz}, 1 \mathrm{H})$, 4.20-4.12 (m, 1 H), 4.09-3.90 (m, 5 H), 3.10 (s, 3 H), 2.65 (br s, 1 H), 2.53-2.38 (m, 1 H), 2.32 (dd, $J=$ 12.5, $2.5 \mathrm{~Hz}, 1 \mathrm{H}), 2.29-2.13(\mathrm{~m}, 3 \mathrm{H}), 1.87$ (dd, $J=13.1,2.5 \mathrm{~Hz}, 1 \mathrm{H}), 1.70-1.54(\mathrm{~m}, 1 \mathrm{H}), 1.58(\mathrm{dd}, J$ $=13.1,3.8 \mathrm{~Hz}, 1 \mathrm{H}), 1.53-1.24(\mathrm{~m}, 6 \mathrm{H}), 1.03(\mathrm{~s}, 9 \mathrm{H}), 0.98-0.89(\mathrm{~m}, 1 \mathrm{H}) \mathrm{ppm} ;{ }^{13} \mathrm{C} \mathrm{NMR}(101 \mathrm{MHz}$, $\left.\mathrm{CDCl}_{3}\right): \delta=135.93,135.39,135.29,135.17,134.55,129.53,129.49,127.53,127.47,119.71,118.50$, $109.62,95.80,81.02,69.47,64.56,64.41,55.98,46.40,42.67,36.33,33.65,28.92,28.19,27.25,27.05$, 22.37, 19.45, $19.34 \mathrm{ppm}$; HRMS $(\mathrm{m} / z):[\mathrm{M}+\mathrm{Na}]^{+}$calcd for $\mathrm{C}_{37} \mathrm{H}_{50} \mathrm{O}_{5} \mathrm{SiNa}^{+} 625.3320$, found 625.3325 .

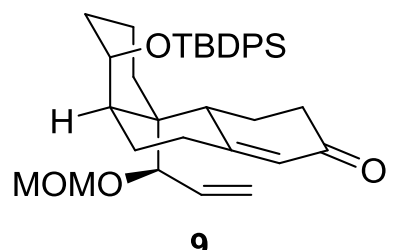

$\boldsymbol{\alpha}, \boldsymbol{\beta}$-Unsaturated enone 9: To a stirred solution of allylic ether $\mathbf{1 5}(4.65 \mathrm{~g}, 7.71 \mathrm{mmol}, 1.0$ equiv) in THF/acetone $(60 \mathrm{~mL}, 5: 1)$ was added aq. $\mathrm{HClO}_{4}(20.0 \mathrm{~mL}, 3.0 \mathrm{M})$ at $22{ }^{\circ} \mathrm{C}$. The mixture was allowed to stir at that temperature for $1.5 \mathrm{~h}$ before saturated aq. $\mathrm{NaHCO}_{3}(100 \mathrm{~mL})$ was added. The resultant mixture was extracted with EtOAc $(3 \times 200 \mathrm{~mL})$. The combined organic phases were washed with brine $(100 \mathrm{~mL})$, dried over anhydrous $\mathrm{Na}_{2} \mathrm{SO}_{4}$, filtered, and concentrated under vacuum. The residue was subjected to flash column chromatography for purification using EtOAc/petroleum ether $(1: 10 \rightarrow 1: 5)$ as eluent to give $\alpha, \beta$-unsaturated enone $9(3.10 \mathrm{~g}, 72 \%)$ as a white foam. 9: $R_{\mathrm{f}}=0.36$ (silica, 
EtOAc:petroleum ether 1:3); $[\alpha]_{\mathrm{D}}^{20}=-40.5\left(c=1.0\right.$ in $\left.\mathrm{CHCl}_{3}\right)$; IR (film): $v_{\max }=3071,3047,2932,2888$, 2822, 1669, 1609, 1471, 1426, 1256, 1105, 996, $756 \mathrm{~cm}^{-1} ;{ }^{1} \mathrm{H}$ NMR $\left(400 \mathrm{MHz}, \mathrm{CDCl}_{3}\right): \delta=7.71-7.60$ (m, 4 H), 7.47-7.39 (m, 2 H), 7.38-7.32 (m, 4 H), 5.95 (s, 1 H), 5.63 (ddd, J = 17.2, 10.3, 8.5 Hz, 1 H), $5.23(\mathrm{dd}, J=10.3,1.4 \mathrm{~Hz}, 1 \mathrm{H}), 5.16(\mathrm{~d}, J=17.2 \mathrm{~Hz}, 1 \mathrm{H}), 4.53(\mathrm{~d}, J=6.6 \mathrm{~Hz}, 1 \mathrm{H}), 4.33(\mathrm{~d}, J=6.6 \mathrm{~Hz}$, $1 \mathrm{H}), 4.30-4.23(\mathrm{~m}, 1 \mathrm{H}), 3.90(\mathrm{~d}, J=8.5 \mathrm{~Hz}, 1 \mathrm{H}), 3.72-3.53(\mathrm{~m}, 1 \mathrm{H}), 3.24(\mathrm{~s}, 3 \mathrm{H}), 3.02-2.87(\mathrm{~m}, 1$ H), 2.56-2.45 (m, $1 \mathrm{H}), 2.41-2.20(\mathrm{~m}, 3 \mathrm{H}), 2.17-2.07(\mathrm{~m}, 1 \mathrm{H}), 2.00-1.85(\mathrm{~m}, 2 \mathrm{H}), 1.84-1.70(\mathrm{~m}, 1$ $\mathrm{H}), 1.69-1.55(\mathrm{~m}, 2 \mathrm{H}), 1.46-1.22(\mathrm{~m}, 4 \mathrm{H}), 1.05(\mathrm{~s}, 9 \mathrm{H}) \mathrm{ppm} ;{ }^{13} \mathrm{C} \mathrm{NMR}\left(101 \mathrm{MHz}, \mathrm{CDCl}_{3}\right): \delta=$ $199.75,169.24,136.00,135.85,134.52,133.93,133.22,129.75,129.68,127.57,127.54,126.42,119.95$, $93.82,81.68,74.36,56.71,43.66,41.01,40.36,38.07,31.88,31.68,27.34,26.85,22.49,22.12,19.46$, 15.84 ppm; HRMS $(\mathrm{m} / z):[\mathrm{M}+\mathrm{Na}]^{+}$calcd for $\mathrm{C}_{35} \mathrm{H}_{46} \mathrm{O}_{4} \mathrm{SiNa}^{+}$581.3058, found 581.3060.

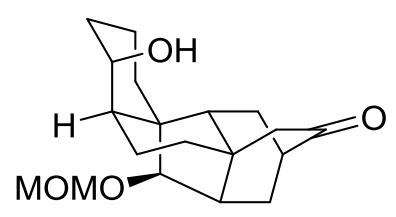

16

Cycloadduct 16: A solution of $\alpha, \beta$-unsaturated enone 9 (9.10 g, $16.3 \mathrm{mmol}, 1.0$ equiv) in THF (160 mL) was subjected to freeze-pump-thaw cycling for three times. To this solution was added LiHMDS (50.0 $\mathrm{mL}, 1.0 \mathrm{M}$ in THF, $50.0 \mathrm{mmol}, 3.1$ equiv) at $22^{\circ} \mathrm{C}$. The mixture was allowed to stir at that temperature for $7 \mathrm{~h}$ before TBAF ( $80.0 \mathrm{~mL}, 1.0 \mathrm{M}$ in THF, $80.0 \mathrm{mmol}, 4.9$ equiv) was added. The resultant mixture was heated to $70{ }^{\circ} \mathrm{C}$ and allowed to stir at that temperature for $5 \mathrm{~h}$. The mixture was cooled to $22{ }^{\circ} \mathrm{C}$, and saturated aq. $\mathrm{NH}_{4} \mathrm{Cl}(200 \mathrm{~mL})$ was added. The organic layer was separated, and the aqueous layer was extracted with EtOAc $(3 \times 300 \mathrm{~mL})$. The combined organic phases were washed with brine $(200 \mathrm{~mL})$, dried over anhydrous $\mathrm{Na}_{2} \mathrm{SO}_{4}$, and filtered. The volatiles were removed under vacuum, and the residue was purified by flash column chromatography with EtOAc/petroleum ether $(1: 5 \rightarrow 1: 2)$ to give cycloadduct $16(4.38 \mathrm{~g}, 84 \%)$ as a white solid. 16: $R_{\mathrm{f}}=0.30$ (silica, EtOAc:petroleum ether 1:2); $[\alpha]_{\mathrm{D}}^{20}=$ $-52.0\left(c=1.0\right.$ in $\left.\mathrm{CHCl}_{3}\right)$; IR (film): $v_{\max }=3485,2927,1714,1461,1393,1334,1238,1123,1042,922$, $757 \mathrm{~cm}^{-1} ;{ }^{1} \mathrm{H}$ NMR $\left(500 \mathrm{MHz}, \mathrm{CDCl}_{3}\right): \delta=4.73(\mathrm{~d}, J=6.6 \mathrm{~Hz}, 1 \mathrm{H}), 4.63(\mathrm{~d}, J=6.6 \mathrm{~Hz}, 1 \mathrm{H}), 4.08(\mathrm{~s}$, $1 \mathrm{H}), 3.46(\mathrm{~s}, 1 \mathrm{H}), 3.38(\mathrm{~s}, 3 \mathrm{H}), 2.70-2.62(\mathrm{~m}, 1 \mathrm{H}), 2.30(\mathrm{~d}, J=19.6 \mathrm{~Hz}, 1 \mathrm{H}), 2.23-2.19(\mathrm{~m}, 1 \mathrm{H})$, 
2.18-2.08 (m, $1 \mathrm{H}), 2.12(\mathrm{~d}, J=19.6 \mathrm{~Hz}, 1 \mathrm{H}), 2.08-2.01(\mathrm{~m}, 1 \mathrm{H}), 1.94-1.89(\mathrm{~m}, 1 \mathrm{H}), 1.89-1.82$ (m, 3 H), 1.82-1.78 (m, 1 H), 1.78-1.70 (m, $2 \mathrm{H}), 1.70-1.62(\mathrm{~m}, 1 \mathrm{H}), 1.61-1.52(\mathrm{~m}, 1 \mathrm{H}), 1.52-1.41$ (m, 3 H), 1.34-1.23 (m, $2 \mathrm{H}) \mathrm{ppm} ;{ }^{13} \mathrm{C}$ NMR (126 MHz, $\left.\mathrm{CDCl}_{3}\right): \delta=216.7,96.9,95.1,74.6,55.5,46.7,44.7$, 44.1, 43.2, 42.0, 40.8, 39.2, 35.3, 35.1, 34.2, 31.9, 25.4, 24.9, 18.4 ppm; $\operatorname{HRMS~}(\mathrm{m} / z):[\mathrm{M}+\mathrm{Na}]^{+} \mathrm{calcd}$ for $\mathrm{C}_{19} \mathrm{H}_{28} \mathrm{O}_{4} \mathrm{Na}^{+}$343.1880, found 343.1884. CCDC 1914278 contains the supplementary crystallographic data of $\mathbf{1 6}$ [m.p.: 112-115 ${ }^{\circ} \mathrm{C}$ (EtOAc:petroleum ether 1:10)]. These data can be obtained free of charge from The Cambridge Crystallographic Data Centre via www.ccdc.cam.ac.uk/data_request/cif.

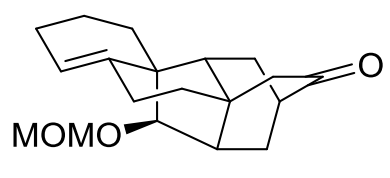

8

Pentacyclic intermediate 8: To a stirred solution of cycloadduct 16 (3.80 g, 11.9 mmol, 1.0 equiv) in $\mathrm{CH}_{2} \mathrm{Cl}_{2}(400 \mathrm{~mL})$ were sequentially added pyridine $\left(48.9 \mathrm{~g}, 50.0 \mathrm{~mL}, 618 \mathrm{mmol}, 52.0\right.$ equiv) and $\mathrm{SOCl}_{2}$ (7.22 g, $4.40 \mathrm{~mL}, 60.6 \mathrm{mmol}, 5.1$ equiv) at $0{ }^{\circ} \mathrm{C}$. The mixture was allowed to stir at that temperature for 1 h before saturated aq. $\mathrm{NaHCO}_{3}(200 \mathrm{~mL})$ was added. The organic layer was separated, and the aqueous layer was extracted with $\mathrm{CH}_{2} \mathrm{Cl}_{2}(3 \times 300 \mathrm{~mL})$. The combined organic phases were sequentially washed with aq. citric acid $(4 \times 200 \mathrm{~mL}, 10 \mathrm{wt} \%)$ and brine $(200 \mathrm{~mL})$, dried over anhydrous $\mathrm{Na}_{2} \mathrm{SO}_{4}$, filtered, and concentrated under vacuum. The residue was subjected to flash column chromatography for purification using EtOAc/petroleum ether $(1: 10 \rightarrow 1: 5)$ as eluent to give pentacyclic intermediate 8 $(2.73 \mathrm{~g}, 76 \%)$ as a white foam. 8: $R_{\mathrm{f}}=0.31$ (silica, EtOAc:petroleum ether $\left.1: 4\right) ;[\alpha]_{\mathrm{D}}^{20}=-156.9(c=1.0$ in $\mathrm{CHCl}_{3}$ ); IR (film): $v_{\max }=2927,1730,1453,1398,1327,1288,1128,1105,1030,960,861,799 \mathrm{~cm}^{-1}$; ${ }^{1} \mathrm{H}$ NMR $\left(500 \mathrm{MHz}, \mathrm{CDCl}_{3}\right): \delta=5.57-5.51(\mathrm{~m}, 1 \mathrm{H}), 4.76(\mathrm{~d}, J=6.8 \mathrm{~Hz}, 1 \mathrm{H}), 4.66(\mathrm{~d}, J=6.8 \mathrm{~Hz}, 1 \mathrm{H})$, 3.63-3.58 (m, $1 \mathrm{H}), 3.39(\mathrm{~s}, 3 \mathrm{H}), 2.78-2.68(\mathrm{~m}, 1 \mathrm{H}), 2.33(\mathrm{~d}, J=19.6 \mathrm{~Hz}, 1 \mathrm{H}), 2.29-2.24(\mathrm{~m}, 1 \mathrm{H})$, $2.12(\mathrm{~d}, J=19.6 \mathrm{~Hz}, 1 \mathrm{H}), 2.15-2.02(\mathrm{~m}, 2 \mathrm{H}), 2.02-1.91(\mathrm{~m}, 5 \mathrm{H}), 1.90-1.84(\mathrm{~m}, 1 \mathrm{H}), 1.84-1.75$ (m, 2 $\mathrm{H}), 1.70-1.60(\mathrm{~m}, 3 \mathrm{H}), 1.54-1.44(\mathrm{~m}, 2 \mathrm{H}) \mathrm{ppm} ;{ }^{13} \mathrm{C} \mathrm{NMR}\left(126 \mathrm{MHz}, \mathrm{CDCl}_{3}\right): \delta=215.6,140.8,121.6$, 
97.1, 95.9, 55.7, 48.8, 48.1, 44.4, 44.2, 44.1, 41.9, 37.9, 34.3, 30.6, 28.9, 25.7, 25.5, 20.9 ppm; HRMS

$(\mathrm{m} / z):[\mathrm{M}+\mathrm{Na}]^{+}$calcd for $\mathrm{C}_{19} \mathrm{H}_{26} \mathrm{O}_{3} \mathrm{Na}^{+} 325.1774$, found 325.1779 .

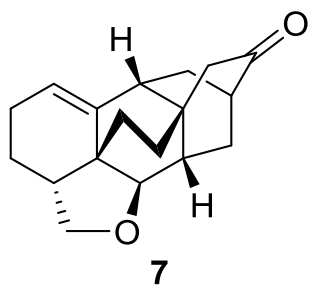

Hexacyclic intermediate 7: To a stirred solution of pentacyclic intermediate $8(2.57 \mathrm{~g}, 8.50 \mathrm{mmol}, 1.0$ equiv) in $\mathrm{CH}_{2} \mathrm{Cl}_{2}(100 \mathrm{~mL})$ was added $\mathrm{SnCl}_{4}\left(9.00 \mathrm{~mL}, 1.0 \mathrm{M}\right.$ in $\mathrm{CH}_{2} \mathrm{Cl}_{2}, 9.00 \mathrm{mmol}, 1.1$ equiv) at $-15^{\circ} \mathrm{C}$. The mixture was allowed to stir at that temperature for $1 \mathrm{~h}$ before saturated aq. $\mathrm{NH}_{4} \mathrm{Cl}(100 \mathrm{~mL})$ was added. The resultant mixture was extracted with EtOAc $(3 \times 200 \mathrm{~mL})$, and the combined organic phases were washed with brine $(100 \mathrm{~mL})$, dried over anhydrous $\mathrm{Na}_{2} \mathrm{SO}_{4}$, and filtered. The volatiles were removed under vacuum, and the residue was purified by flash column chromatography with EtOAc/petroleum ether $(1: 10 \rightarrow 1: 5)$ to give hexacyclic intermediate $7(1.45 \mathrm{~g}, 63 \%)$ as a white foam. 7 : $R_{\mathrm{f}}=0.33$ (silica, EtOAc:petroleum ether 1:3); $[\alpha]_{\mathrm{D}}^{20}=-69.2\left(c=1.0\right.$ in $\left.\mathrm{CHCl}_{3}\right) ; \mathrm{IR}\left(\right.$ film): $v_{\max }=2930$, $2858,1729,1460,1400,1259,1153,1043,960,806 \mathrm{~cm}^{-1} ;{ }^{1} \mathrm{H}$ NMR $\left(400 \mathrm{MHz}, \mathrm{CDCl}_{3}\right): \delta=5.59-5.51$ (m, $1 \mathrm{H}), 4.30(\mathrm{dd}, J=9.1,7.8 \mathrm{~Hz}, 1 \mathrm{H}), 3.61-3.51(\mathrm{~m}, 1 \mathrm{H}), 3.44(\mathrm{dd}, J=9.1,4.4 \mathrm{~Hz}, 1 \mathrm{H}), 2.38-2.11(\mathrm{~m}$, $4 \mathrm{H}), 2.20(\mathrm{~d}, J=18.8 \mathrm{~Hz}, 1 \mathrm{H}), 2.01(\mathrm{~d}, J=18.8 \mathrm{~Hz}, 1 \mathrm{H}), 1.97-1.91(\mathrm{~m}, 2 \mathrm{H}), 1.89-1.79(\mathrm{~m}, 3 \mathrm{H})$, 1.79-1.67 (m, $2 \mathrm{H}), 1.57-1.45$ (m, $2 \mathrm{H}), 1.42-1.31$ (m, $1 \mathrm{H}), 1.31-1.18$ (m, $1 \mathrm{H}), 1.18-1.05$ (m, $1 \mathrm{H}) \mathrm{ppm}$; ${ }^{13} \mathrm{C}$ NMR $\left(126 \mathrm{MHz}, \mathrm{CDCl}_{3}\right): \delta=216.1,146.3,119.8,89.9,74.9,47.2,45.8,43.8,40.1,39.7,37.6,36.4$, 34.9, 32.9, 30.9, 28.1, 26.6, 24.3 ppm; HRMS $(m / z):[\mathrm{M}+\mathrm{Na}]^{+}$calcd for $\mathrm{C}_{18} \mathrm{H}_{22} \mathrm{O}_{2} \mathrm{Na}^{+}$293.1512, found 293.1519.

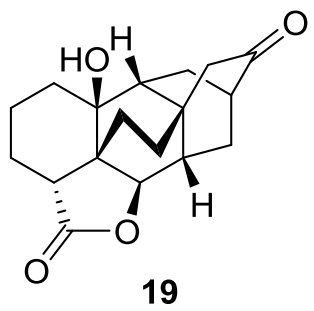

Hexacyclic lactone 19: To a stirred solution of hexacyclic intermediate 7 (2.26 g, $8.36 \mathrm{mmol}, 1.0$ equiv) and $\mathrm{PhSiH}_{3}\left(8.77 \mathrm{~g}, 10.0 \mathrm{~mL}, 81.1 \mathrm{mmol}, 9.7\right.$ equiv) in $\mathrm{EtOH}(70 \mathrm{~mL})$ was added $\mathrm{Mn}(\mathrm{acac})_{2}(212 \mathrm{mg}$, 
$0.837 \mathrm{mmol}, 10 \mathrm{~mol} \%$ ) at $22{ }^{\circ} \mathrm{C}$. Oxygen was then bubbled through the mixture for $30 \mathrm{~min}$. The resultant mixture was allowed to stir under an oxygen atmosphere at $22{ }^{\circ} \mathrm{C}$ for $2 \mathrm{~h}$. The volatiles were removed under vacuum, and the residue was passed through a short plug of silica gel with EtOAc/petroleum ether $(1: 4 \rightarrow 1: 1)$ to give the tertiary alcohol $(1.95 \mathrm{~g}, 81 \%)$ as a pale yellow oil. This oil was dissolved in MeCN/EtOAc (40 mL, 1:1). To the stirred solution were sequentially added phosphate buffer $(30 \mathrm{~mL}, 1.0 \mathrm{M}, \mathrm{pH}=7.0), \mathrm{NaIO}_{4}\left(7.23 \mathrm{~g}, 33.8 \mathrm{mmol}, 5.0\right.$ equiv), and $\mathrm{RuCl}_{3} \cdot 3 \mathrm{H}_{2} \mathrm{O}$ (88.4 mg, $0.338 \mathrm{mmol}, 5.0 \mathrm{~mol} \%$ ) at $22{ }^{\circ} \mathrm{C}$. The mixture was allowed to stir at that temperature for $24 \mathrm{~h}$ before saturated aq. $\mathrm{NaHCO}_{3}(50 \mathrm{~mL})$ and saturated aq. $\mathrm{Na}_{2} \mathrm{~S}_{2} \mathrm{O}_{3}(150 \mathrm{~mL})$ were sequentially added. The resultant mixture was extracted with $\mathrm{CH}_{2} \mathrm{Cl}_{2}(3 \times 200 \mathrm{~mL})$. The combined organic phases were washed with brine $(100 \mathrm{~mL})$, dried over anhydrous $\mathrm{Na}_{2} \mathrm{SO}_{4}$, filtered, and concentrated under vacuum. The residue was subjected to flash column chromatography for purification using EtOAc/petroleum ether $(1: 4 \rightarrow 1: 1)$ as eluent to give hexacyclic lactone $19(1.43 \mathrm{~g}, 70 \%)$ as a white powder. 19: $R_{\mathrm{f}}=0.34$ (silica, EtOAc:petroleum ether 1:2); $[\alpha]_{\mathrm{D}}^{25}=-82.7\left(c=1.0\right.$ in $\mathrm{CHCl}_{3}$ ); IR (film): $v_{\max }=3473,2928$, 2867, 1747, 1727, 1456, 1330, 1261, 1092, 1019, $798 \mathrm{~cm}^{-1} ;{ }^{1} \mathrm{H}$ NMR $\left(400 \mathrm{MHz}, \mathrm{CDCl}_{3}\right): \delta=4.32(\mathrm{dd}$, $J=5.7,1.3 \mathrm{~Hz}, 1 \mathrm{H}), 2.38-2.34(\mathrm{~m}, 1 \mathrm{H}), 2.34-2.18(\mathrm{~m}, 2 \mathrm{H}), 2.26(\mathrm{~d}, J=19.3 \mathrm{~Hz}, 1 \mathrm{H}), 2.06(\mathrm{~d}, J=$ $19.3 \mathrm{~Hz}, 1 \mathrm{H}), 2.11-1.92(\mathrm{~m}, 4 \mathrm{H}), 1.89-1.81(\mathrm{~m}, 2 \mathrm{H}), 1.80-1.67(\mathrm{~m}, 3 \mathrm{H}), 1.67-1.53(\mathrm{~m}, 3 \mathrm{H})$, $1.53-1.43$ (m, $3 \mathrm{H}), 1.43-1.31(\mathrm{~m}, 1 \mathrm{H}) \mathrm{ppm} ;{ }^{13} \mathrm{C} \mathrm{NMR}\left(126 \mathrm{MHz}, \mathrm{CDCl}_{3}\right): \delta=214.9,179.1,86.1,72.3$, 49.0, 47.0, 45.4, 44.9, 42.8, 36.9, 34.8, 32.2, 31.7, 29.1, 27.2, 23.6, 22.7, $19.2 \mathrm{ppm} ; \mathrm{HRMS}(\mathrm{m} / \mathrm{z}):[\mathrm{M}+$ $\mathrm{Na}^{+}$calcd for $\mathrm{C}_{18} \mathrm{H}_{22} \mathrm{O}_{4} \mathrm{Na}^{+}$325.1410, found 325.1412. CCDC 1914280 contains the supplementary crystallographic data of 19 [m.p.: 262-264 ${ }^{\circ} \mathrm{C}\left(\mathrm{CHCl}_{3}\right.$ :petroleum ether 1:5)]. These data can be obtained free of charge from The Cambridge Crystallographic Data Centre via www.ccdc.cam.ac.uk/data_request/cif. 


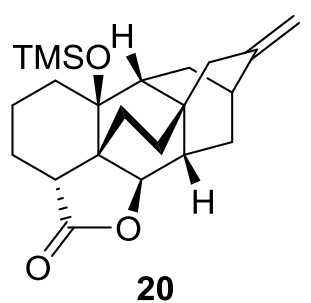

Silyl ether 20: To a stirred suspension of methyltriphenylphosphonium bromide ( $2.90 \mathrm{~g}, 8.12 \mathrm{mmol}, 2.1$ equiv) in THF $(20 \mathrm{~mL})$ was added $t$-BuOK $\left(8.00 \mathrm{~mL}, 1.0 \mathrm{M}\right.$ in THF, $8.00 \mathrm{mmol}, 2.1$ equiv) at $22{ }^{\circ} \mathrm{C}$. The resultant mixture was allowed to stir at that temperature for $15 \mathrm{~min}$. A solution of hexacyclic lactone $19(1.17 \mathrm{~g}, 3.87 \mathrm{mmol}, 1.0$ equiv) in THF $(10 \mathrm{~mL})$ was then added, and the mixture was allowed to stir at $22{ }^{\circ} \mathrm{C}$ for $30 \mathrm{~min}$. To the resultant mixture were sequentially added $\mathrm{Et}_{3} \mathrm{~N}(3.92 \mathrm{~g}, 5.40 \mathrm{~mL}, 38.7$ mmol, 10 equiv) and TMSOTf ( $6.89 \mathrm{~g}, 5.60 \mathrm{~mL}, 31.0 \mathrm{mmol}, 8.0$ equiv) at $0{ }^{\circ} \mathrm{C}$. The resultant mixture was allowed to stir at that temperature for 15 min before saturated aq. $\mathrm{NaHCO}_{3}(100 \mathrm{~mL})$ was added. The mixture was extracted with EtOAc $(3 \times 200 \mathrm{~mL})$, and the combined organic phases were washed with brine $(100 \mathrm{~mL})$, dried over anhydrous $\mathrm{Na}_{2} \mathrm{SO}_{4}$, and filtered. The volatiles were removed under vacuum, and the residue was purified by flash column chromatography with EtOAc/petroleum ether $(1: 30 \rightarrow 1: 20)$ to give silyl ether $\mathbf{2 0}(1.23 \mathrm{~g}, 85 \%)$ as a white foam. $\mathbf{2 0}: \boldsymbol{R}_{\mathrm{f}}=0.31$ (silica, EtOAc:petroleum ether 1:10); $[\alpha]_{\mathrm{D}}^{25}=-82.4\left(c=1.0\right.$ in $\left.\mathrm{CHCl}_{3}\right)$; IR (film): $v_{\max }=3070,2982,2946$, $2867,1773,1454,1260,1249,1137,1025,835,753 \mathrm{~cm}^{-1} ;{ }^{1} \mathrm{H}$ NMR $\left(500 \mathrm{MHz}, \mathrm{CDCl}_{3}\right): \delta=4.75-4.70$ $(\mathrm{m}, 1 \mathrm{H}), 4.61-4.56(\mathrm{~m}, 1 \mathrm{H}), 4.22(\mathrm{~d}, J=5.6 \mathrm{~Hz}, 1 \mathrm{H}), 2.32-2.27(\mathrm{~m}, 1 \mathrm{H}), 2.25-2.15(\mathrm{~m}, 2 \mathrm{H})$, 2.08-2.02 (s, $1 \mathrm{H}), 2.02-1.95(\mathrm{~m}, 2 \mathrm{H}), 1.94-1.85(\mathrm{~m}, 1 \mathrm{H}), 1.84-1.70(\mathrm{~m}, 2 \mathrm{H}), 1.69-1.63(\mathrm{~m}, 3 \mathrm{H})$, 1.63-1.54 (m, $4 \mathrm{H}), 1.54-1.49$ (m, $1 \mathrm{H}), 1.39-1.28$ (m, $3 \mathrm{H}), 0.14$ (s, $9 \mathrm{H}) \mathrm{ppm} ;{ }^{13} \mathrm{C}$ NMR (126 MHz, $\left.\mathrm{CDCl}_{3}\right): \delta=179.8,150.6,104.8,87.0,76.2,48.3,46.9,45.3,39.3,36.4,36.1,34.9,34.6,31.3,30.9$, 29.6, 23.9, 23.5, 19.6, $2.6 \mathrm{ppm}$; HRMS $(\mathrm{m} / \mathrm{z}):[\mathrm{M}+\mathrm{H}]^{+}$calcd for $\mathrm{C}_{22} \mathrm{H}_{33} \mathrm{O}_{3} \mathrm{Si}^{+}$373.2193, found 373.2195 . 


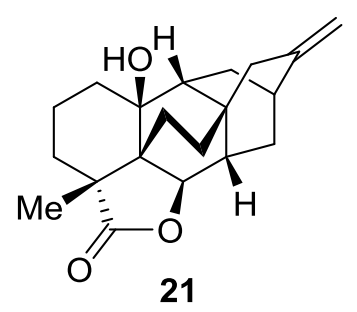

Methylated lactone 21: To a stirred solution of $i-\mathrm{Pr}_{2} \mathrm{NH}(1.52 \mathrm{~g}, 2.10 \mathrm{~mL}, 15.0 \mathrm{mmol}, 3.1$ equiv) in THF $(20 \mathrm{~mL})$ was added $\mathrm{BuLi}\left(6.80 \mathrm{~mL}, 2.13 \mathrm{M}\right.$ in hexane, $14.5 \mathrm{mmol}, 3.0$ equiv) at $0{ }^{\circ} \mathrm{C}$. The mixture was allowed to stir at that temperature for $30 \mathrm{~min}$ before HMPA $(10.3 \mathrm{~g}, 10.0 \mathrm{~mL}, 57.5 \mathrm{mmol})$ and a solution of silyl ether $\mathbf{2 0}$ (1.78 g, $4.78 \mathrm{mmol}, 1.0$ equiv) in THF (10 mL) were sequentially added. The resultant mixture was allowed to stir at $0{ }^{\circ} \mathrm{C}$ for $1 \mathrm{~h}$ before MeOTf $(2.50 \mathrm{~g}, 1.70 \mathrm{~mL}, 15.0 \mathrm{mmol}, 3.1$ equiv) was added. The mixture was allowed to stir at that temperature for $1 \mathrm{~h}$ before TBAF $(50.0 \mathrm{~mL}$, $1.0 \mathrm{M}$ in THF, $50.0 \mathrm{mmol}, 10$ equiv) was added. The resultant mixture was allowed to warm to $22{ }^{\circ} \mathrm{C}$ and stir at that temperature for $3 \mathrm{~h}$. Saturated aq. $\mathrm{NaHCO}_{3}(100 \mathrm{~mL})$ was then added, and the mixture was extracted with EtOAc $(3 \times 200 \mathrm{~mL})$. The combined organic phases were washed with brine $(100$ $\mathrm{mL}$ ), dried over anhydrous $\mathrm{Na}_{2} \mathrm{SO}_{4}$, filtered, and concentrated under vacuum. The residue was subjected to flash column chromatography for purification using EtOAc/petroleum ether $(1: 10 \rightarrow 1: 5)$ as eluent to give methylated lactone $21(1.10 \mathrm{~g}, 73 \%)$ as a white powder. 21: $R_{\mathrm{f}}=0.38$ (silica, EtOAc:petroleum ether 1:3); $[\alpha]_{\mathrm{D}}^{25}=-100.6\left(c=1.0\right.$ in $\left.\mathrm{CHCl}_{3}\right)$; IR (film): $v_{\max }=3504,3066,2930,2864,1753,1655$, 1463, 1379, 1260, 1100, 923, 872, $667 \mathrm{~cm}^{-1} ;{ }^{1} \mathrm{H}$ NMR (500 MHz, $\left.\mathrm{CDCl}_{3}\right): \delta=4.74-4.69(\mathrm{~m}, 1 \mathrm{H})$, 4.60-4.54 (m, $1 \mathrm{H}), 4.18(\mathrm{dd}, J=5.8,1.2 \mathrm{~Hz}, 1 \mathrm{H}), 2.34-2.27(\mathrm{~m}, 1 \mathrm{H}), 2.24-2.16(\mathrm{~m}, 1 \mathrm{H}), 2.14-2.06$ (m, 2 H), 2.05-1.98 (m, $1 \mathrm{H}), 1.91-1.77$ (m, $2 \mathrm{H}), 1.76-1.68$ (m, 2 H), 1.68-1.55 (m, $6 \mathrm{H}), 1.51-1.43$ $(\mathrm{m}, 1 \mathrm{H}), 1.44-1.34(\mathrm{~m}, 2 \mathrm{H}), 1.20(\mathrm{~s}, 3 \mathrm{H}), 1.27-1.13(\mathrm{~m}, 2 \mathrm{H}) \mathrm{ppm} ;{ }^{13} \mathrm{C}$ NMR $\left(126 \mathrm{MHz}, \mathrm{CDCl}_{3}\right): \delta=$ 182.1, 150.6, 104.7, 85.4, 73.6, 50.3, 48.3, 45.0, 39.0, 36.5, 35.9, 34.6, 34.1, 32.6, 31.9, 31.4, 29.7, 19.1, 18.7, 17.0 ppm; HRMS (m/z): $[\mathrm{M}+\mathrm{H}]^{+}$calcd for $\mathrm{C}_{20} \mathrm{H}_{27} \mathrm{O}_{3}{ }^{+} 315.1955$, found 315.1959. 


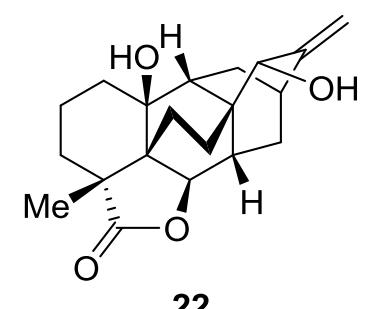

Allylic alcohol 22: To a stirred solution of methylated lactone 21 (1.45 g, $4.61 \mathrm{mmol}, 1.0$ equiv) in $\mathrm{CH}_{2} \mathrm{Cl}_{2}(40 \mathrm{~mL})$ were sequentially added $\mathrm{SeO}_{2}(5.11 \mathrm{~g}, 4.60 \mathrm{mmol}, 1.0$ equiv) and TBHP (4.00 mL, $5.5 \mathrm{M}$ in decane, $22.0 \mathrm{mmol}, 4.8$ equiv) at $0{ }^{\circ} \mathrm{C}$. The mixture was allowed to stir at that temperature for $3 \mathrm{~h}$ before saturated aq. $\mathrm{Na}_{2} \mathrm{SO}_{3}(100 \mathrm{~mL})$ was added. The resultant mixture was extracted with EtOAc $(3 \times$ $100 \mathrm{~mL}$ ), and the combined organic phases were washed with brine $(50 \mathrm{~mL})$, dried over anhydrous $\mathrm{Na}_{2} \mathrm{SO}_{4}$, and filtered. The volatiles were removed under vacuum, and the residue was purified by flash column chromatography with EtOAc/petroleum ether $(1: 2 \rightarrow 1: 1)$ to give allylic alcohol $22(1.23 \mathrm{~g}, 81 \%)$ as a white foam. 22: $R_{\mathrm{f}}=0.37$ (silica, EtOAc:petroleum ether 1:1); $[\alpha]_{\mathrm{D}}^{25}=-89.8(c=1.0$ in acetone); IR (film): $v_{\max }=3401,2970,2943,2864,1759,1689,1445,1302,1047,1029,991,730 \mathrm{~cm}^{-1} ;{ }^{1} \mathrm{H} \mathrm{NMR}$ $\left(400 \mathrm{MHz}, \mathrm{CDCl}_{3}\right): \delta=5.01-4.98(\mathrm{~m}, 1 \mathrm{H}), 4.98-4.96(\mathrm{~m}, 1 \mathrm{H}), 4.23(\mathrm{dd}, J=5.7,1.5 \mathrm{~Hz}, 1 \mathrm{H}), 3.83(\mathrm{br} \mathrm{s}$, $1 \mathrm{H}), 2.44-2.37(\mathrm{~m}, 1 \mathrm{H}), 2.17-2.02(\mathrm{~m}, 3 \mathrm{H}), 2.02-1.93(\mathrm{~m}, 1 \mathrm{H}), 1.93-1.83(\mathrm{~m}, 1 \mathrm{H}), 1.80-1.67(\mathrm{~m}, 2 \mathrm{H})$, 1.67-1.56 (m, 5 H), 1.56-1.39 (m, $3 \mathrm{H}), 1.34-1.14$ (m, $3 \mathrm{H}), 1.21$ (s, $3 \mathrm{H}) \mathrm{ppm} ;{ }^{13} \mathrm{C}$ NMR $(126 \mathrm{MHz}$, acetone- $\left.\mathrm{d}_{6}\right): \delta=182.0,156.2,109.0,85.8,73.7,73.3,49.2,48.2,45.5,38.9,36.7,34.3,32.8,32.4,31.8$, 31.5, 25.9, 19.8, 19.1, 17.7 ppm; HRMS ( $m / z):[\mathrm{M}+\mathrm{H}]^{+}$calcd for $\mathrm{C}_{20} \mathrm{H}_{27} \mathrm{O}_{4}{ }^{+} 331.1904$, found 331.1911.

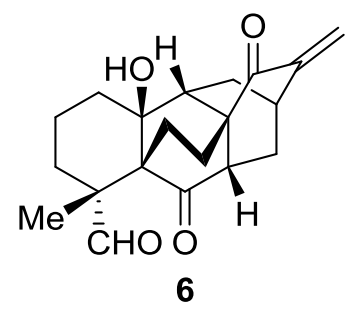

Diketoaldehyde 6: To a stirred solution of allylic alcohol $22(1.21 \mathrm{~g}, 3.66 \mathrm{mmol}, 1.0$ equiv) in THF (35 $\mathrm{mL})$ was added $\mathrm{LiAlH}_{4}\left(417 \mathrm{mg}, 11.0 \mathrm{mmol}, 3.0\right.$ equiv) at $22{ }^{\circ} \mathrm{C}$. The mixture was heated to $70{ }^{\circ} \mathrm{C}$ and allowed to stir at that temperature for $4 \mathrm{~h}$ before it was cooled to $22{ }^{\circ} \mathrm{C}$. Saturated aq. $\mathrm{NH}_{4} \mathrm{Cl}(100 \mathrm{~mL})$ was then added, and the resultant mixture was extracted with $\mathrm{CH}_{2} \mathrm{Cl}_{2}(3 \times 150 \mathrm{~mL})$. The combined 
organic phases were washed with brine $(100 \mathrm{~mL})$, dried over anhydrous $\mathrm{Na}_{2} \mathrm{SO}_{4}$, and filtered. The volatiles were removed under vacuum, and the residue (crude tetraol) was dissolved in $\mathrm{CH}_{2} \mathrm{Cl}_{2}(35 \mathrm{~mL}$ ). To a stirred solution of pyridine $\left(7.24 \mathrm{~g}, 7.40 \mathrm{~mL}, 91.5 \mathrm{mmol}, 25\right.$ equiv) in $\mathrm{CH}_{2} \mathrm{Cl}_{2}(20 \mathrm{~mL})$ were sequentially added Celite (13.2 g) and $\mathrm{CrO}_{3}\left(4.39 \mathrm{~g}, 43.9 \mathrm{mmol}, 12\right.$ equiv) at $22{ }^{\circ} \mathrm{C}$. ${ }^{6}$ The mixture was allowed to stir at that temperature for $15 \mathrm{~min}$ before the solution of crude tetraol was added. The resultant mixture was allowed to stir at $22{ }^{\circ} \mathrm{C}$ for $20 \mathrm{~min}$ and then filtered through a short plug of Celite. The filter cake was washed with $\mathrm{CH}_{2} \mathrm{Cl}_{2}(3 \times 100 \mathrm{~mL})$, and the filtrate was washed with brine $(100 \mathrm{~mL})$ and dried over anhydrous $\mathrm{Na}_{2} \mathrm{SO}_{4}$. After filtration and removal of the volatiles under vacuum, the residue was subjected to flash column chromatography for purification using EtOAc/petroleum ether $(1: 4 \rightarrow 1: 2)$ as eluent to give diketoaldehyde $\mathbf{6}(696 \mathrm{mg}, 58 \%$ for the two steps $)$ as a white foam. $6: R_{\mathrm{f}}=$ 0.29 (silica, EtOAc:petroleum ether 1:2); $[\alpha]_{\mathrm{D}}^{25}=-50.3\left(c=1.0\right.$ in $\left.\mathrm{CHCl}_{3}\right)$; IR (film): $v_{\max }=3482,2959$, 2923, 2853, 1701, 1626, 1465, 1378, 1261, 1096, 1018, $797 \mathrm{~cm}^{-1} ;{ }^{1} \mathrm{H} \mathrm{NMR}\left(400 \mathrm{MHz}, \mathrm{CDCl}_{3}\right): \delta=$ $9.55(\mathrm{~s}, 1 \mathrm{H}), 5.96(\mathrm{~d}, J=1.3 \mathrm{~Hz}, 1 \mathrm{H}), 5.20(\mathrm{~d}, J=1.3 \mathrm{~Hz}, 1 \mathrm{H}), 2.77-2.70(\mathrm{~m}, 1 \mathrm{H}), 2.51-2.42$ (m, 1 H), 2.34-2.25 (m, 1 H), 2.23-2.14 (m, $1 \mathrm{H}), 2.14-1.99(\mathrm{~m}, 4 \mathrm{H}), 1.99-1.83(\mathrm{~m}, 4 \mathrm{H}), 1.83-1.70$ (m, 3 $\mathrm{H}), 1.70-1.48(\mathrm{~m}, 4 \mathrm{H}), 1.21(\mathrm{~s}, 3 \mathrm{H}) \mathrm{ppm} ;{ }^{13} \mathrm{C}$ NMR $\left(126 \mathrm{MHz}, \mathrm{CDCl}_{3}\right): \delta=217.0,205.6,200.6,146.7$, 117.0, 77.7, 58.3, 48.5, 47.6, 46.5, 44.5, 36.1, 35.1, 32.2, 30.8, 28.4, 21.2, 20.0, 19.5, 16.5 ppm; HRMS $(m / z):[\mathrm{M}+\mathrm{Na}]^{+}$calcd for $\mathrm{C}_{20} \mathrm{H}_{24} \mathrm{O}_{4} \mathrm{Na}^{+} 351.1567$, found 351.1565 .

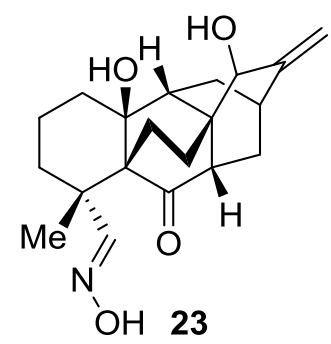

Oxime 23: To a stirred solution of diketoaldehyde 6 (319 mg, $0.971 \mathrm{mmol}, 1.0$ equiv) in $\mathrm{MeOH}(10 \mathrm{~mL})$ were sequentially added $\mathrm{NaOAc}\left(95.6 \mathrm{mg}, 1.17 \mathrm{mmol}, 1.2\right.$ equiv) and $\mathrm{NH}_{2} \mathrm{OH} \cdot \mathrm{HCl}(68.8 \mathrm{mg}, 0.990$ mmol, 1.0 equiv) at $22{ }^{\circ} \mathrm{C}$. The mixture was allowed to stir at that temperature for $2 \mathrm{~h}$ and then concentrated under vacuum. The residue was dissolved in THF/MeOH (11 mL, 10:1). To the stirred solution was added $\mathrm{NaBH}_{4}\left(73.3 \mathrm{mg}, 1.94 \mathrm{mmol}, 2.0\right.$ equiv) at $-78{ }^{\circ} \mathrm{C}$. The mixture was allowed to stir 
at that temperature for $15 \mathrm{~min}$ before saturated aq. $\mathrm{NaHCO}_{3}(50 \mathrm{~mL})$ was added. The resultant mixture was extracted with EtOAc $(3 \times 100 \mathrm{~mL})$. The combined organic phases were washed with brine $(50$ $\mathrm{mL}$ ), dried over anhydrous $\mathrm{Na}_{2} \mathrm{SO}_{4}$, and filtered. The volatiles were removed under vacuum, and the residue was purified by flash column chromatography with $\mathrm{MeOH} / \mathrm{CH}_{2} \mathrm{Cl}_{2}(1: 30 \rightarrow 1: 15)$ to give oxime $23(218 \mathrm{mg}, 65 \%)$ as a white foam. 23: $R_{\mathrm{f}}=0.32$ (silica, $\left.\mathrm{MeOH}: \mathrm{CH}_{2} \mathrm{Cl}_{2} 1: 15\right) ;[\alpha]_{\mathrm{D}}^{25}=-21.6(c=1.0$ in $\mathrm{MeOH}$ ); IR (film): $v_{\max }=3447,2960,2925,2870,1702,1647,1461,1261,1097,1036,799 \mathrm{~cm}^{-1} ;{ }^{1} \mathrm{H}$ NMR (400 MHz, methanol-d 4 ): $\delta=7.52(\mathrm{~s}, 1 \mathrm{H})$, 5.04-4.99 (m, $1 \mathrm{H}), 4.94-4.89(\mathrm{~m}, 1 \mathrm{H}), 3.98-3.93(\mathrm{~m}$, $1 \mathrm{H}), 2.40-2.24(\mathrm{~m}, 2 \mathrm{H}), 2.24-2.14(\mathrm{~m}, 2 \mathrm{H}), 2.08-1.97$ (m, $2 \mathrm{H}), 1.99-1.80(\mathrm{~m}, 2 \mathrm{H}), 1.74-1.57$ (m, 4 $\mathrm{H}), 1.54-1.37$ (m, $5 \mathrm{H}), 1.34(\mathrm{~s}, 3 \mathrm{H}) \mathrm{ppm} ;{ }^{13} \mathrm{C}$ NMR (126 MHz, methanol-d 4$): \delta=220.2,160.8,155.8$, $107.7,78.2,74.0,58.8,47.8,44.9,41.4,40.5,37.2,36.4,35.4,31.1,30.1,26.1,23.9,20.0,18.0$ ppm; $\operatorname{HRMS}(\mathrm{m} / z):[\mathrm{M}+\mathrm{H}]^{+}$calcd for $\mathrm{C}_{20} \mathrm{H}_{28} \mathrm{NO}_{4}{ }^{+}$346.2013, found 346.2016.

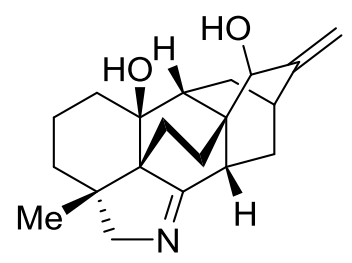

3: arcutinidine

Arcutinidine (3): To a stirred solution of oxime 23 (197 mg, $0.570 \mathrm{mmol}, 1.0$ equiv) in $\mathrm{MeOH}(5.0 \mathrm{~mL})$ were sequentially added $\mathrm{NH}_{4} \mathrm{OAc}$ ( $879 \mathrm{mg}, 11.4$ mmol, 20 equiv), $\mathrm{NaBH}_{3} \mathrm{CN}$ (107 mg, 1.70 mmol, 3.0 equiv), and aq. $\mathrm{TiCl}_{3}\{1.30 \mathrm{~mL}, 20 \% \mathrm{w} / \mathrm{v}$ [in aq. $\mathrm{HCl}(2.0 \mathrm{M})], 1.70 \mathrm{mmol}, 3.0$ equiv $\}$ at $0{ }^{\circ} \mathrm{C}$. The mixture was allowed to stir at that temperature for $1 \mathrm{~h}$, and aq. $\mathrm{NaOH}(1.0 \mathrm{M})$ was added to adjust the $\mathrm{pH}$ level to 10 . The resultant mixture was allowed to stir at $22{ }^{\circ} \mathrm{C}$ for $10 \mathrm{~min}$ and then extracted with EtOAc $(4 \times 30 \mathrm{~mL})$. The combined organic phases were washed with brine $(40 \mathrm{~mL})$, dried over anhydrous $\mathrm{Na}_{2} \mathrm{SO}_{4}$, filtered, and concentrated under vacuum. The residue was subjected to flash column chromatography for purification using $\mathrm{MeOH} / \mathrm{CH}_{2} \mathrm{Cl}_{2}(1: 30 \rightarrow 1: 15)$ as eluent to give arcutinidine (3; $127 \mathrm{mg}, 71 \%)$ as a white solid. 3: $R_{\mathrm{f}}=0.30$ (silica, $\left.\mathrm{MeOH}: \mathrm{CH}_{2} \mathrm{Cl}_{2} 1: 15\right) ;[\alpha]_{\mathrm{D}}^{25}=+3.9(c=1.0$ in $\mathrm{CHCl}_{3}$ ); IR (film): $v_{\max }=3422,3072,2930,2866,1648,1459,1377,1260,1053,972,770 \mathrm{~cm}^{-1} ;{ }^{1} \mathrm{H}$ NMR (500 MHz, $\left.\mathrm{CDCl}_{3}\right): \delta=5.08-5.05(\mathrm{~m}, 1 \mathrm{H}), 5.01-4.97(\mathrm{~m}, 1 \mathrm{H}), 3.98(\mathrm{~s}, 1 \mathrm{H}), 3.71(\mathrm{dd}, J=15.3$, 
$2.2 \mathrm{~Hz}, 1 \mathrm{H}), 3.45$ (dd, $J=15.3,2.2 \mathrm{~Hz}, 1 \mathrm{H}), 2.35-2.26(\mathrm{~m}, 2 \mathrm{H}), 2.12-2.03(\mathrm{~m}, 2 \mathrm{H}), 1.98-1.90$ (m, 2 H), 1.89-1.84 (m, $1 \mathrm{H}), 1.78-1.65(\mathrm{~m}, 4 \mathrm{H}), 1.64-1.58(\mathrm{~m}, 2 \mathrm{H}), 1.58-1.51(\mathrm{~m}, 2 \mathrm{H}), 1.44-1.30$ (m, 4 $\mathrm{H}), 0.95(\mathrm{~s}, 3 \mathrm{H}) \mathrm{ppm} ;{ }^{13} \mathrm{C}$ NMR $\left(126 \mathrm{MHz}, \mathrm{CDCl}_{3}\right): \delta=186.4,155.4,108.6,75.1,73.6,73.3,58.9$, 42.6, 41.0, 40.3, 37.2, 35.4, 32.7, 31.8, 30.7, 28.9, 26.1, 23.6, 20.2, 16.6 ppm; HRMS (m/z): $[\mathrm{M}+\mathrm{H}]^{+}$ calcd for $\mathrm{C}_{20} \mathrm{H}_{28} \mathrm{NO}_{2}^{+}$314.2115, found 314.2117. CCDC 1914281 contains the supplementary crystallographic data of 3 [m.p.: $159-161{ }^{\circ} \mathrm{C}$ (EtOAc:petroleum ether 1:5)]. These data can be obtained free of charge from The Cambridge Crystallographic Data Centre via Www.ccdc.cam.ac.uk/data_request/cif.

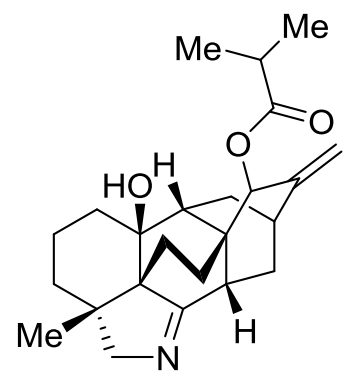

2: arcutinine

Arcutinine (2): To a stirred solution of $i-\mathrm{PrCO}_{2} \mathrm{H}(9.3 \mathrm{mg}, 0.11 \mathrm{mmol}, 1.5$ equiv) and 4-DMAP (8.6 mg, 0.070 mmol, 1.0 equiv) in $\mathrm{CH}_{2} \mathrm{Cl}_{2}(100 \mu \mathrm{L})$ was added DCC (100 $\mu \mathrm{L}, 1.0 \mathrm{M}$ in $\mathrm{CH}_{2} \mathrm{Cl}_{2}, 0.100$ mmol, 1.4 equiv) at $22{ }^{\circ} \mathrm{C}$. The mixture was allowed to stir at that temperature for $5 \mathrm{~min}$ before a solution of arcutinidine (3; $22.1 \mathrm{mg}, 0.0705 \mathrm{mmol}, 1.0$ equiv) in $\mathrm{CH}_{2} \mathrm{Cl}_{2}(600 \mu \mathrm{L})$ was added. The resultant mixture was allowed to stir at $22{ }^{\circ} \mathrm{C}$ for $3 \mathrm{~h}$. Saturated aq. $\mathrm{NaHCO}_{3}(10 \mathrm{~mL})$ was then added, and the mixture was extracted with EtOAc $(3 \times 20 \mathrm{~mL})$. The combined organic phases were washed with brine $(10 \mathrm{~mL})$, dried over anhydrous $\mathrm{Na}_{2} \mathrm{SO}_{4}$, filtered, and concentrated under vacuum. The residue was purified by flash column chromatography with acetone/ $\mathrm{CH}_{2} \mathrm{Cl}_{2}(1: 10 \rightarrow 1: 5)$ to give arcutinine $(2 ; 20.8 \mathrm{mg}, 77 \%)$ as a white solid. 2: $R_{\mathrm{f}}=0.26$ (silica, acetone: $\left.\mathrm{CH}_{2} \mathrm{Cl}_{2} 1: 10\right) ;[\alpha]_{\mathrm{D}}^{25}=-15.4\left(c=0.50\right.$ in $\left.\mathrm{CHCl}_{3}\right)$; IR (film): $v_{\max }=3218,2959,2926,2854,1734,1649,1457,1385,1189,1154,1097,1009,975,945 \mathrm{~cm}^{-1} ;{ }^{1} \mathrm{H}$ $\operatorname{NMR}\left(400 \mathrm{MHz}, \mathrm{CDCl}_{3}\right): \delta=5.43-5.35(\mathrm{~m}, 1 \mathrm{H}), 4.98-4.92(\mathrm{~m}, 1 \mathrm{H}), 4.90-4.83(\mathrm{~m}, 1 \mathrm{H}), 3.70(\mathrm{dd}, J=$ 15.3, 2.2 Hz, $1 \mathrm{H}), 3.45$ (dd, $J=15.3,2.2 \mathrm{~Hz}, 1 \mathrm{H}), 2.68-2.55$ (m, $1 \mathrm{H}), 2.48-2.38$ (m, $1 \mathrm{H}), 2.37-2.31$ (m, $1 \mathrm{H}), 2.06-1.95(\mathrm{~m}, 3 \mathrm{H}), 1.90-1.83$ (m, $1 \mathrm{H}), 1.81-1.71$ (m, $3 \mathrm{H}), 1.69-1.60$ (m, $2 \mathrm{H}), 1.60-1.51$ 
(m, $3 \mathrm{H}), 1.46-1.36(\mathrm{~m}, 2 \mathrm{H}), 1.36-1.24(\mathrm{~m}, 2 \mathrm{H}), 1.21(\mathrm{~d}, J=3.9 \mathrm{~Hz}, 3 \mathrm{H}), 1.19(\mathrm{~d}, J=3.9 \mathrm{~Hz}, 3 \mathrm{H})$,

0.95 (s, $3 \mathrm{H}) \mathrm{ppm} ;{ }^{13} \mathrm{C}$ NMR $\left(126 \mathrm{MHz}, \mathrm{CDCl}_{3}\right): \delta=185.5,177.1,150.4,110.2,75.0,73.7,73.4,58.7$, 43.6, 41.0, 39.3, 37.1, 35.4, 34.4, 33.1, 32.2, 30.8, 28.6, 25.9, 23.3, 20.2, 19.3, 19.2, 16.7 ppm; HRMS $(m / z):[\mathrm{M}+\mathrm{H}]^{+}$calcd for $\mathrm{C}_{24} \mathrm{H}_{34} \mathrm{NO}_{3}{ }^{+}$384.2533, found 384.2538. CCDC 1915624 contains the supplementary crystallographic data of 2 [m.p.: $245-247^{\circ} \mathrm{C}\left(\mathrm{CH}_{2} \mathrm{Cl}_{2}\right.$ :petroleum ether 1:10)]. These data can be obtained free of charge from The Cambridge Crystallographic Data Centre via www.ccdc.cam.ac.uk/data_request/cif.

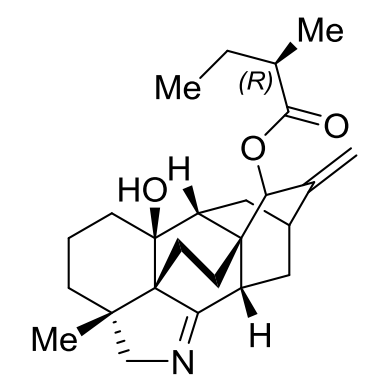

1: the originally described structure of arcutine

The originally described structure of $\operatorname{arcutine}^{7}(1)$ : To a stirred solution of $(R)-s-\mathrm{BuCO}_{2} \mathrm{H}(2.1 \mathrm{mg}$, $0.021 \mathrm{mmol}, 1.5$ equiv) and 4-DMAP (1.7 mg, $0.014 \mathrm{mmol}, 1.0$ equiv) in $\mathrm{CH}_{2} \mathrm{Cl}_{2}(50 \mu \mathrm{L})$ was added DCC $\left(20.0 \mu \mathrm{L}, 1.0 \mathrm{M}\right.$ in $\mathrm{CH}_{2} \mathrm{Cl}_{2}, 0.0200 \mathrm{mmol}, 1.4$ equiv) at $22{ }^{\circ} \mathrm{C}$. The mixture was allowed to stir at that temperature for $5 \mathrm{~min}$ before a solution of arcutinidine $(\mathbf{3} ; 4.4 \mathrm{mg}, 0.014 \mathrm{mmol}, 1.0$ equiv) in $\mathrm{CH}_{2} \mathrm{Cl}_{2}(150 \mu \mathrm{L})$ was added. The resultant mixture was allowed to stir at $22{ }^{\circ} \mathrm{C}$ for $3 \mathrm{~h}$. Saturated aq. $\mathrm{NaHCO}_{3}(5 \mathrm{~mL})$ was then added, and the mixture was extracted with EtOAc $(4 \times 5 \mathrm{~mL})$. The combined organic phases were washed with brine $(5 \mathrm{~mL})$, dried over anhydrous $\mathrm{Na}_{2} \mathrm{SO}_{4}$, filtered, and concentrated under vacuum. The residue was subjected to flash column chromatography for purification using acetone $/ \mathrm{CH}_{2} \mathrm{Cl}_{2}(1: 10 \rightarrow 1: 5)$ as eluent to give the originally described structure of arcutine ${ }^{7}(\mathbf{1} ; 4.1 \mathrm{mg}$, $74 \%$ ) as a white solid. 1: $R_{\mathrm{f}}=0.37$ (silica, acetone: $\left.\mathrm{CH}_{2} \mathrm{Cl}_{2} 1: 6\right) ;[\alpha]_{\mathrm{D}}^{25}=-16.3\left(c=0.34\right.$ in $\left.\mathrm{CHCl}_{3}\right) ; \mathrm{IR}^{2}$ (film): $v_{\max }=2961,2925,2855,1732,1461,1261,1091,1020,800 \mathrm{~cm}^{-1} ;{ }^{1} \mathrm{H} \mathrm{NMR}\left(400 \mathrm{MHz}, \mathrm{CDCl}_{3}\right)$ : $\delta=5.45-5.36(\mathrm{~m}, 1 \mathrm{H}), 5.00-4.92(\mathrm{~m}, 1 \mathrm{H}), 4.92-4.84(\mathrm{~m}, 1 \mathrm{H}), 3.70(\mathrm{dd}, J=15.3,2.3 \mathrm{~Hz}, 1 \mathrm{H}), 3.45$ (dd, $J=15.3,2.2 \mathrm{~Hz}, 1 \mathrm{H}), 2.50-2.37$ (m, $2 \mathrm{H}), 2.37-2.30(\mathrm{~m}, 1 \mathrm{H}), 2.08-1.93(\mathrm{~m}, 3 \mathrm{H}), 1.91-1.82$ (m, 
$1 \mathrm{H}), 1.82-1.69$ (m, 4 H), 1.68-1.60 (m, $2 \mathrm{H}), 1.59-1.45$ (m, $4 \mathrm{H}), 1.45-1.27$ (m, $4 \mathrm{H}), 1.18$ (d, $J=7.0$

$\mathrm{Hz}, 3 \mathrm{H}), 0.95$ (s, $3 \mathrm{H}), 0.93$ (t, $J=7.6 \mathrm{~Hz}, 3 \mathrm{H}) \mathrm{ppm} ;{ }^{13} \mathrm{C} \mathrm{NMR}\left(151 \mathrm{MHz}, \mathrm{CDCl}_{3}\right): \delta=185.4,176.7$, $150.5,110.2,75.1,73.7,73.5,58.7,43.6,41.7,41.0,39.2,37.1,35.4,33.1,32.2,30.9,28.6,26.8,26.0$, 23.3, 20.2, 17.0, 16.7, 11.9 ppm; HRMS $(m / z):[\mathrm{M}+\mathrm{H}]^{+}$calcd for $\mathrm{C}_{25} \mathrm{H}_{36} \mathrm{NO}_{3}{ }^{+}$398.2690, found 398.2690. CCDC 1938476 contains the supplementary crystallographic data of $\mathbf{1}$ [m.p.: $222-224{ }^{\circ} \mathrm{C}$ $\left(\mathrm{CH}_{2} \mathrm{Cl}_{2}\right.$ :hexane 1:1)]. These data can be obtained free of charge from The Cambridge Crystallographic Data Centre via www.ccdc.cam.ac.uk/data_request/cif. There are three alerts level B in the checkCIF report of these data, which are attributable to the difficulties of acquiring and processing massive data caused by three molecules contained in a single crystal cell. These alerts have no bearing on the structural elucidation.

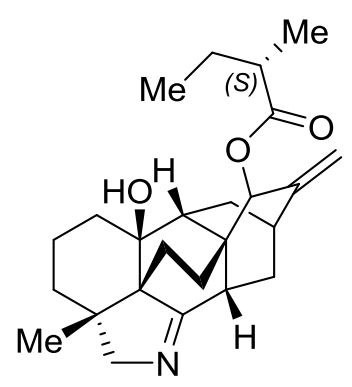

25: arcutine

Arcutine (25): To a stirred solution of $(S)-s-\mathrm{BuCO}_{2} \mathrm{H}(2.5 \mathrm{mg}, 0.025 \mathrm{mmol}, 1.5$ equiv) and 4-DMAP (1.9 mg, 0.016 mmol, 1.0 equiv) in $\mathrm{CH}_{2} \mathrm{Cl}_{2}(50 \mu \mathrm{L})$ was added DCC $\left(22.0 \mu \mathrm{L}, 1.0 \mathrm{M}\right.$ in $\mathrm{CH}_{2} \mathrm{Cl}_{2}, 0.0220$ mmol, 1.4 equiv) at $22{ }^{\circ} \mathrm{C}$. The mixture was allowed to stir at that temperature for 5 min before a solution of arcutinidine (3; $5.0 \mathrm{mg}, 0.016 \mathrm{mmol}, 1.0$ equiv) in $\mathrm{CH}_{2} \mathrm{Cl}_{2}(150 \mu \mathrm{L})$ was added. The resultant mixture was allowed to stir at $22{ }^{\circ} \mathrm{C}$ for $3 \mathrm{~h}$. Saturated aq. $\mathrm{NaHCO}_{3}(5 \mathrm{~mL})$ was then added, and the mixture was extracted with EtOAc $(4 \times 5 \mathrm{~mL})$. The combined organic phases were washed with brine $(5$ $\mathrm{mL}$ ), dried over anhydrous $\mathrm{Na}_{2} \mathrm{SO}_{4}$, and filtered. The volatiles were removed under vacuum, and the residue was purified by flash column chromatography with acetone/ $\mathrm{CH}_{2} \mathrm{Cl}_{2}(1: 10 \rightarrow 1: 5)$ to give arcutine $(\mathbf{2 5} ; 4.7 \mathrm{mg}, 74 \%)$ as a white solid. 25: $R_{\mathrm{f}}=0.37$ (silica, acetone: $\left.\mathrm{CH}_{2} \mathrm{Cl}_{2} 1: 6\right) ;[\alpha]_{\mathrm{D}}^{25}=+1.7(c=$ 0.80 in $\mathrm{CHCl}_{3}$ ); IR (film): $v_{\max }=3224,2962,2934,2862,1731,1649,1459,1261,1028,802 \mathrm{~cm}^{-1} ;{ }^{1} \mathrm{H}$ $\operatorname{NMR}\left(400 \mathrm{MHz}, \mathrm{CDCl}_{3}\right): \delta=5.48-5.33(\mathrm{~m}, 1 \mathrm{H}), 5.01-4.92(\mathrm{~m}, 1 \mathrm{H}), 4.92-4.84(\mathrm{~m}, 1 \mathrm{H}), 3.70(\mathrm{dd}, J=$ 
$15.3,1.8 \mathrm{~Hz}, 1 \mathrm{H}), 3.45(\mathrm{dd}, J=15.3,1.7 \mathrm{~Hz}, 1 \mathrm{H}), 2.51-2.37$ (m, $2 \mathrm{H}), 2.37-2.29$ (m, $1 \mathrm{H}), 2.08-1.93$ (m, 3 H), 1.91-1.82 (m, 1 H), 1.82-1.68 (m, 4 H), 1.68-1.60 (m, 2 H), 1.60-1.45 (m, 4 H), 1.45-1.26 $(\mathrm{m}, 4 \mathrm{H}), 1.18(\mathrm{~d}, J=7.0 \mathrm{~Hz}, 3 \mathrm{H}), 0.95(\mathrm{~s}, 3 \mathrm{H}), 0.93(\mathrm{t}, J=7.5 \mathrm{~Hz}, 3 \mathrm{H}) \mathrm{ppm} ;{ }^{13} \mathrm{C}$ NMR $(101 \mathrm{MHz}$, $\left.\mathrm{CDCl}_{3}\right): \delta=185.4,176.8,150.4,110.3,75.0,73.7,73.5,58.6,43.5,41.5,41.0,39.2,37.1,35.4,33.2$, $32.3,30.8,28.5,27.0,25.9,23.2,20.2,17.0,16.7,11.8 \mathrm{ppm}$; HRMS $(\mathrm{m} / \mathrm{z}):[\mathrm{M}+\mathrm{H}]^{+}$calcd for $\mathrm{C}_{25} \mathrm{H}_{36} \mathrm{NO}_{3}{ }^{+}$398.2690, found 398.2692. CCDC 1938477 contains the supplementary crystallographic data of 25 [m.p.: $220-222{ }^{\circ} \mathrm{C}\left(\mathrm{CH}_{2} \mathrm{Cl}_{2}\right.$ :hexane 1:1)]. These data can be obtained free of charge from The Cambridge Crystallographic Data Centre via www.ccdc.cam.ac.uk/data_request/cif. 
II HPLC Traces for Measuring Enantiomeric Excess<smiles>O[C@H]1C=C(Br)CCC1</smiles>

13

Racemic and enantioenriched 13 were analyzed by HPLC (CHIRALPAK IG column, $i$-PrOH:hexane $2: 98,0.80 \mathrm{~mL} / \mathrm{min}$ ) to determine the enantiomeric excess (ee). For 13, ee $=99.7 \%$.

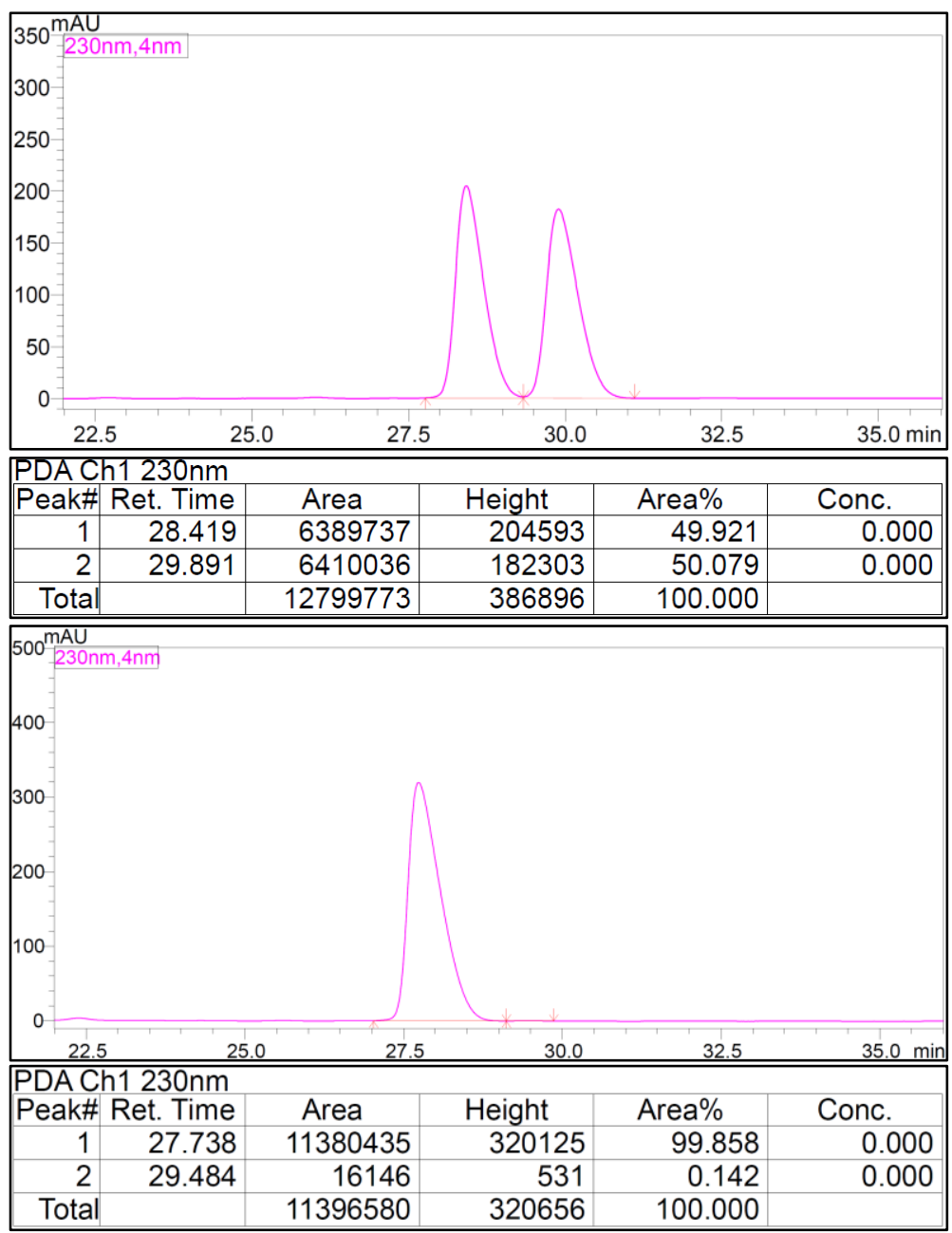


III $\quad{ }^{1} \mathrm{H}$ and ${ }^{13} \mathrm{C}$ NMR Spectra of Compounds

${ }^{1} \mathrm{H}$ NMR Spectrum of $14\left(500 \mathrm{MHz}, \mathrm{CDCl}_{3}\right)$

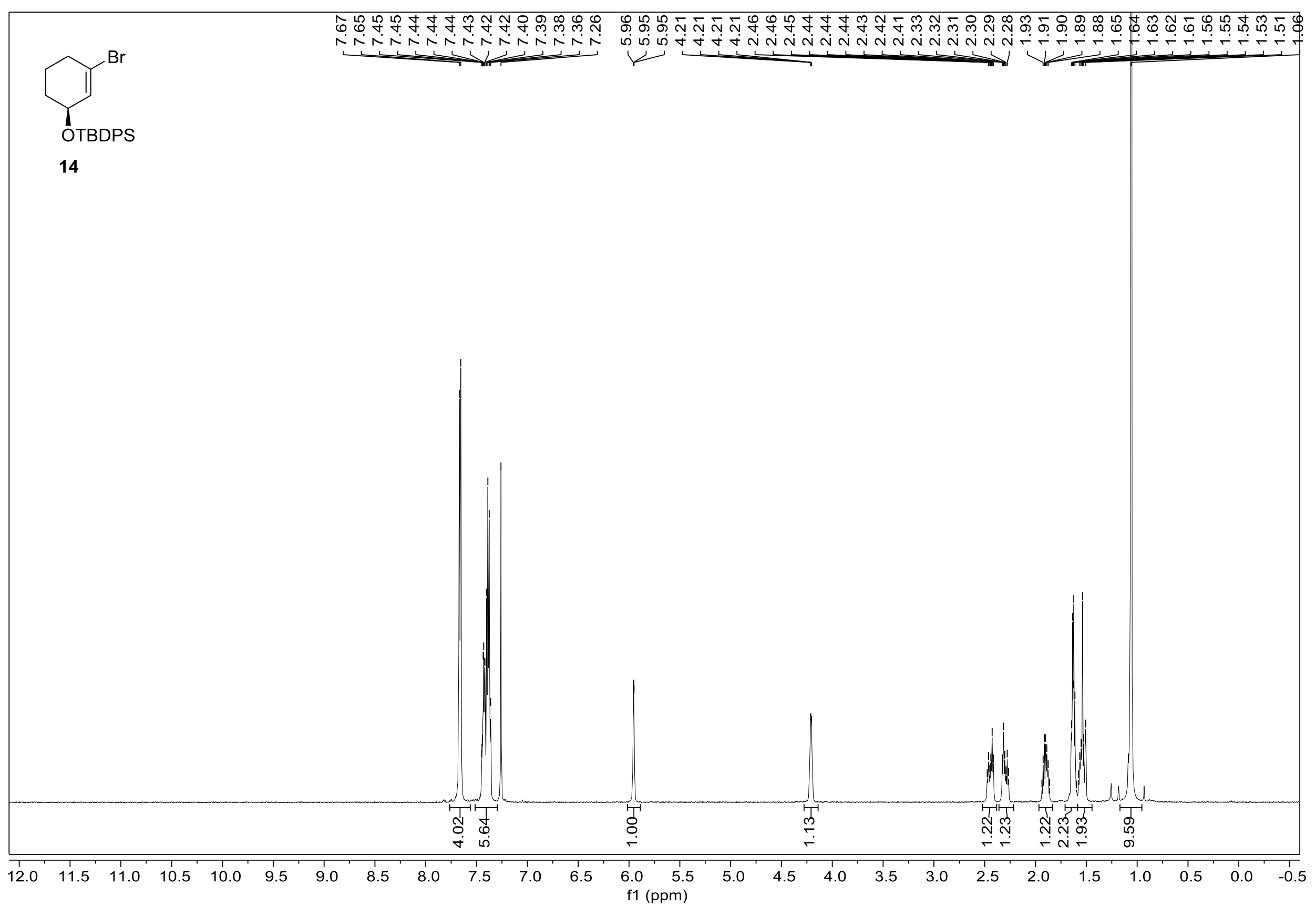


${ }^{13} \mathrm{C}$ NMR Spectrum of $14\left(101 \mathrm{MHz}, \mathrm{CDCl}_{3}\right)$

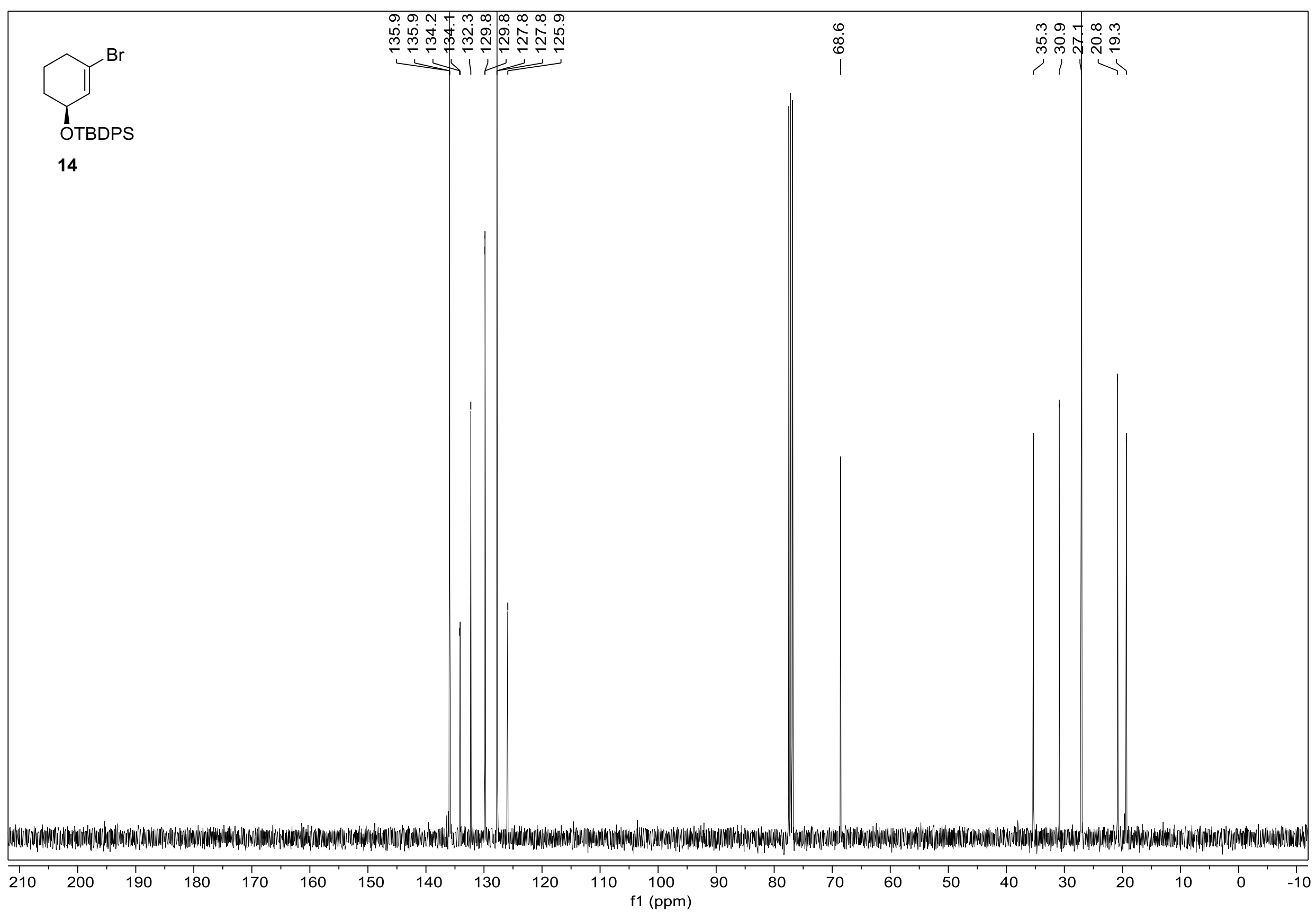


${ }^{1} \mathrm{H}$ NMR Spectrum of $12\left(400 \mathrm{MHz}, \mathrm{CDCl}_{3}\right)$

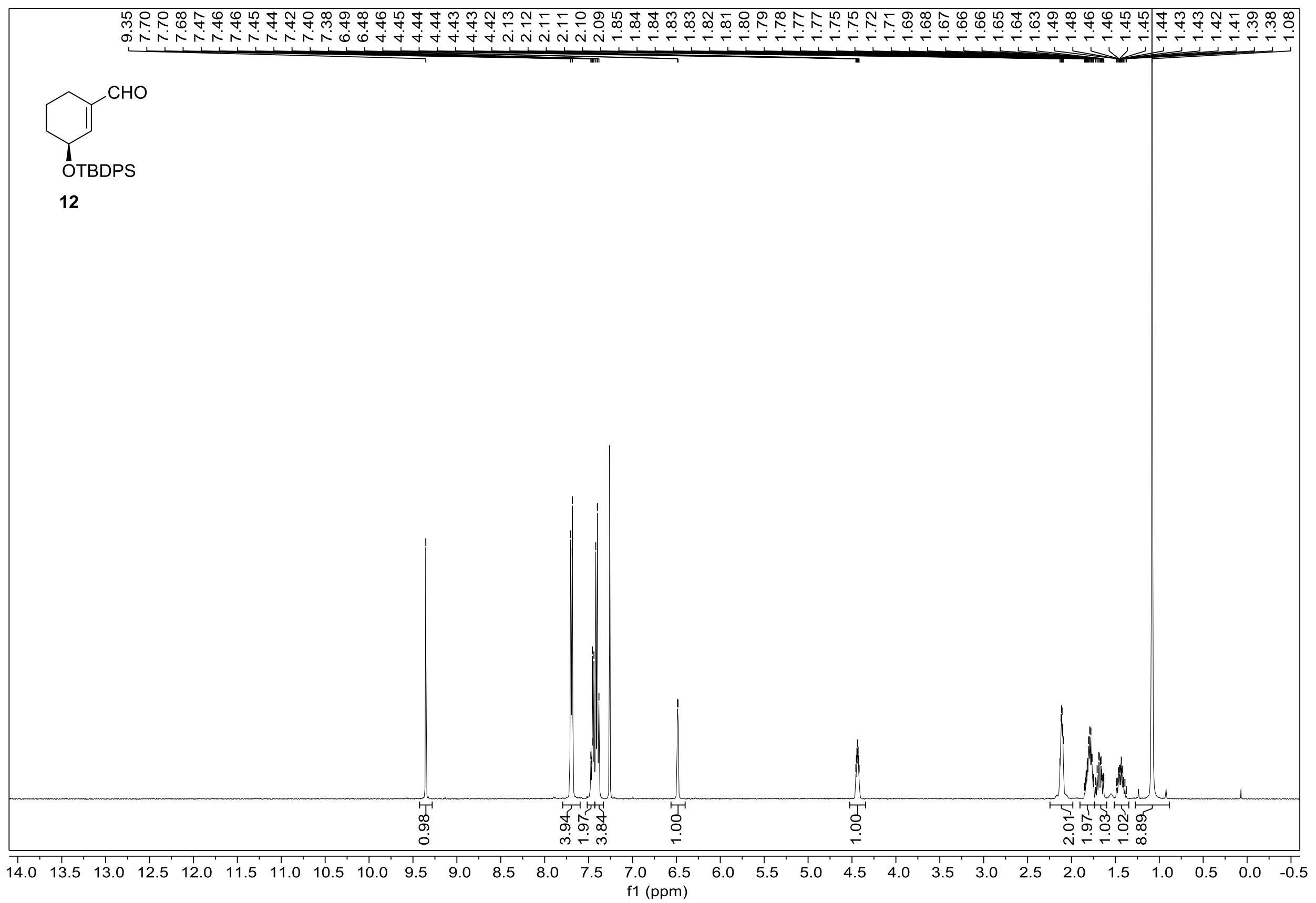


${ }^{13} \mathrm{C}$ NMR Spectrum of $12\left(101 \mathrm{MHz}, \mathrm{CDCl}_{3}\right)$

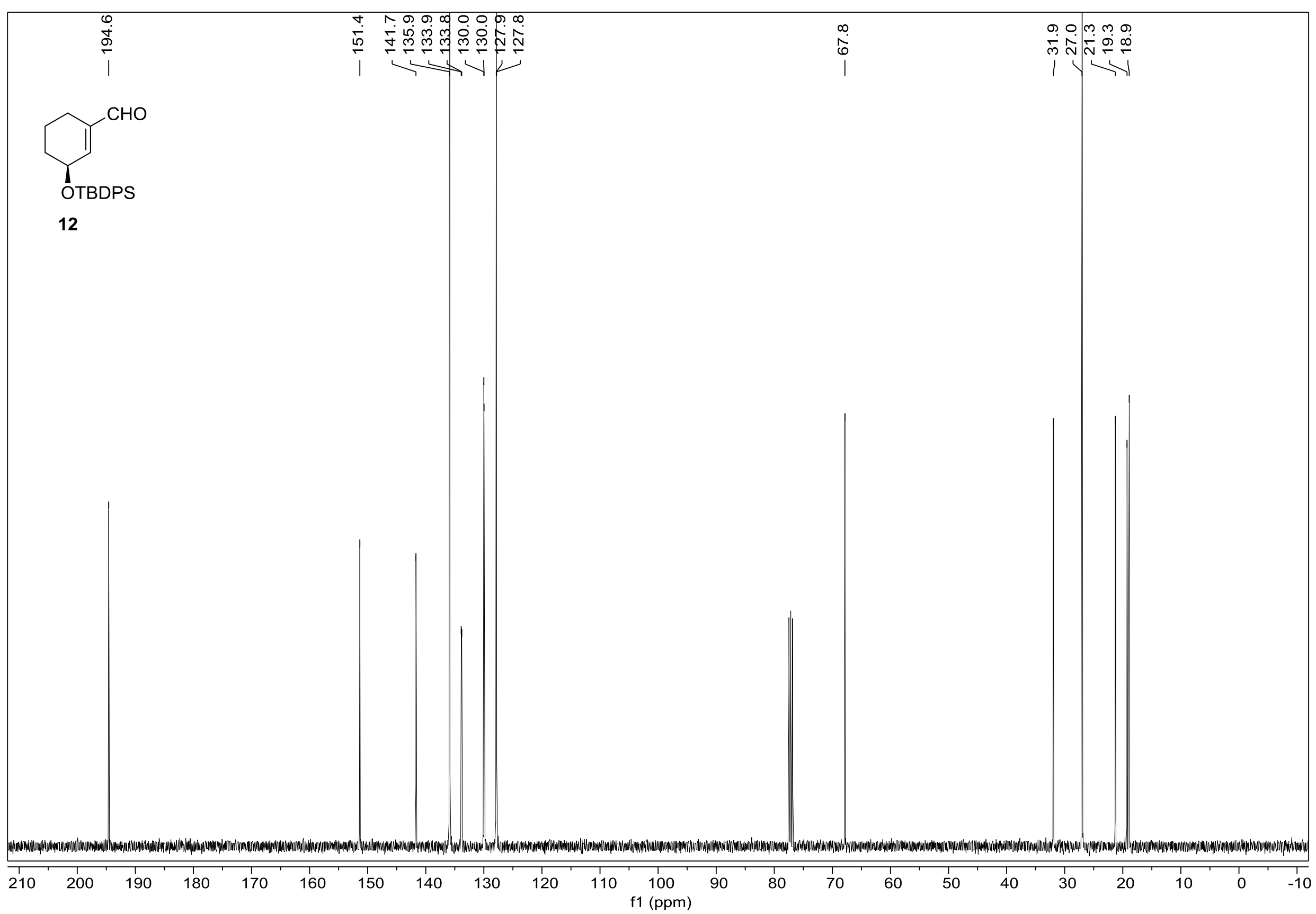


${ }^{1} \mathrm{H}$ NMR Spectrum of $10\left(400 \mathrm{MHz}, \mathrm{CDCl}_{3}\right)$

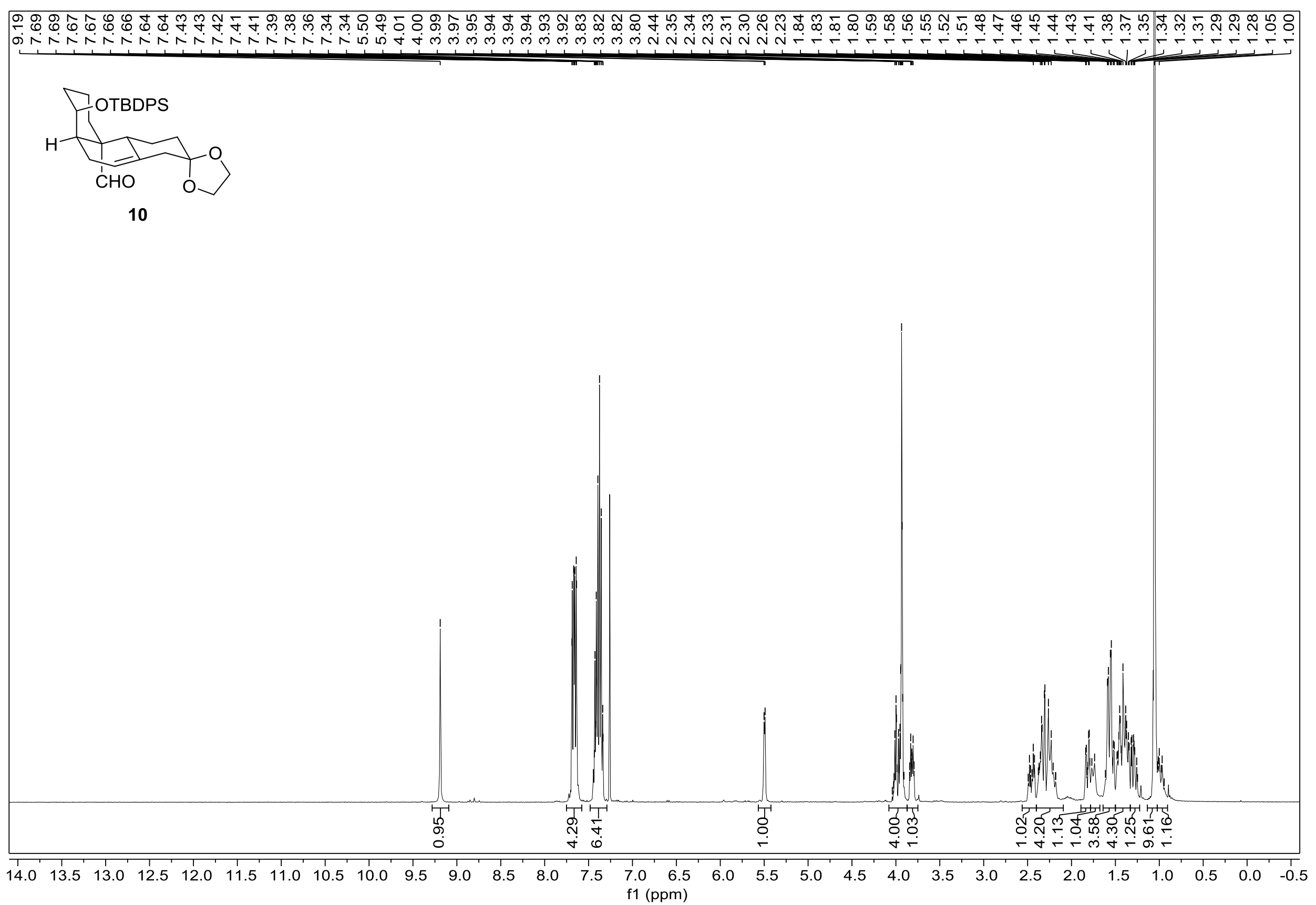


${ }^{13}$ C NMR Spectrum of 10 (101 MHz, acetone-d $\left.d_{6}\right)$

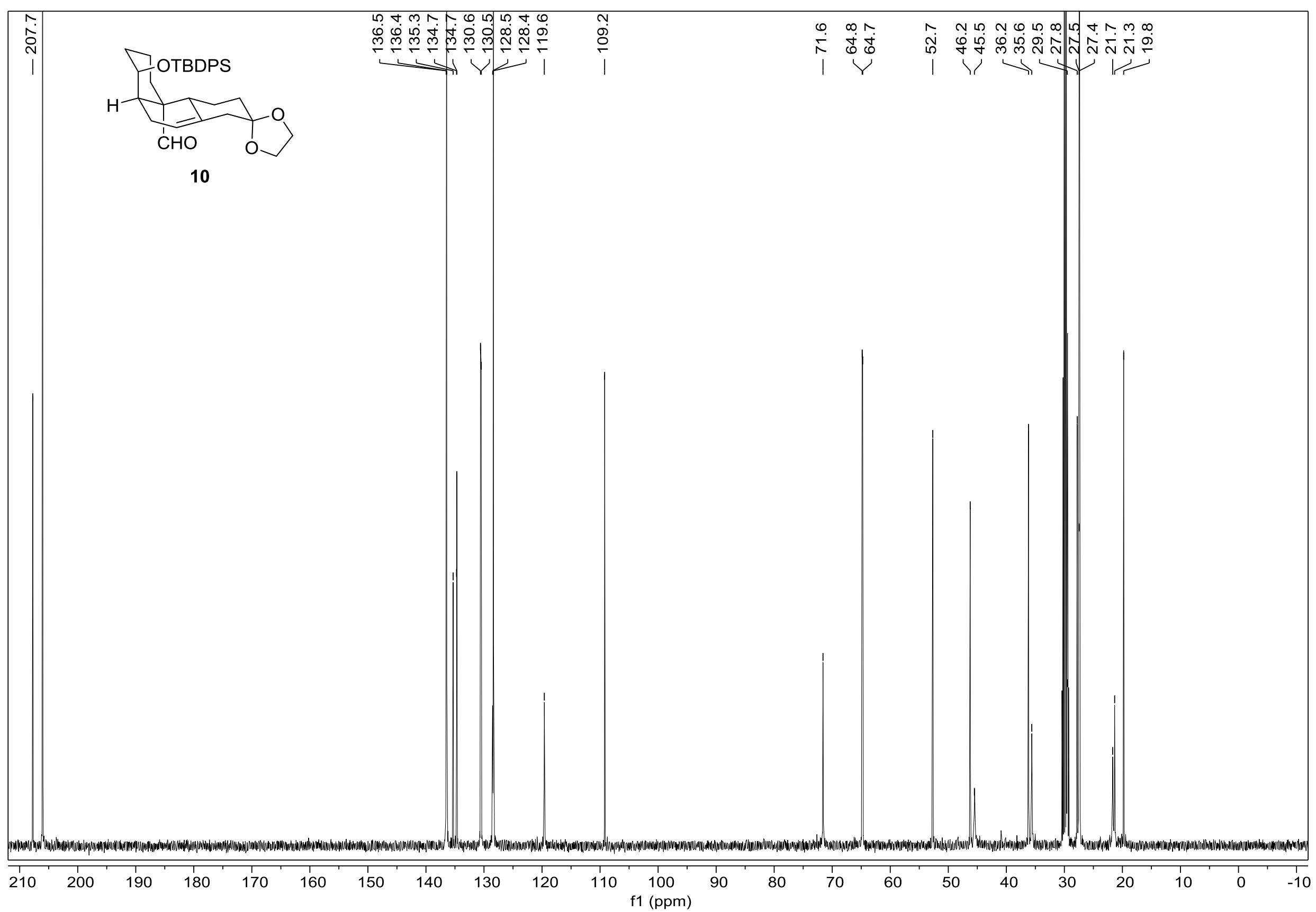


${ }^{1} \mathrm{H}$ NMR Spectrum of 15 (400 $\left.\mathrm{MHz}, \mathrm{CDCl}_{3}\right)$

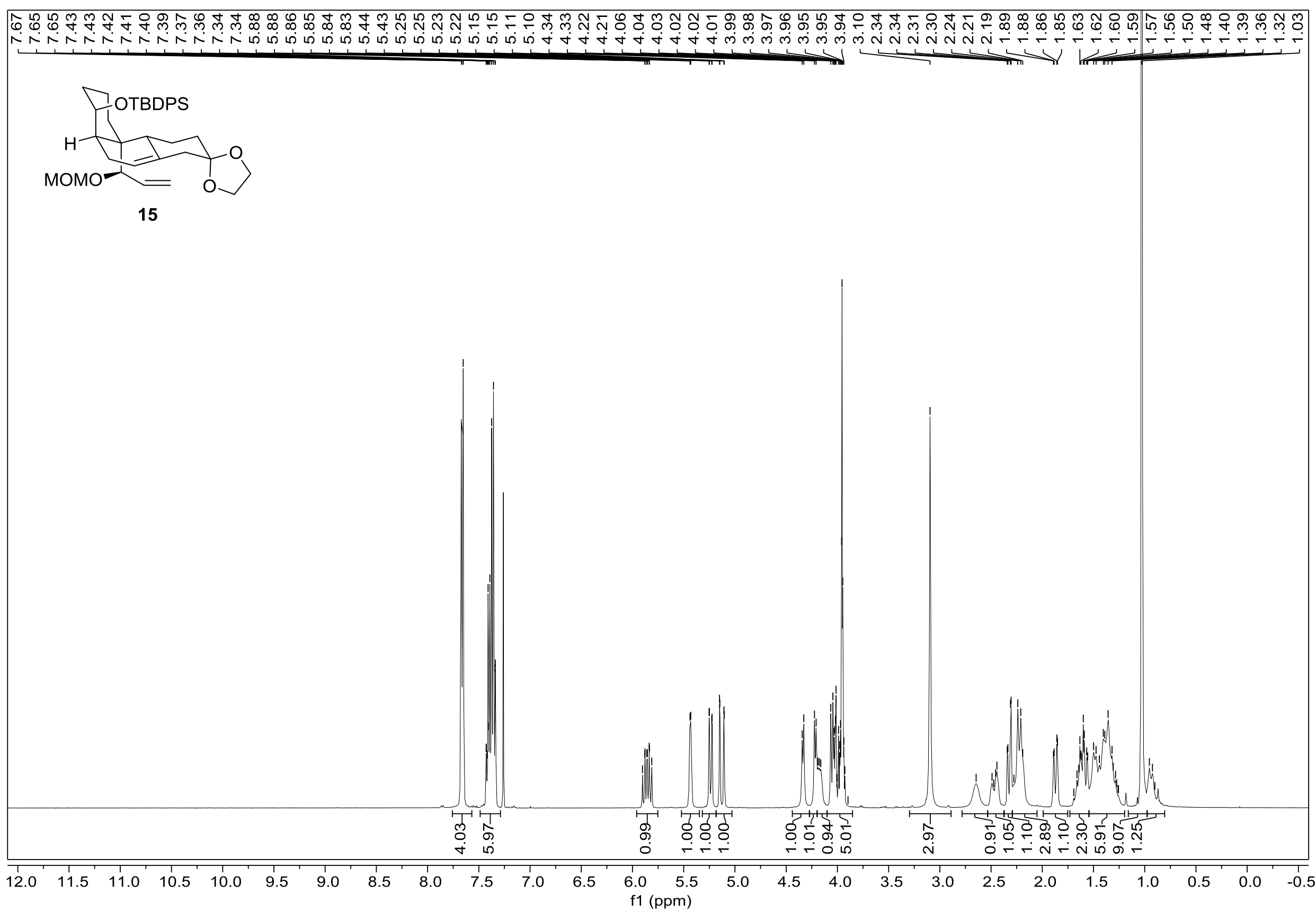


${ }^{13} \mathrm{C}$ NMR Spectrum of $15\left(101 \mathrm{MHz}, \mathrm{CDCl}_{3}\right)$

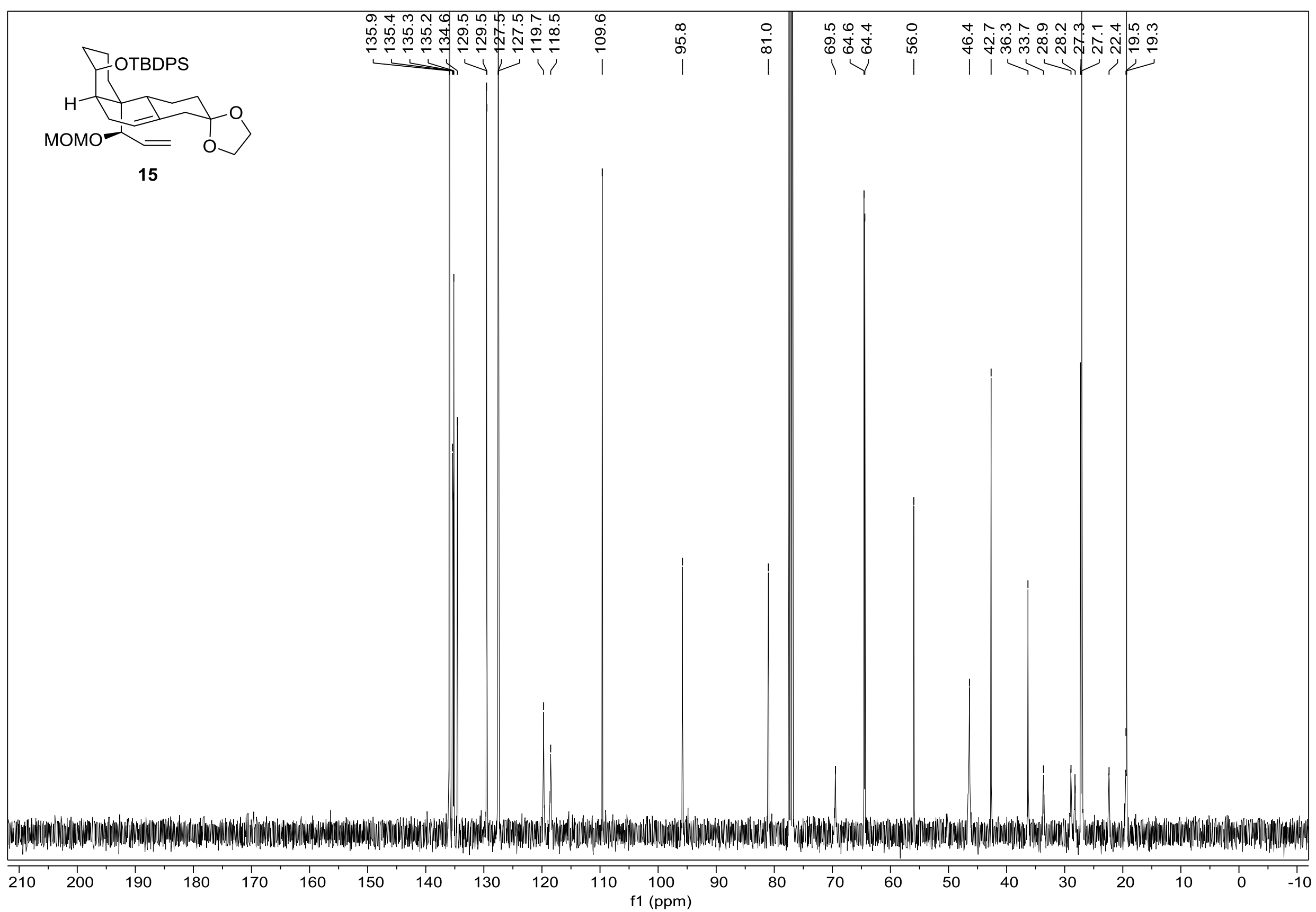


${ }^{1} \mathrm{H}$ NMR Spectrum of $9\left(400 \mathrm{MHz}, \mathrm{CDCl}_{3}\right)$

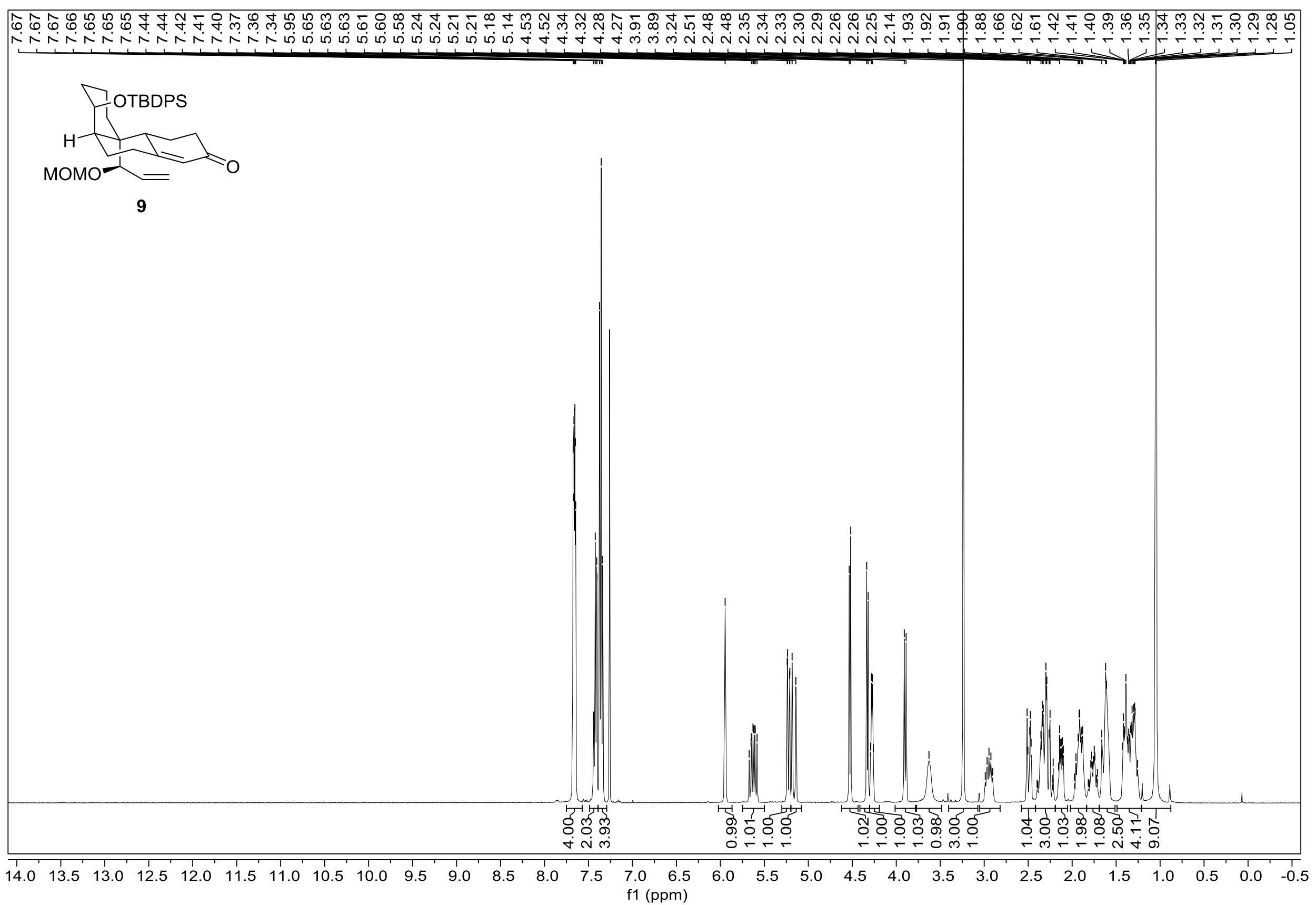


${ }^{13} \mathrm{C}$ NMR Spectrum of $9\left(101 \mathrm{MHz}, \mathrm{CDCl}_{3}\right)$

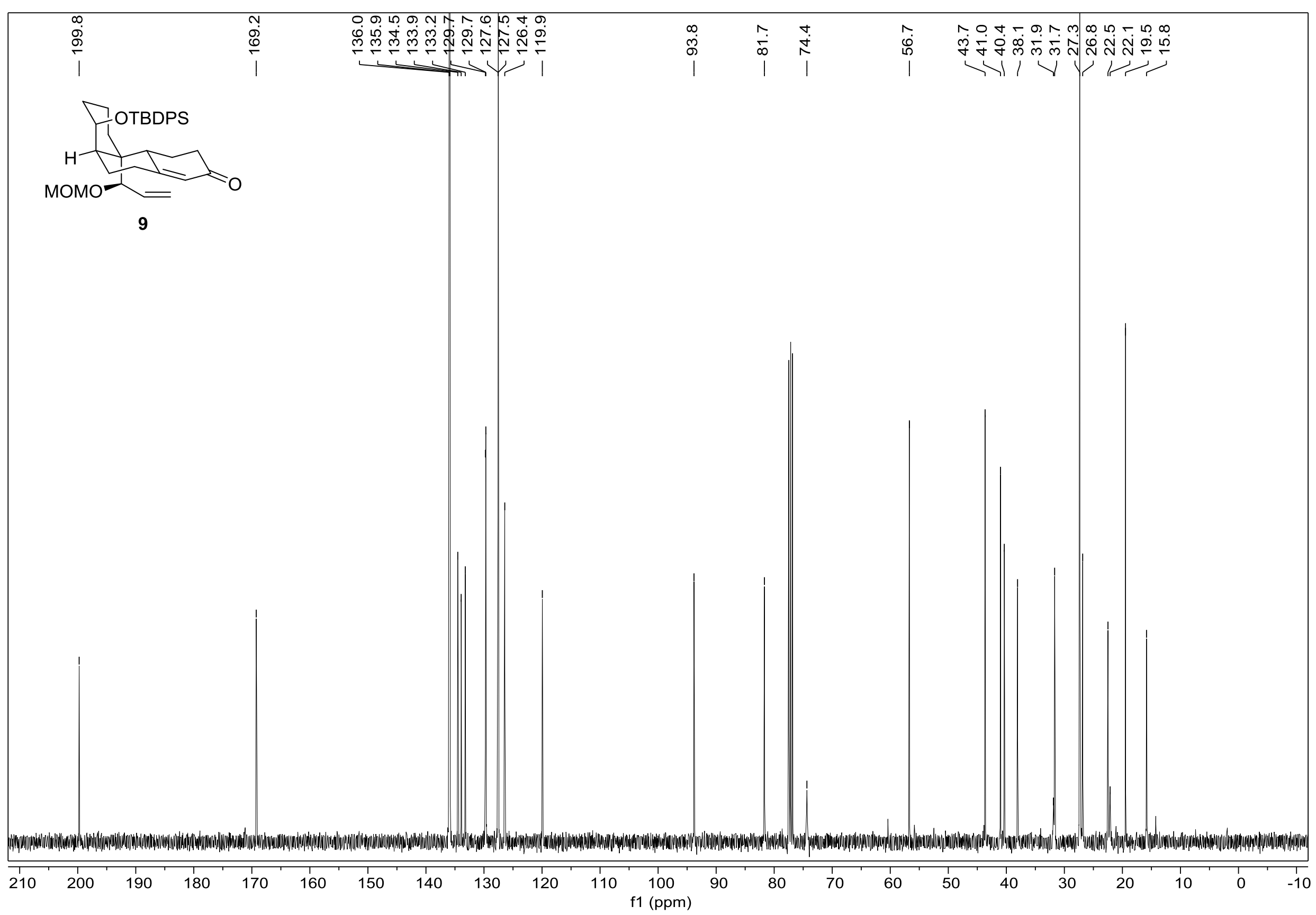


${ }^{1} \mathrm{H}$ NMR Spectrum of $16\left(500 \mathrm{MHz}, \mathrm{CDCl}_{3}\right)$

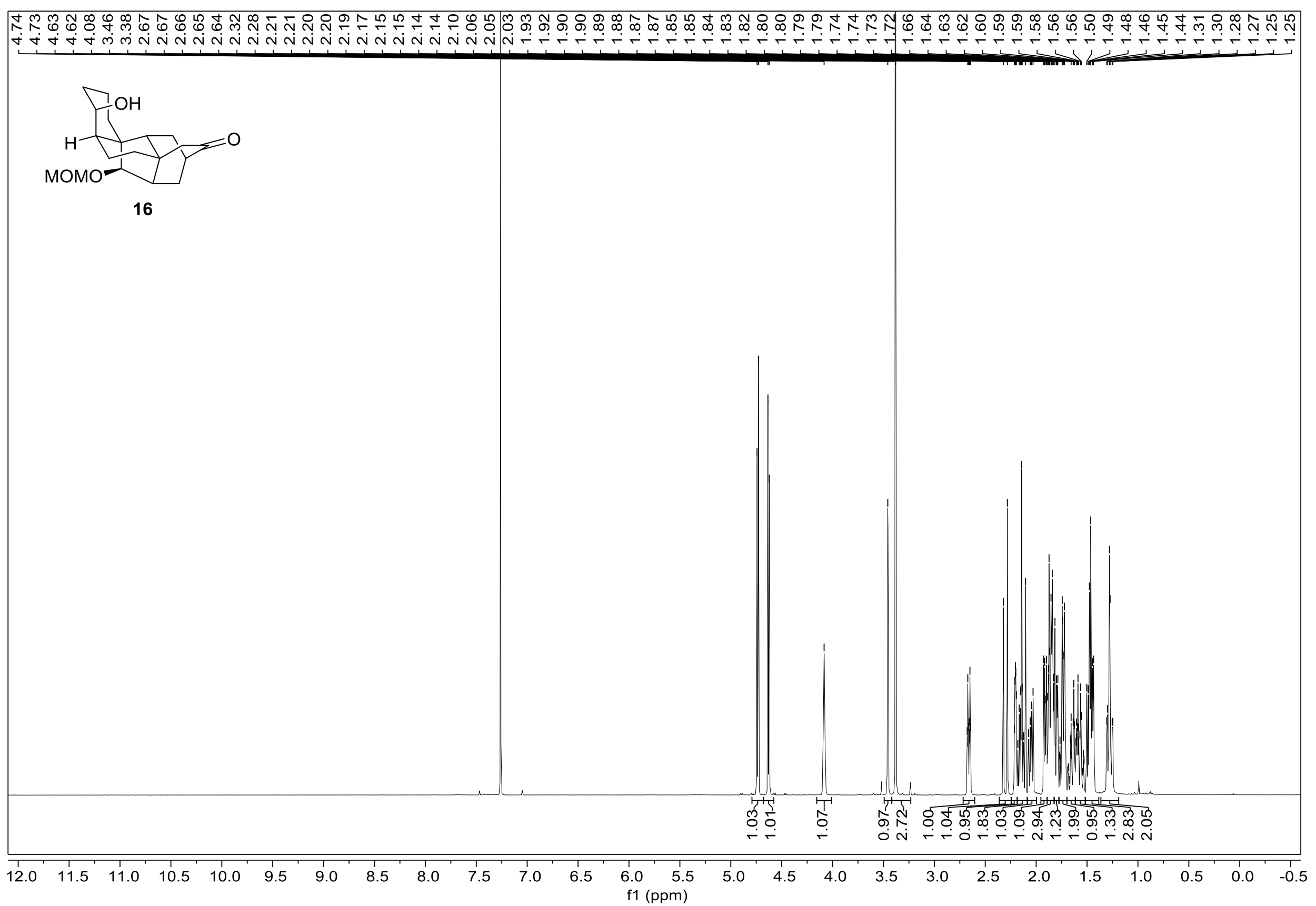


${ }^{13} \mathrm{C}$ NMR Spectrum of $16\left(126 \mathrm{MHz}, \mathrm{CDCl}_{3}\right)$

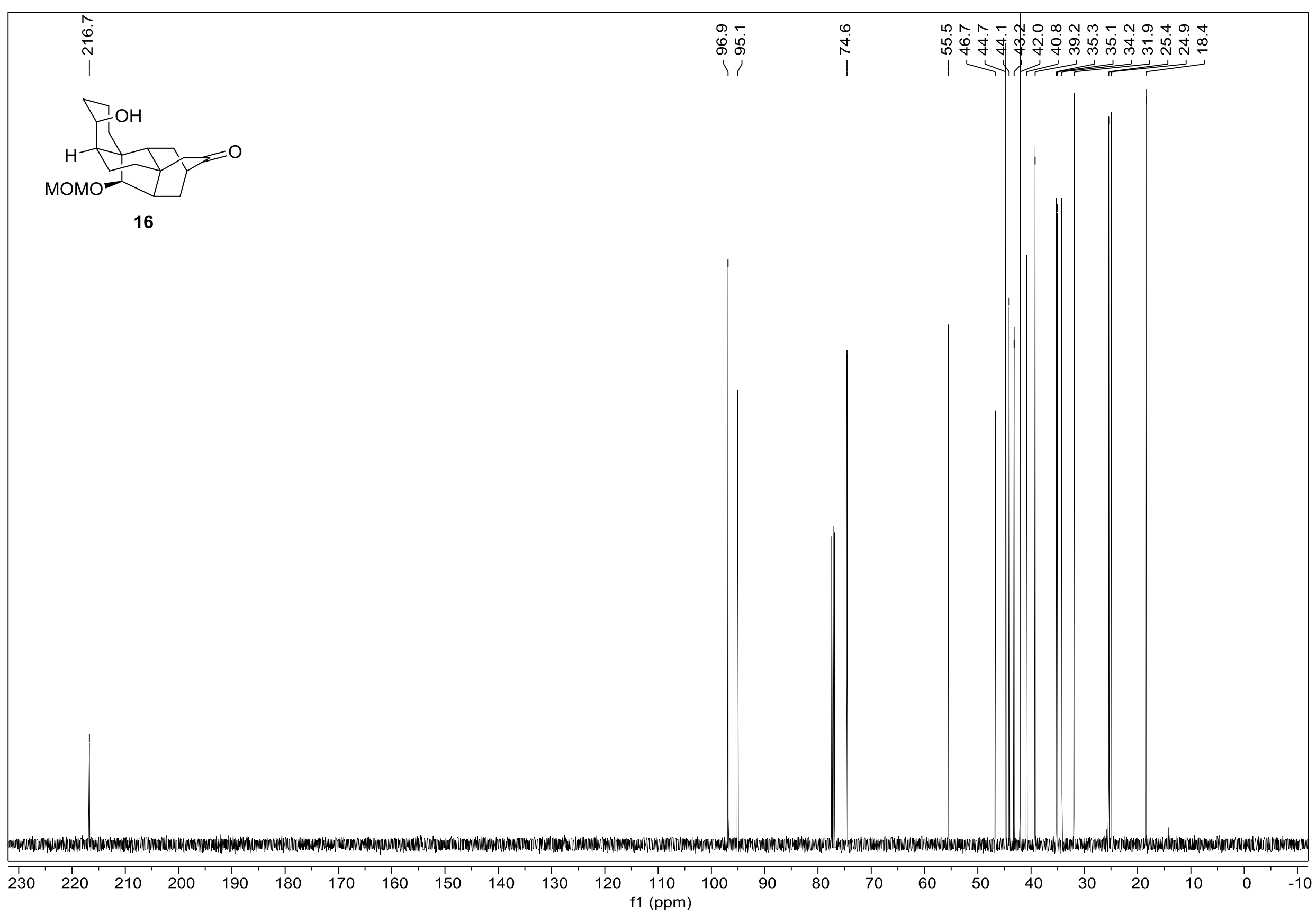


${ }^{1} \mathrm{H}$ NMR Spectrum of $8\left(500 \mathrm{MHz}, \mathrm{CDCl}_{3}\right)$

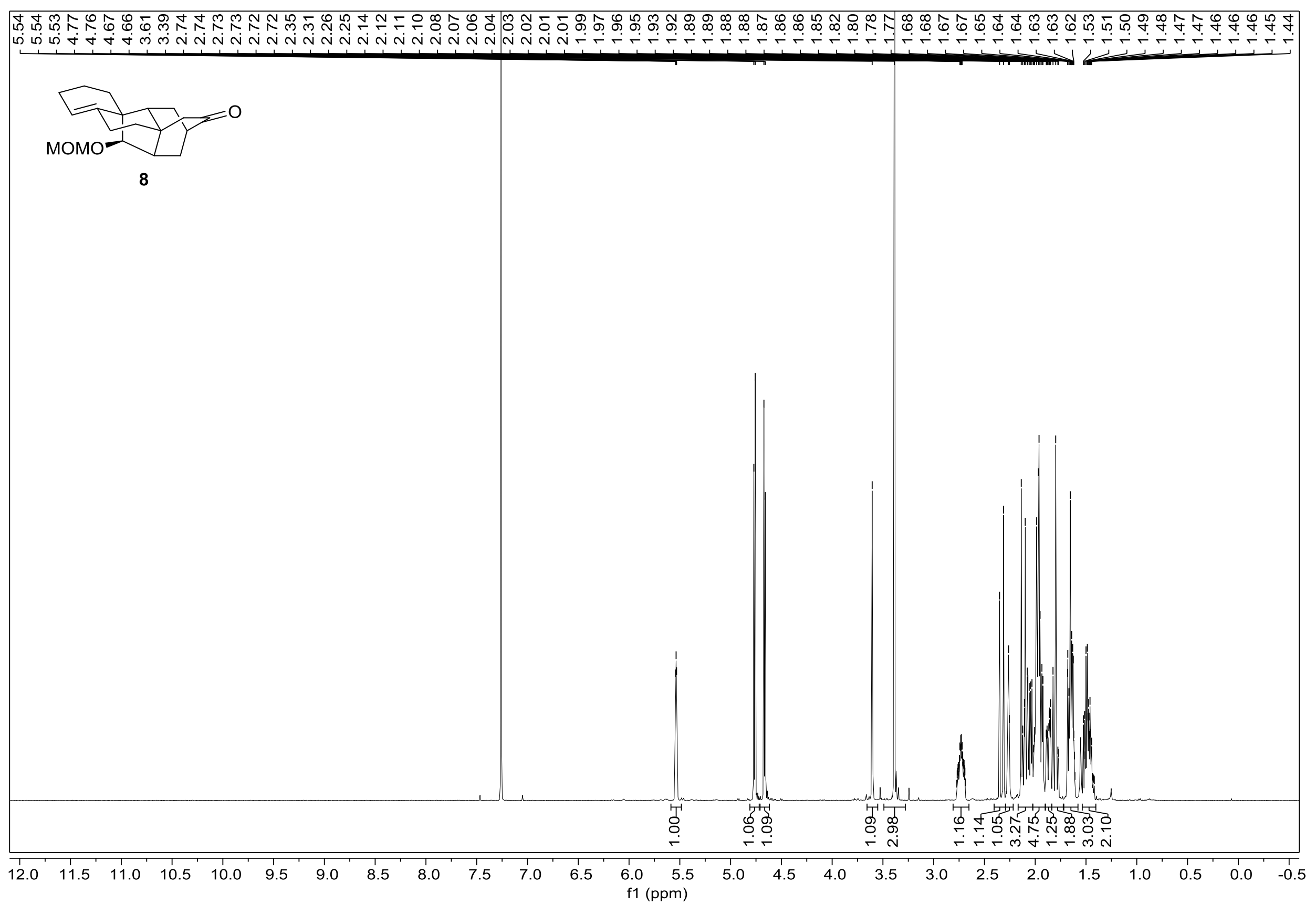


${ }^{13} \mathrm{C}$ NMR Spectrum of $8\left(126 \mathrm{MHz}, \mathrm{CDCl}_{3}\right)$

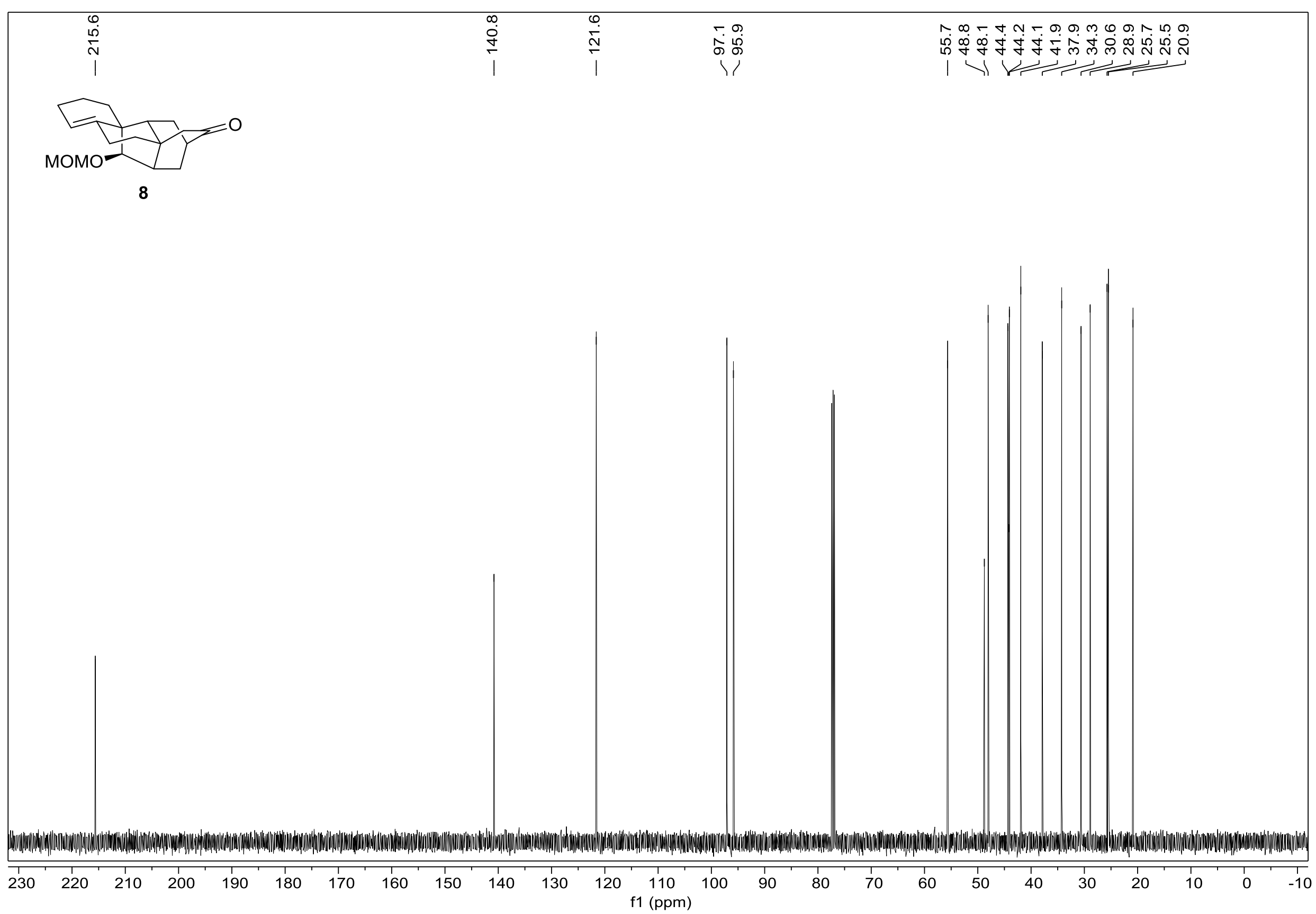


${ }^{1} \mathrm{H}$ NMR Spectrum of 7 (400 $\left.\mathrm{MHz}, \mathrm{CDCl}_{3}\right)$

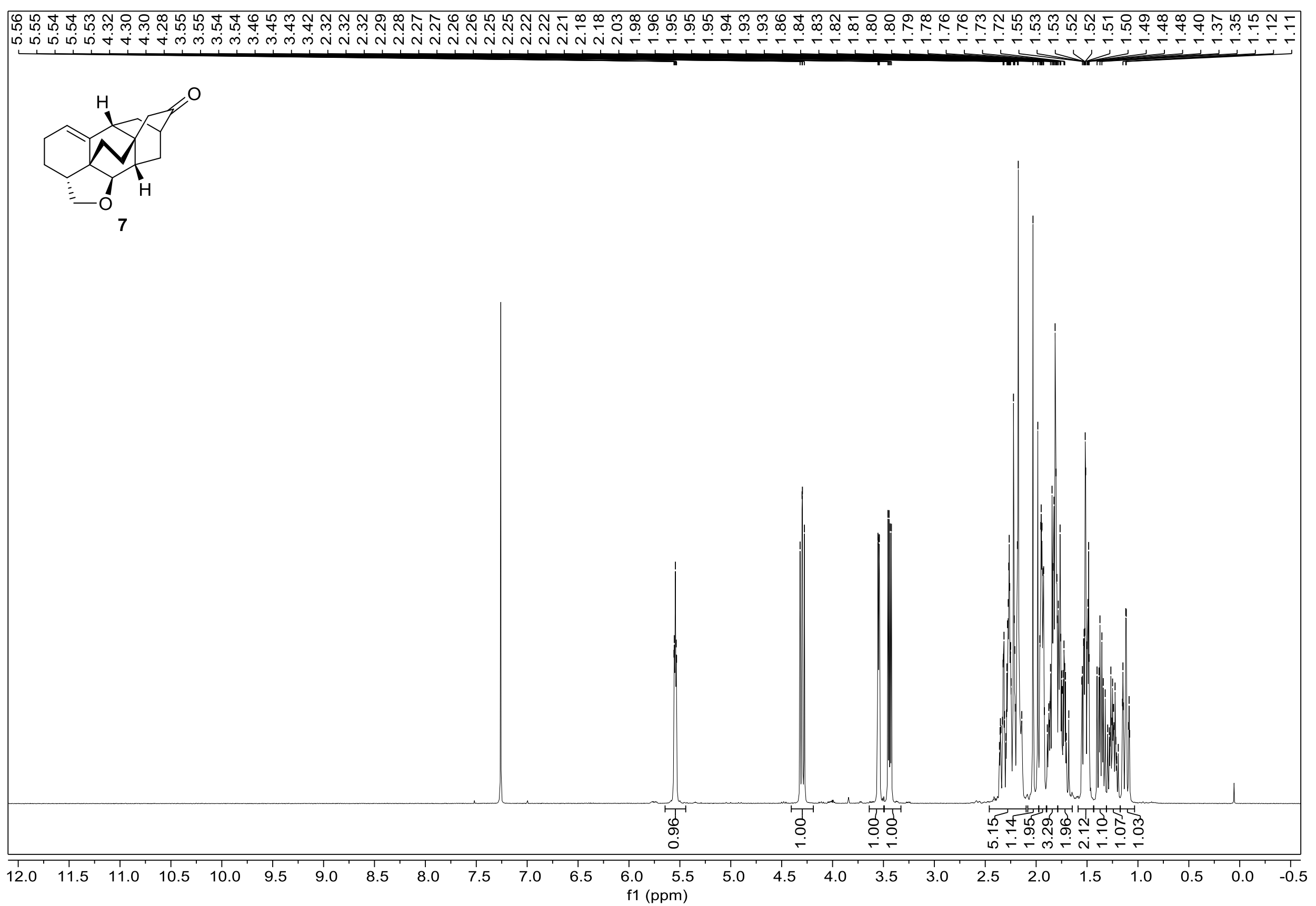


${ }^{13} \mathrm{C}$ NMR Spectrum of 7 (126 $\left.\mathrm{MHz}, \mathrm{CDCl}_{3}\right)$

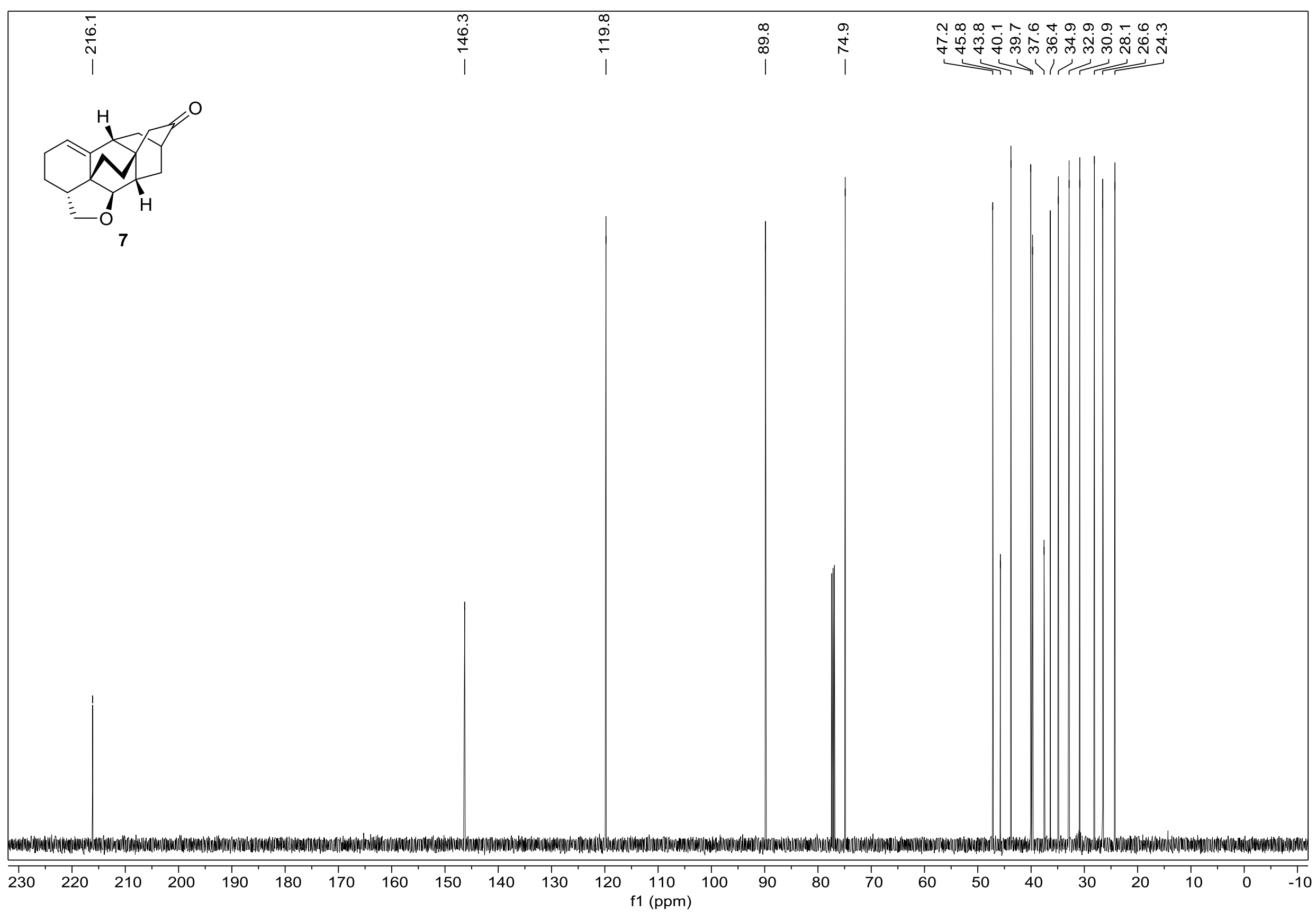


${ }^{1} \mathrm{H}$ NMR Spectrum of 19 (400 $\left.\mathrm{MHz}, \mathrm{CDCl}_{3}\right)$

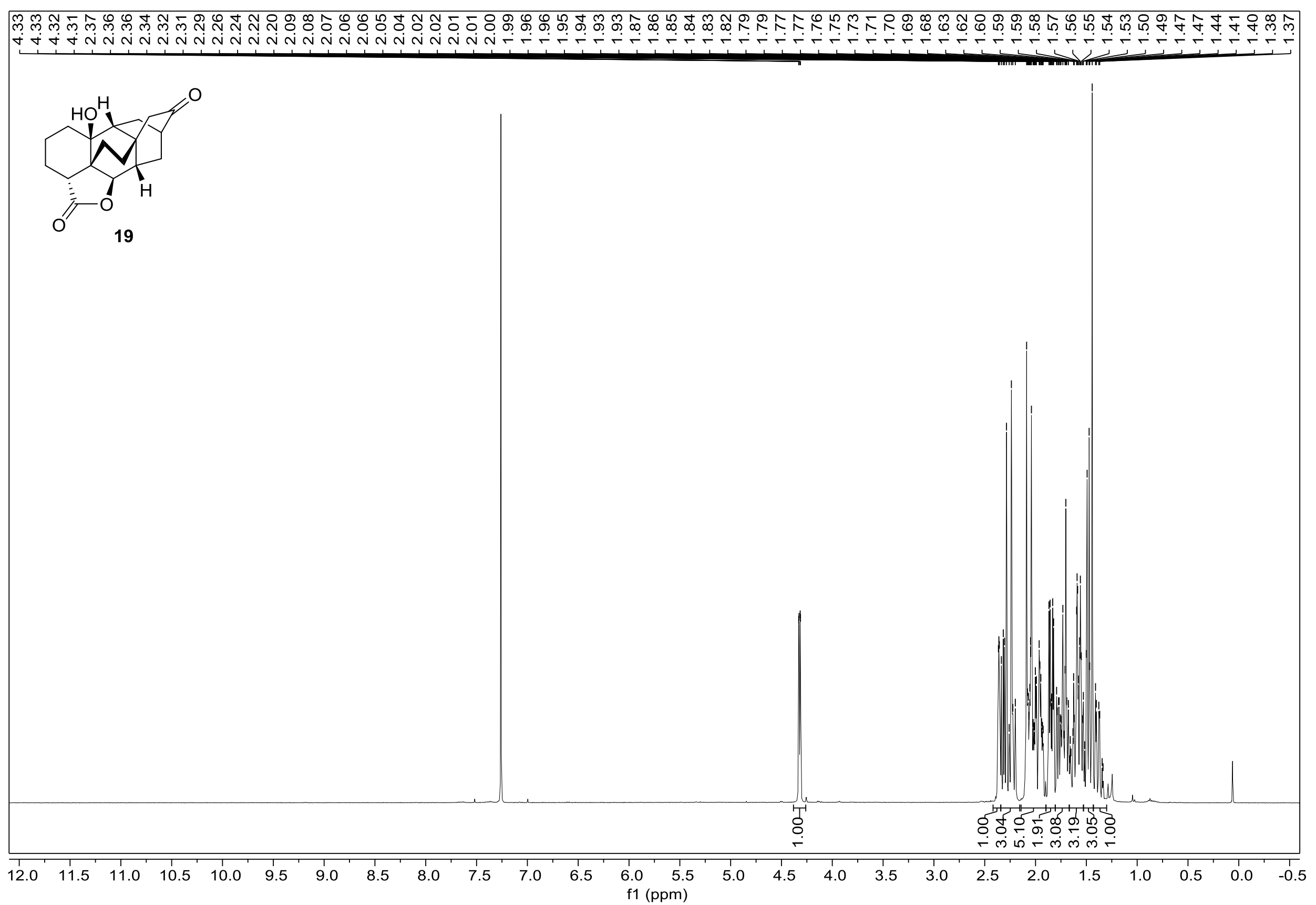


${ }^{13} \mathrm{C}$ NMR Spectrum of 19 (126 $\left.\mathrm{MHz}, \mathrm{CDCl}_{3}\right)$

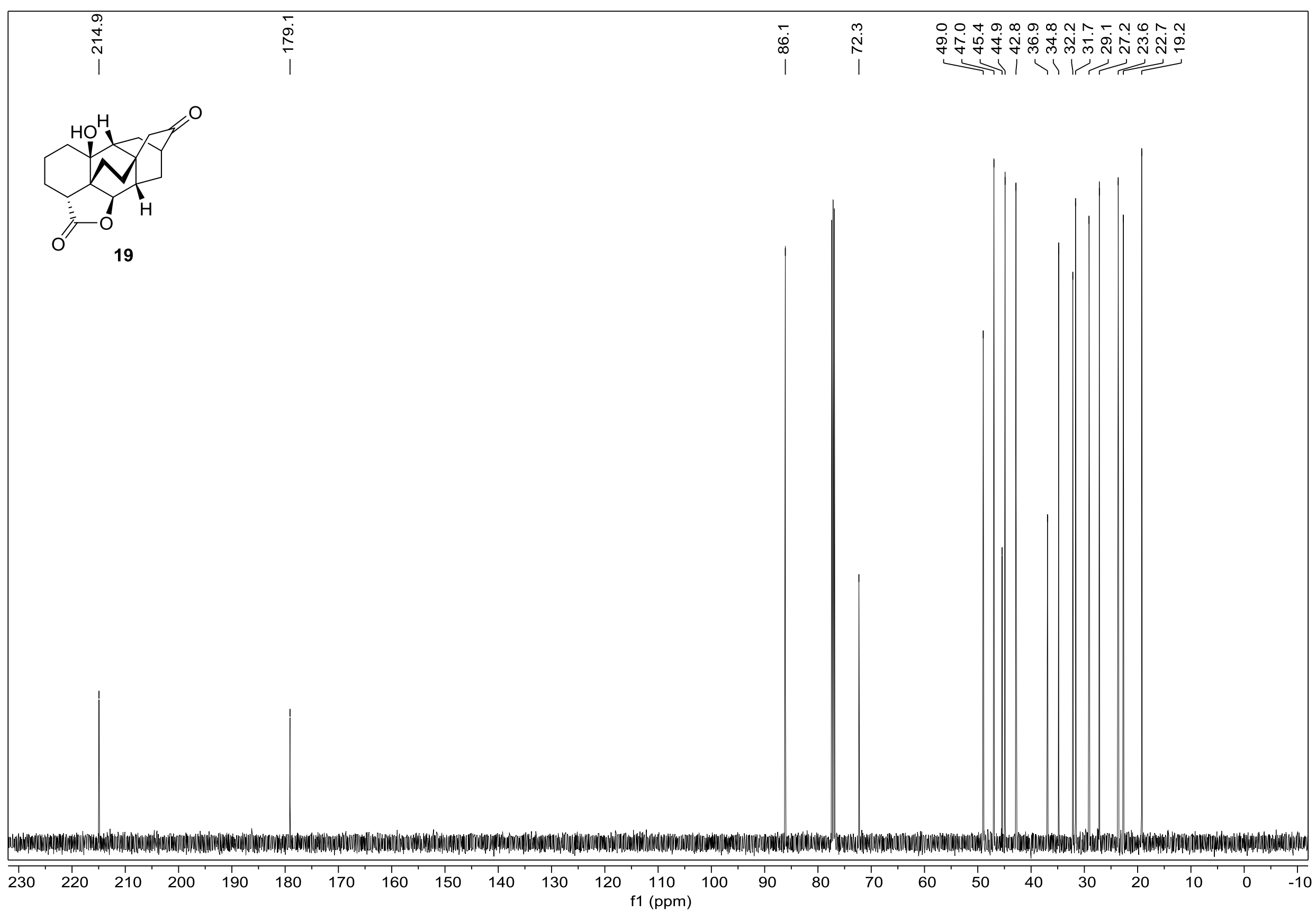




\section{${ }^{1} \mathrm{H}$ NMR Spectrum of $20\left(500 \mathrm{MHz}, \mathrm{CDCl}_{3}\right)$}

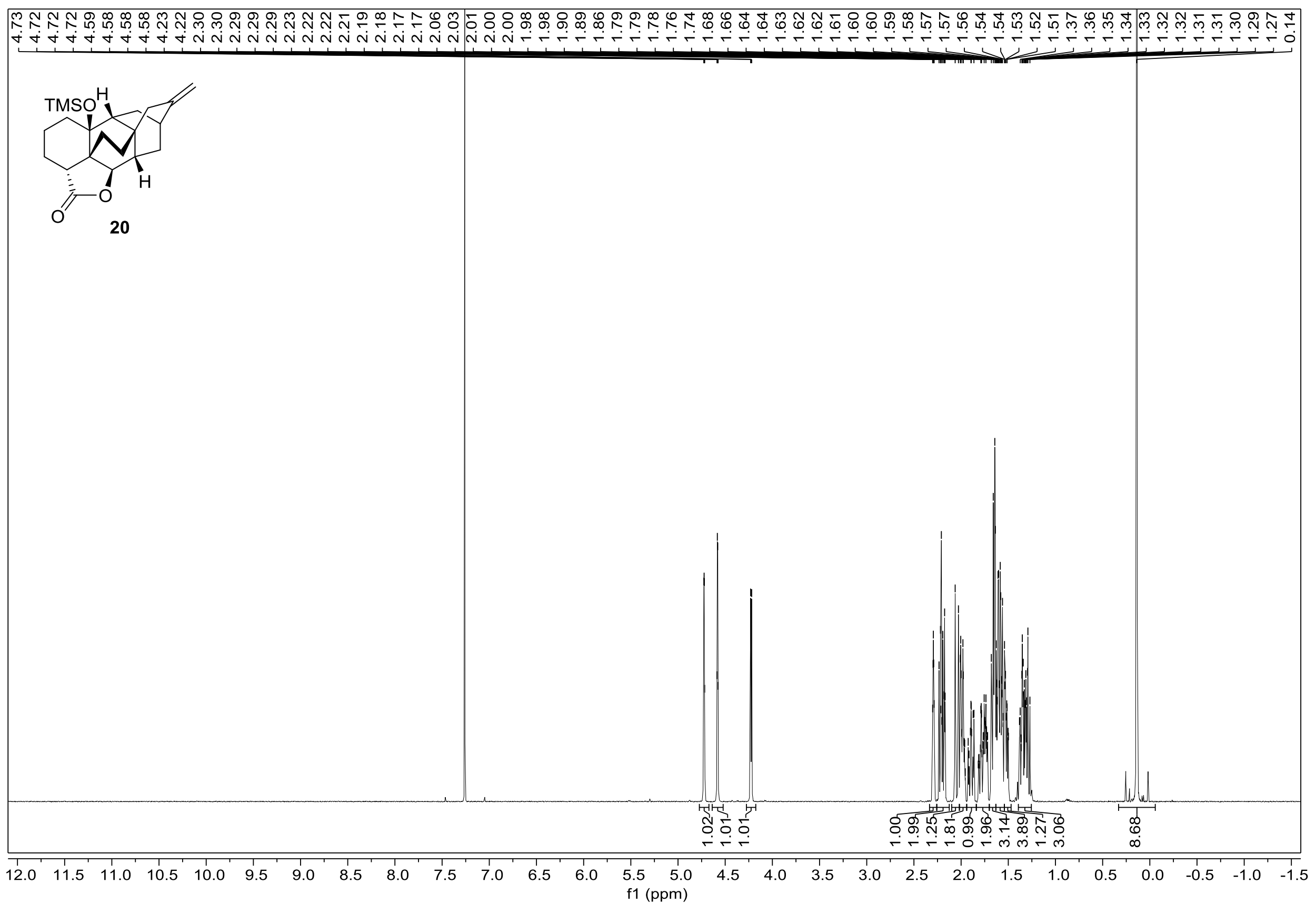


${ }^{13} \mathrm{C}$ NMR Spectrum of $20\left(126 \mathrm{MHz}, \mathrm{CDCl}_{3}\right)$

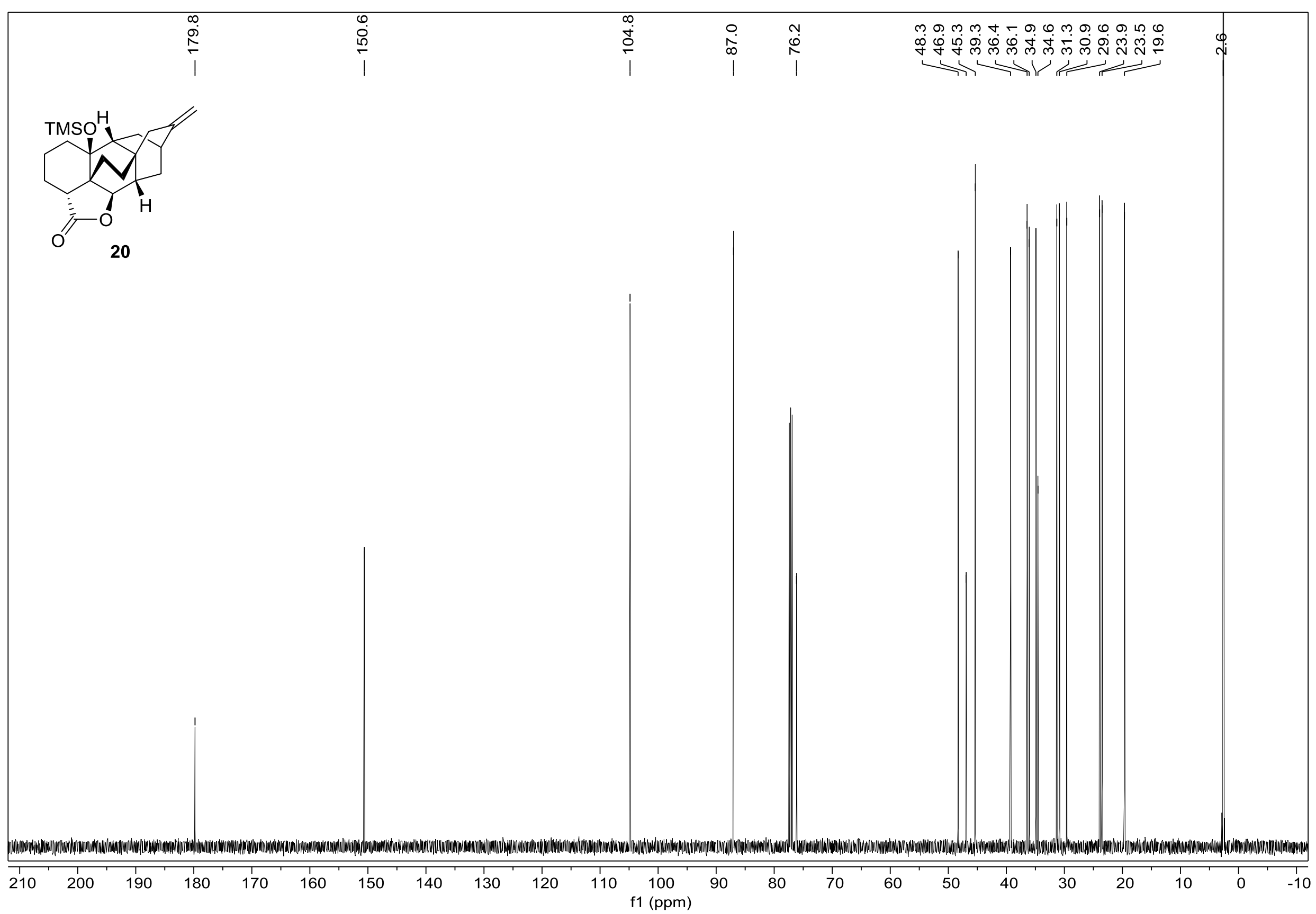


${ }^{1} \mathrm{H}$ NMR Spectrum of $21\left(500 \mathrm{MHz}, \mathrm{CDCl}_{3}\right)$

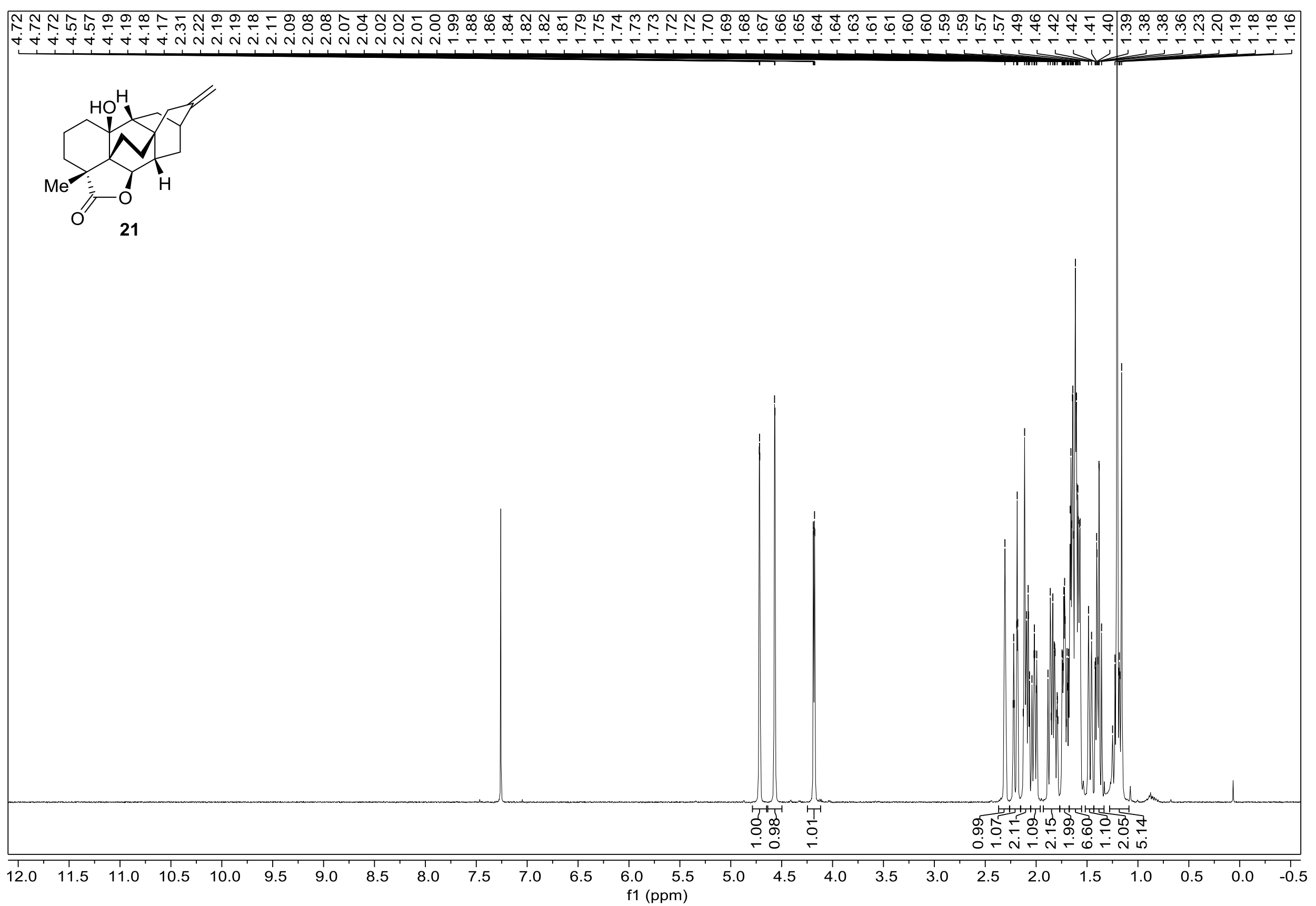


${ }^{13} \mathrm{C}$ NMR Spectrum of $21\left(126 \mathrm{MHz} \mathrm{CDCl}_{3}\right)$

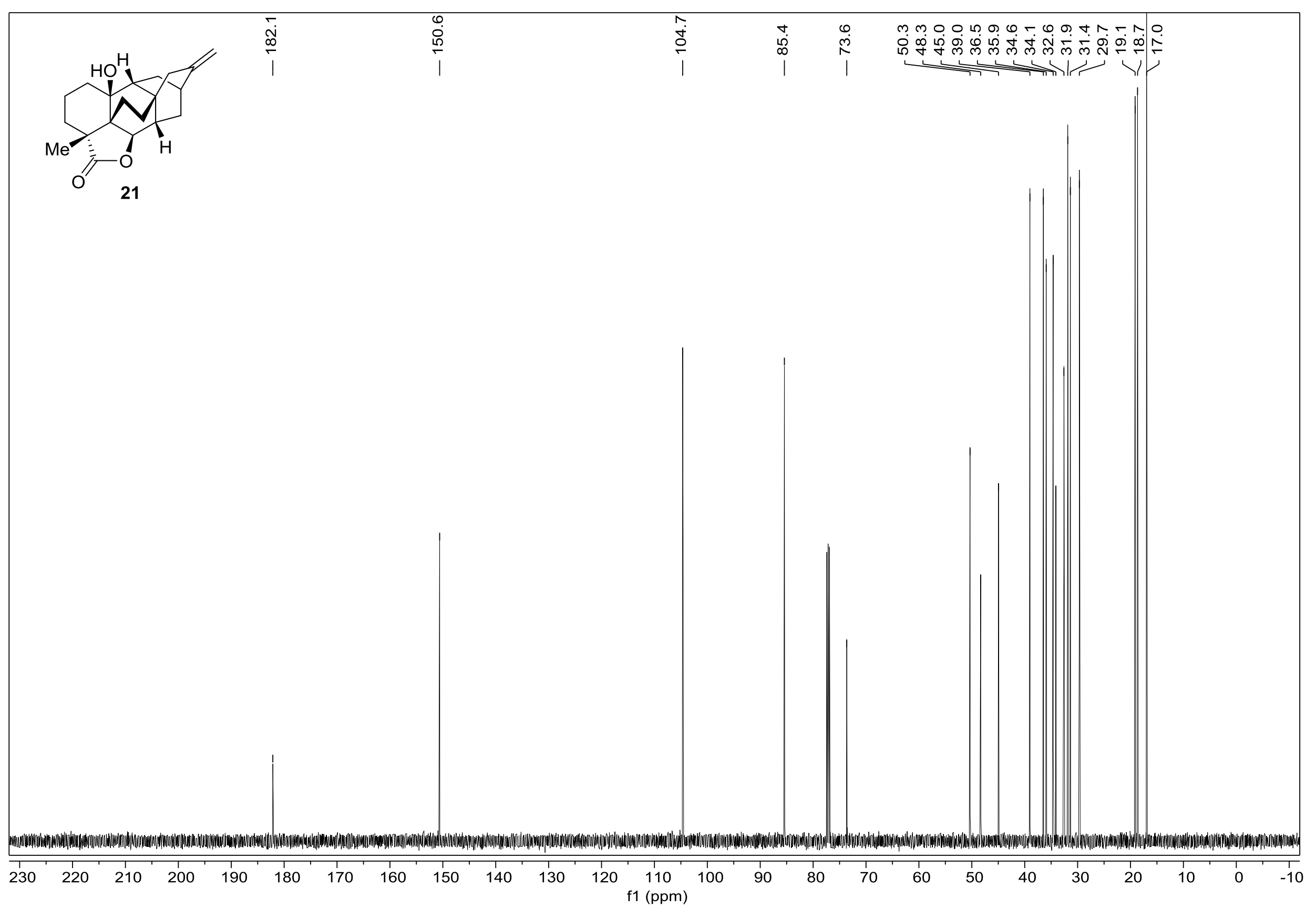




\section{${ }^{1} \mathrm{H}$ NMR Spectrum of $22\left(400 \mathrm{MHz}, \mathrm{CDCl}_{3}\right)$}

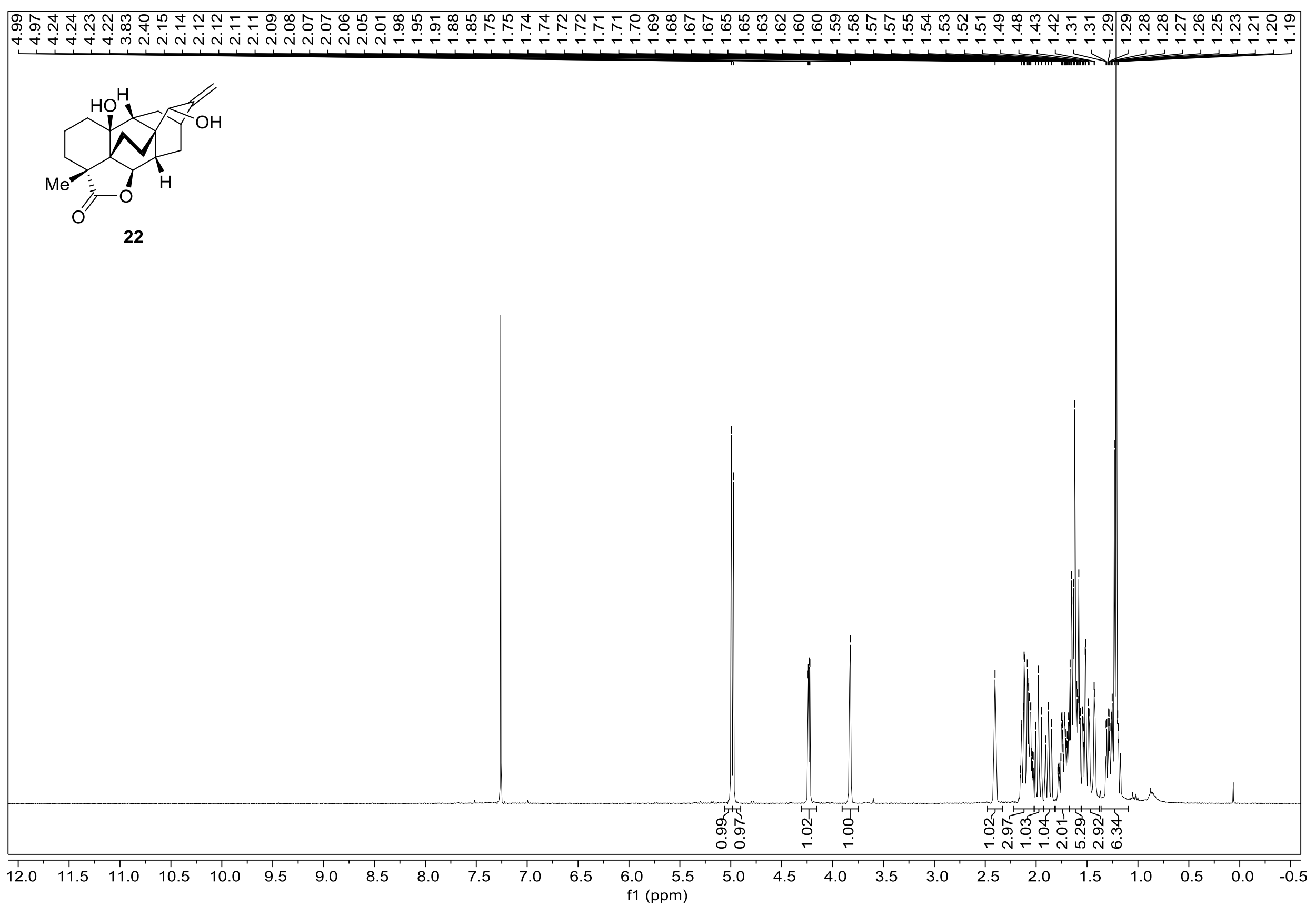


${ }^{13}$ C NMR Spectrum of 22 (126 MHz, acetone-d $\left.d_{6}\right)$

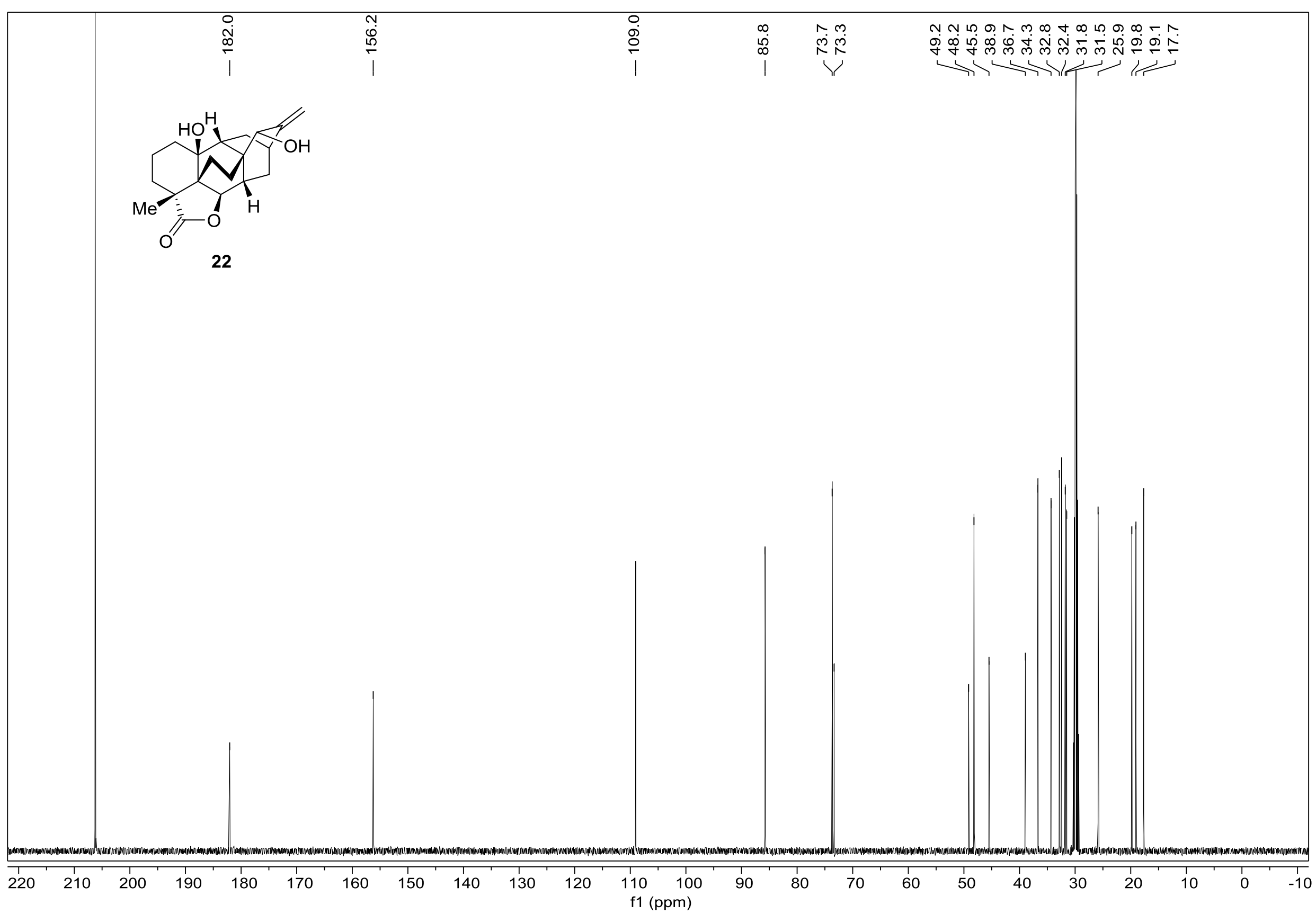


${ }^{1} \mathrm{H}$ NMR Spectrum of $6\left(400 \mathrm{MHz}, \mathrm{CDCl}_{3}\right)$

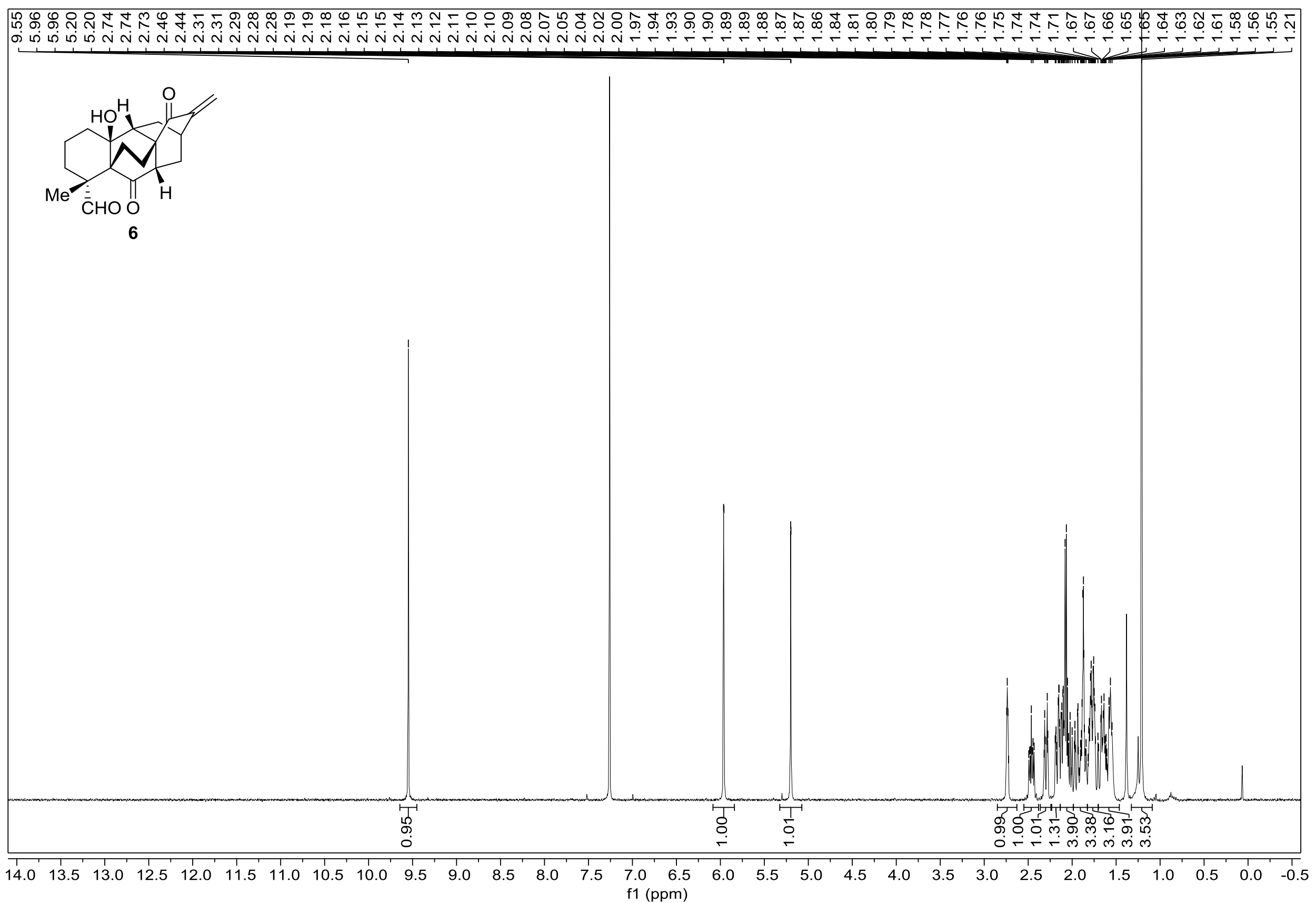


${ }^{13} \mathrm{C}$ NMR Spectrum of $6\left(126 \mathrm{MHz}, \mathrm{CDCl}_{3}\right)$

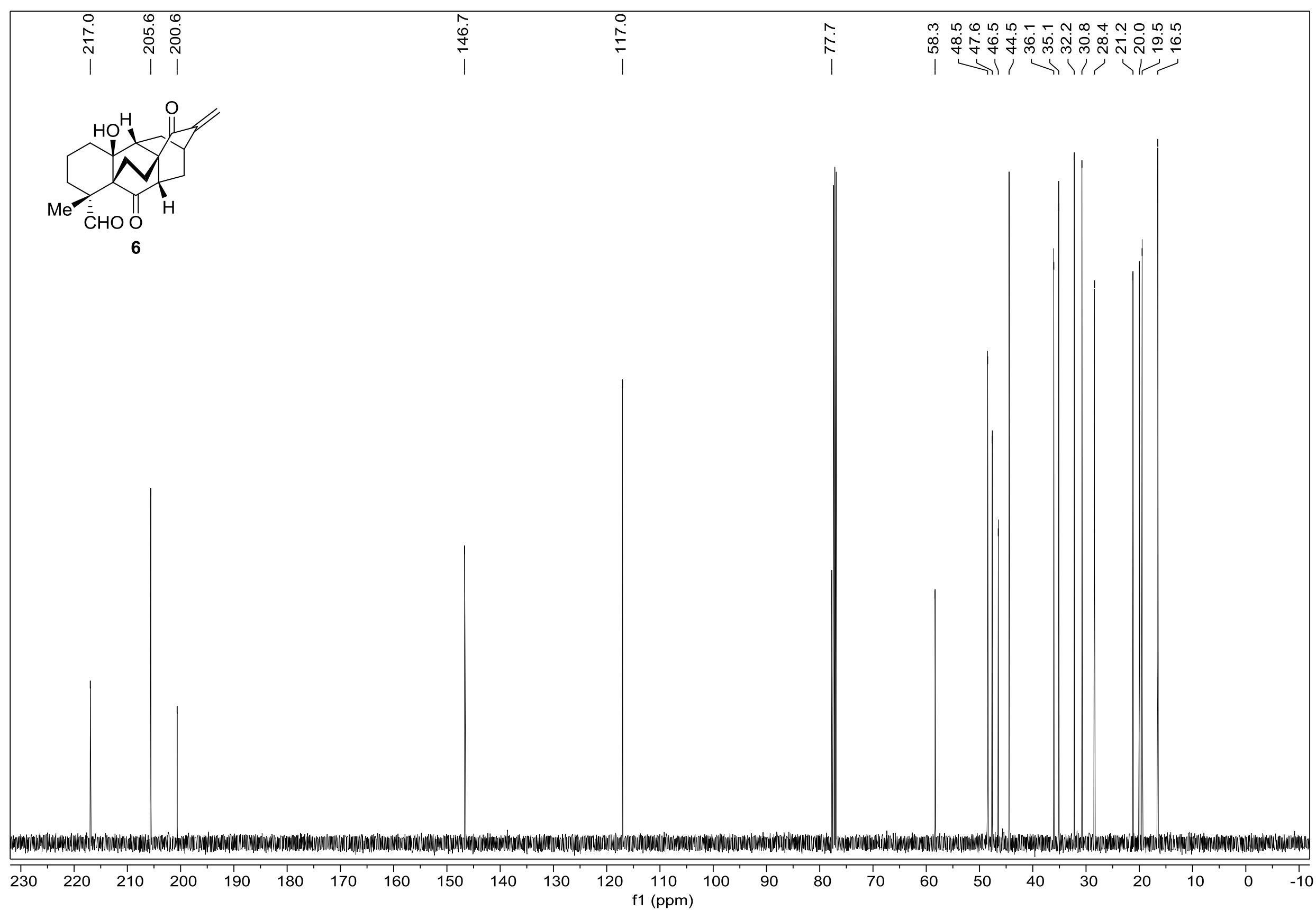


${ }^{1} \mathrm{H}$ NMR Spectrum of 23 (400 MHz, methanol-d 4 )

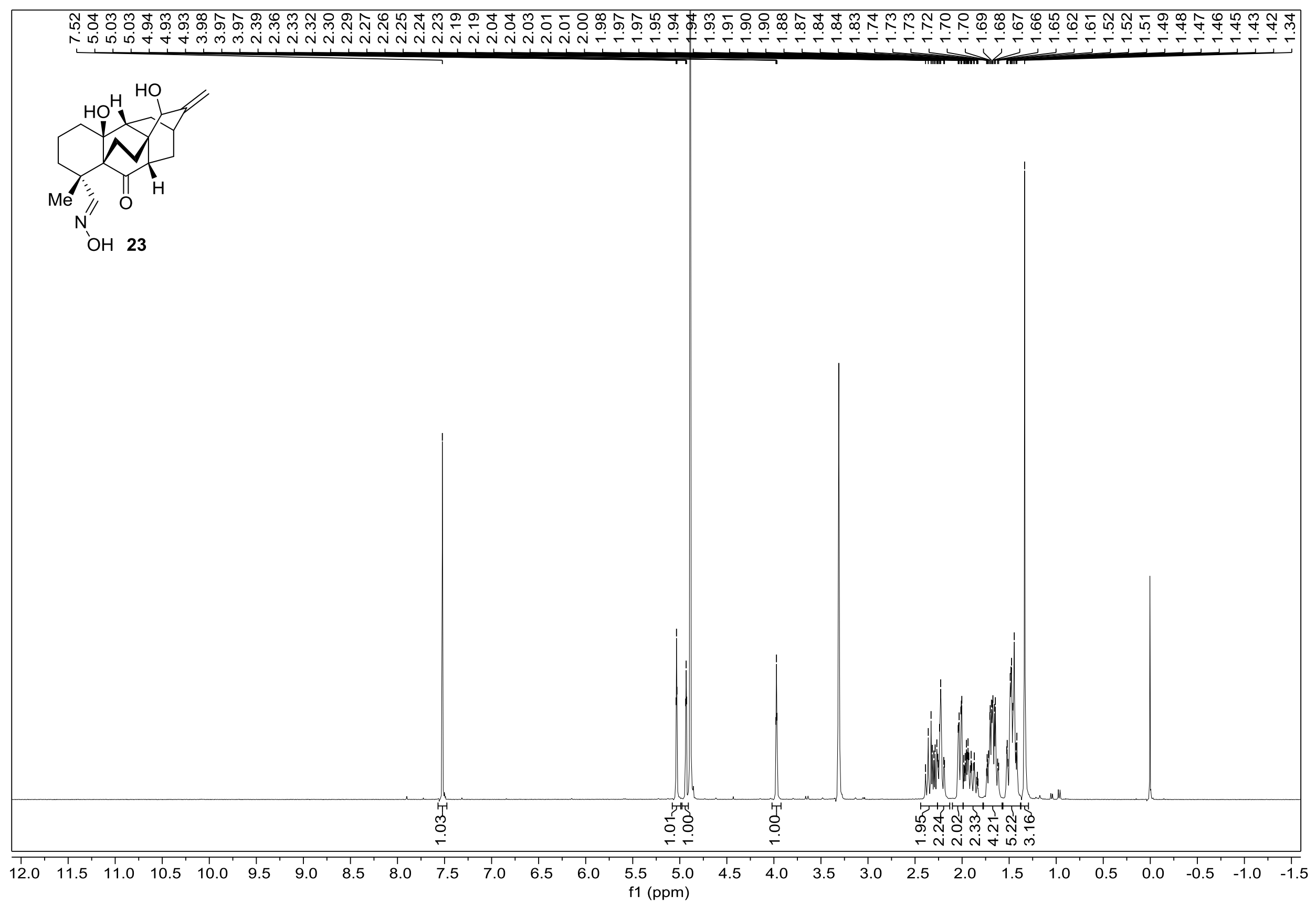


${ }^{13}$ C NMR Spectrum of 23 (126 MHz, methanol-d 4 )

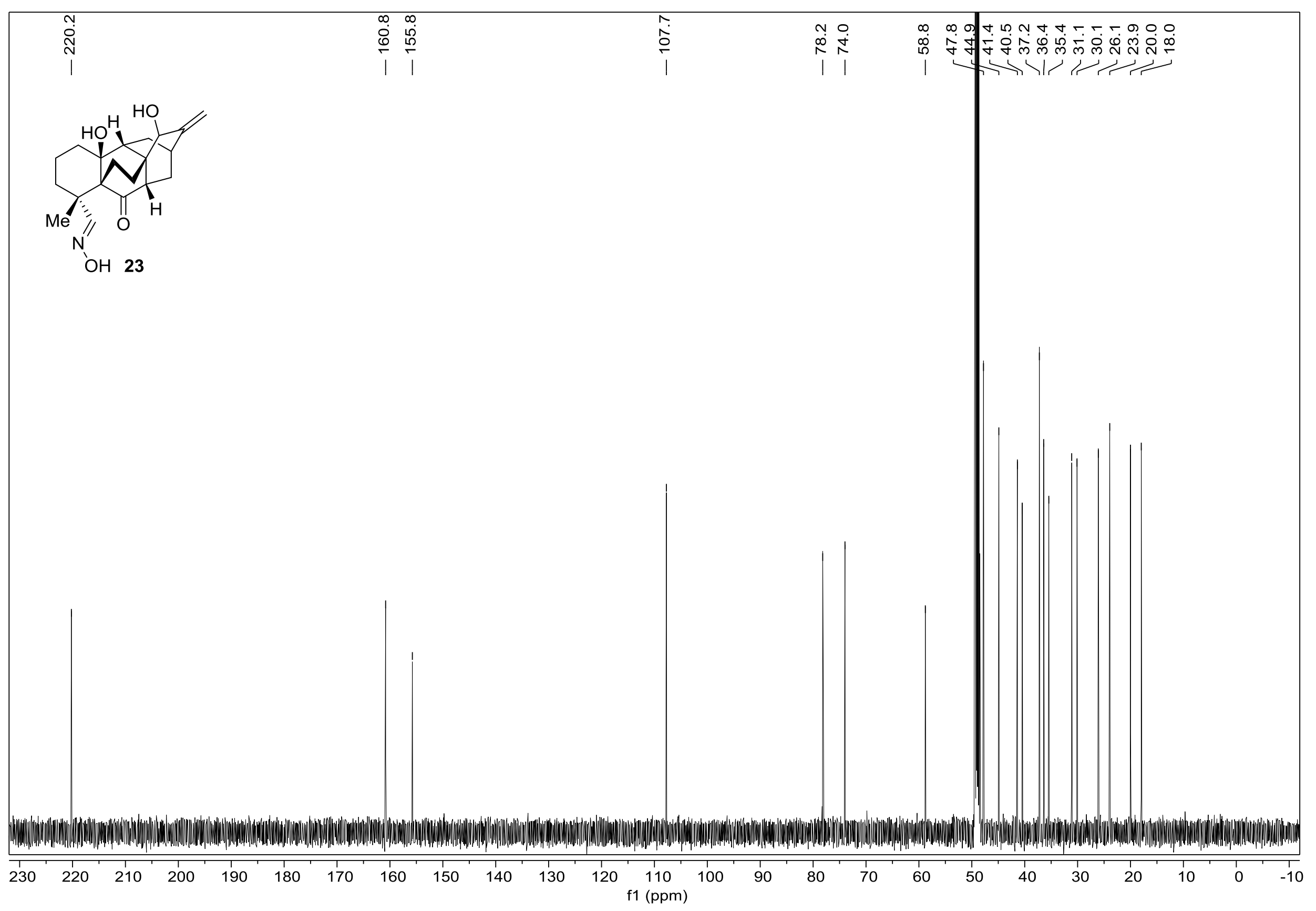


${ }^{1} \mathrm{H}$ NMR Spectrum of 3 (500 $\left.\mathrm{MHz}, \mathrm{CDCl}_{3}\right)$

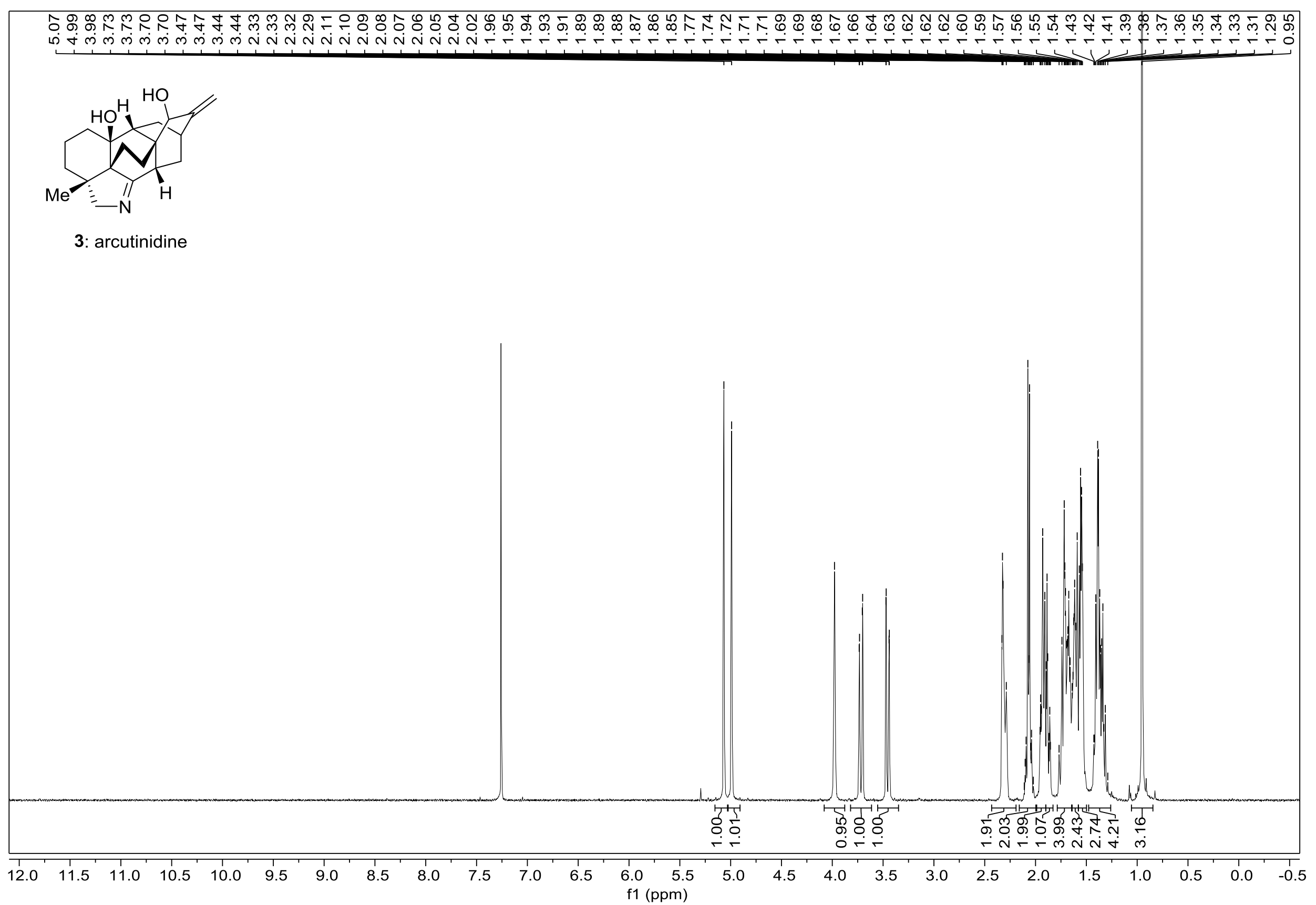


${ }^{13} \mathrm{C}$ NMR Spectrum of $3\left(126 \mathrm{MHz}, \mathrm{CDCl}_{3}\right)$

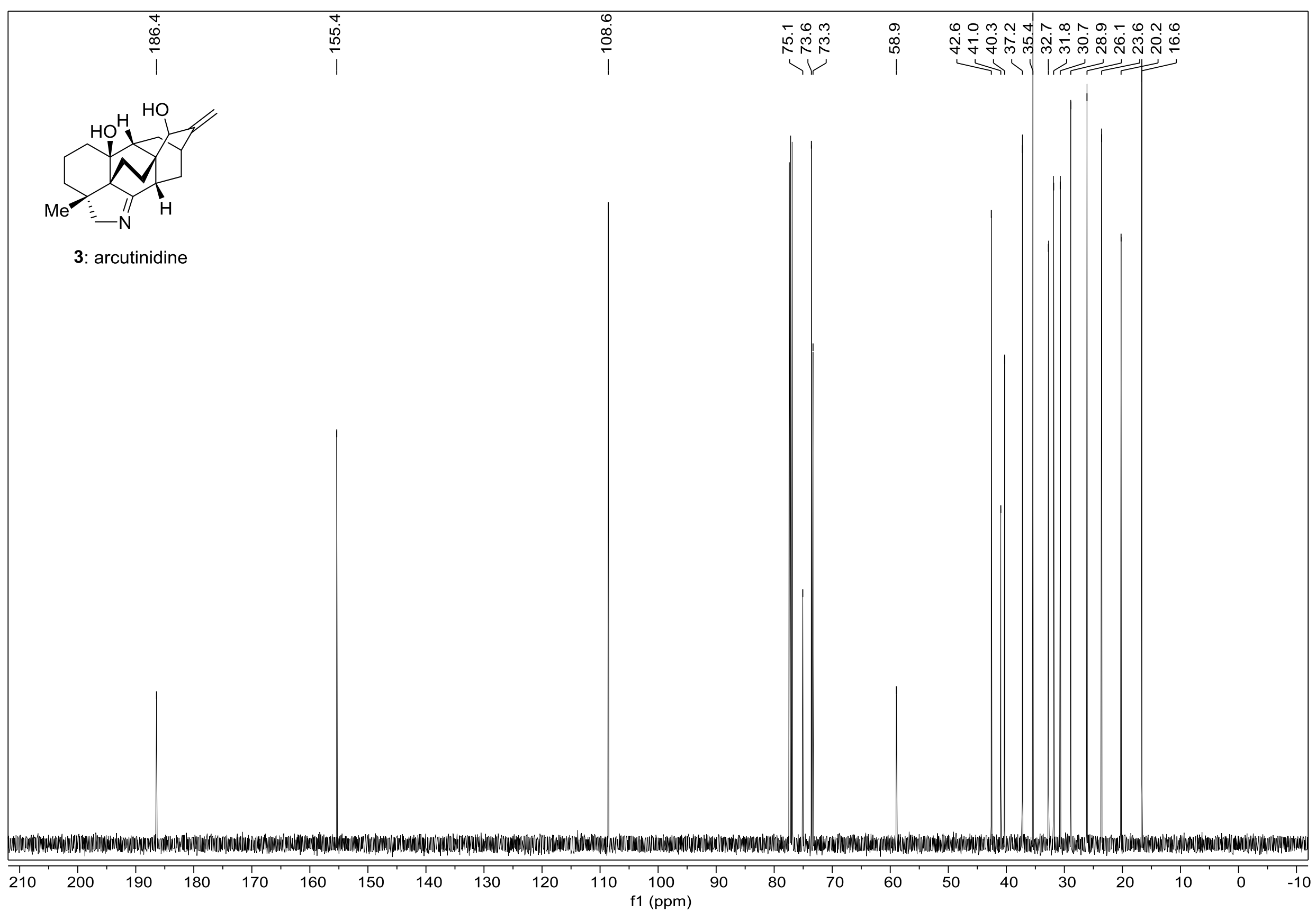


${ }^{1} \mathrm{H}$ NMR Spectrum of $2\left(400 \mathrm{MHz}, \mathrm{CDCl}_{3}\right)$

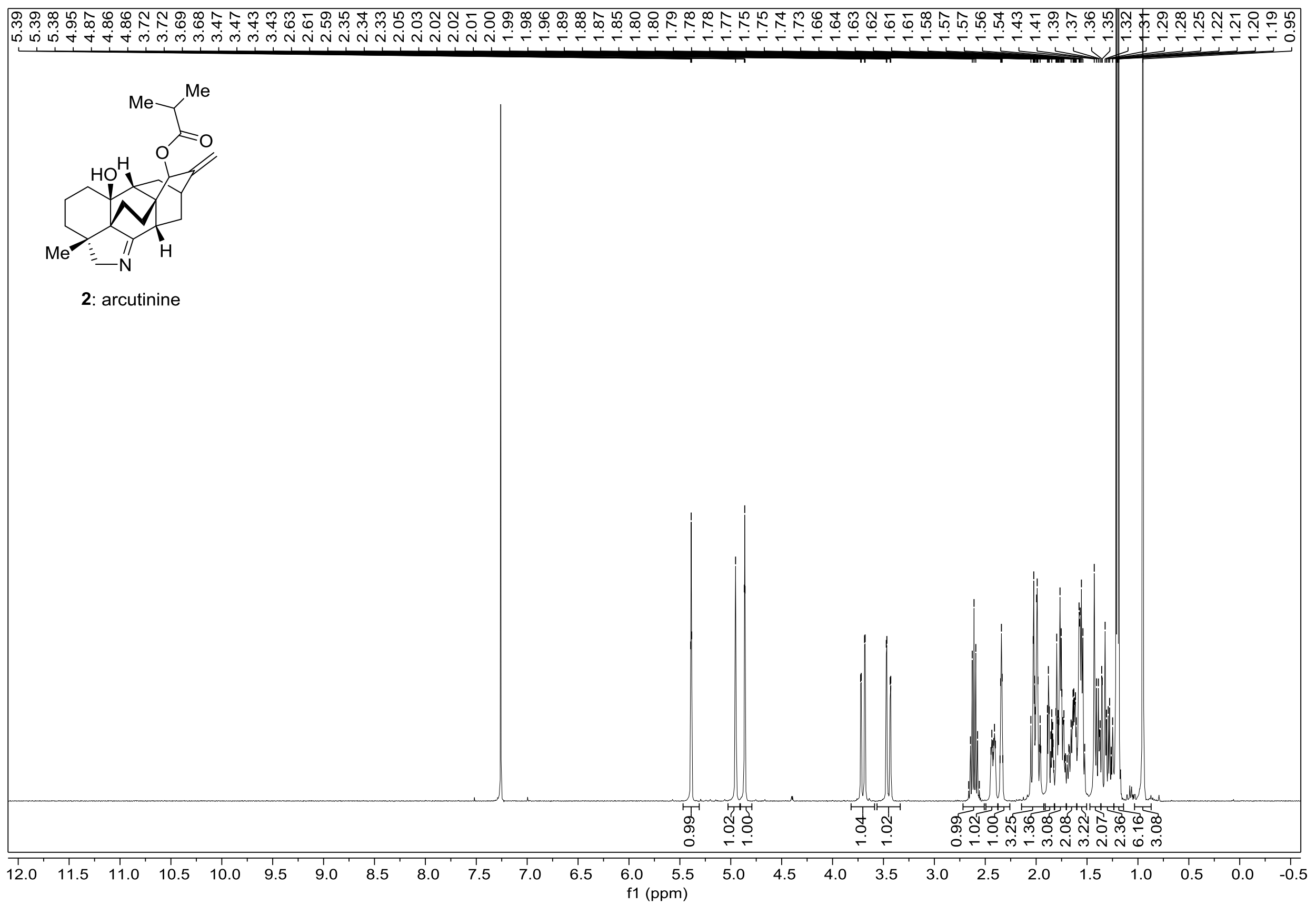


${ }^{13} \mathrm{C}$ NMR Spectrum of $2\left(126 \mathrm{MHz}, \mathrm{CDCl}_{3}\right)$

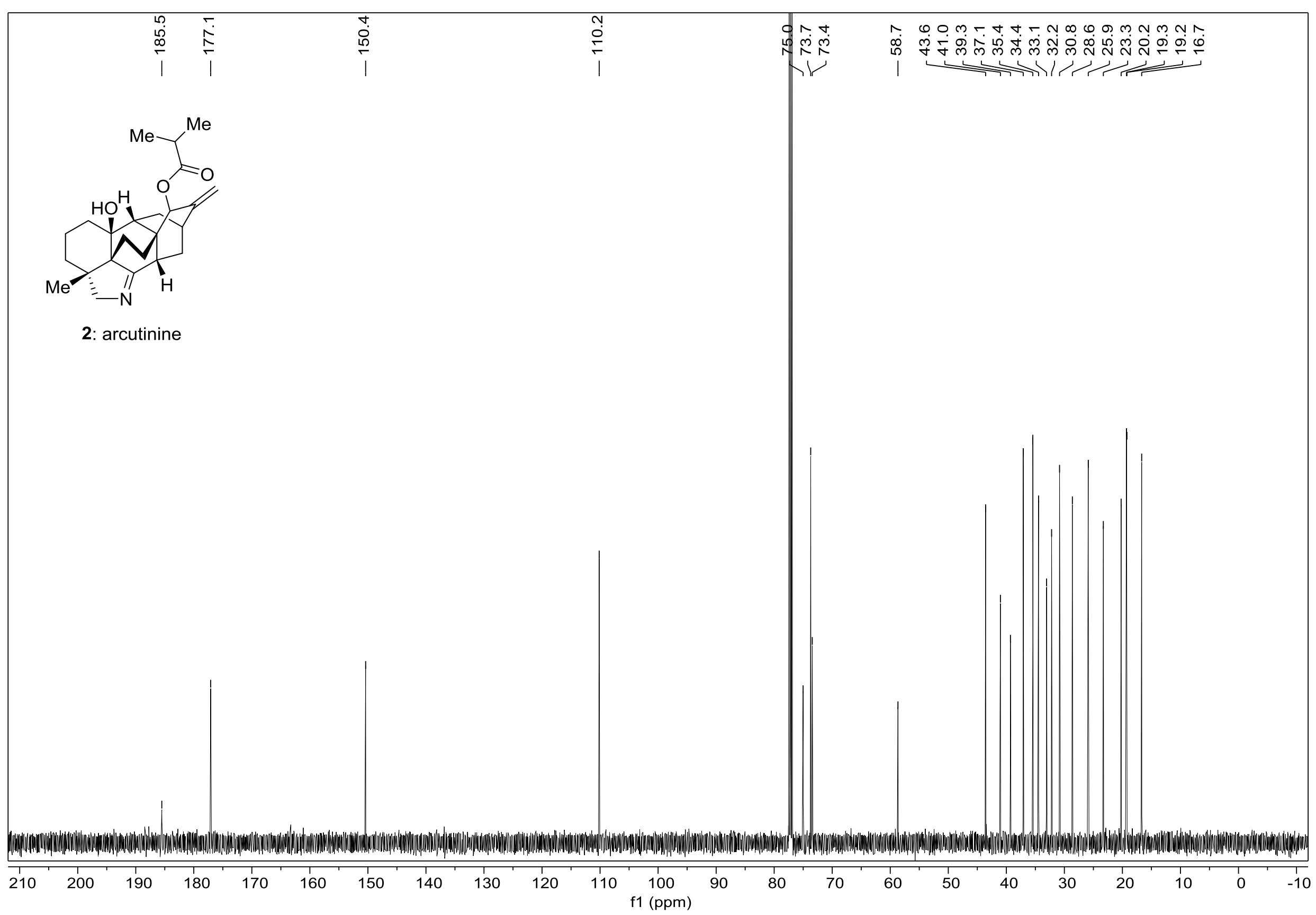




\section{${ }^{1} \mathrm{H}$ NMR Spectrum of 1 (400 $\left.\mathrm{MHz}, \mathrm{CDCl}_{3}\right)$}

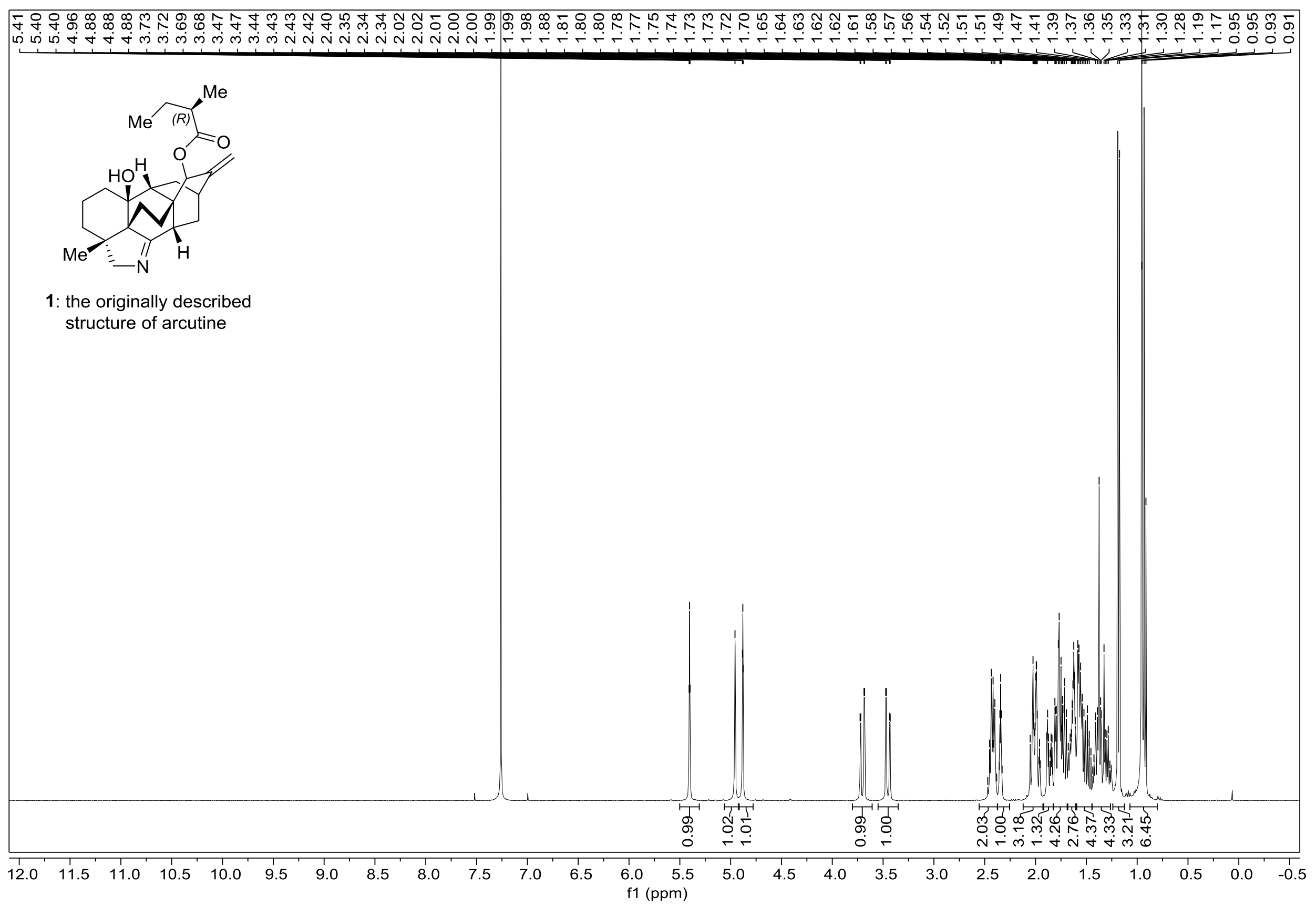


${ }^{13} \mathrm{C}$ NMR Spectrum of $1\left(151 \mathrm{MHz}, \mathrm{CDCl}_{3}\right)$

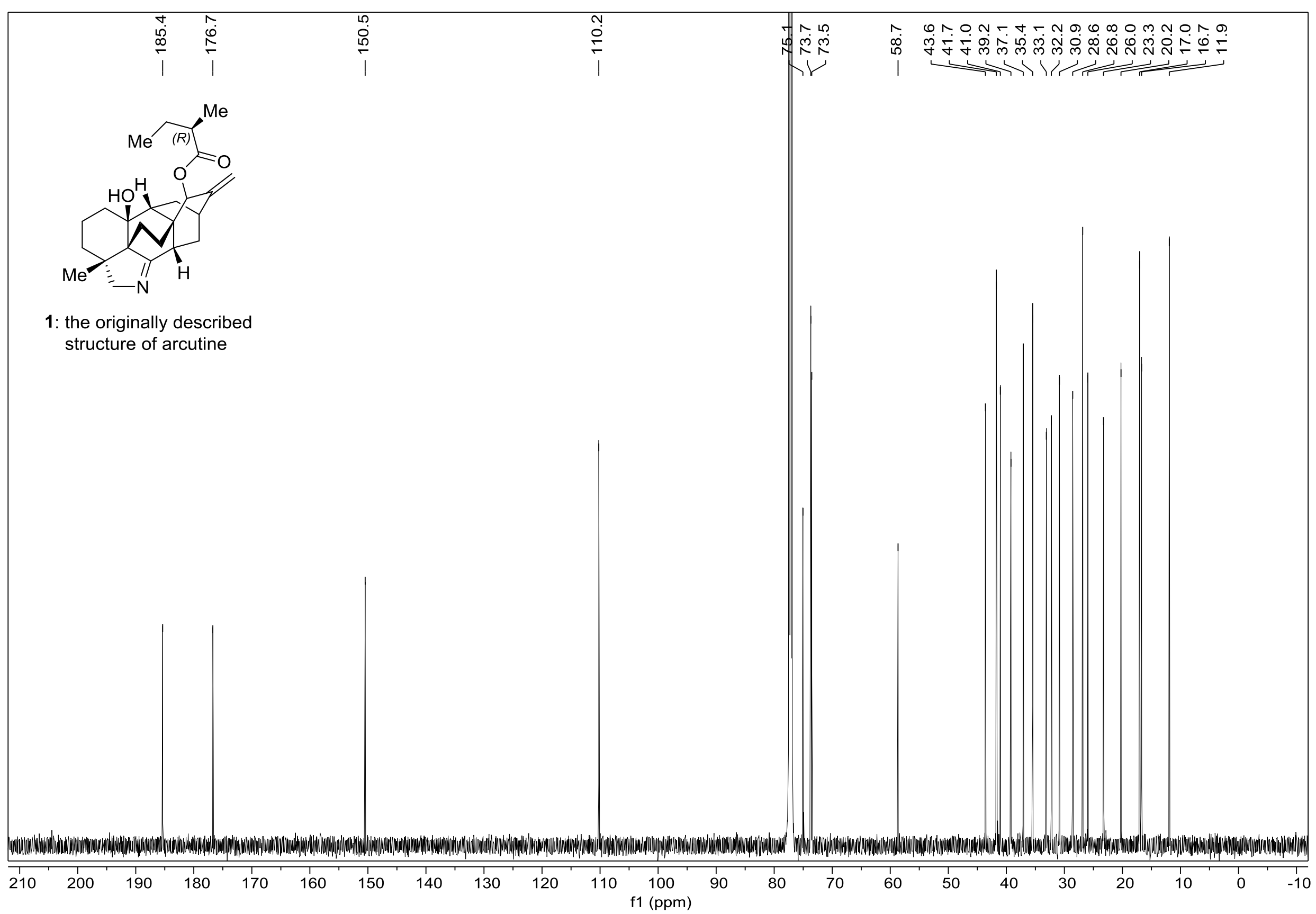




\section{${ }^{1} \mathrm{H}$ NMR Spectrum of $25\left(400 \mathrm{MHz}, \mathrm{CDCl}_{3}\right)$}

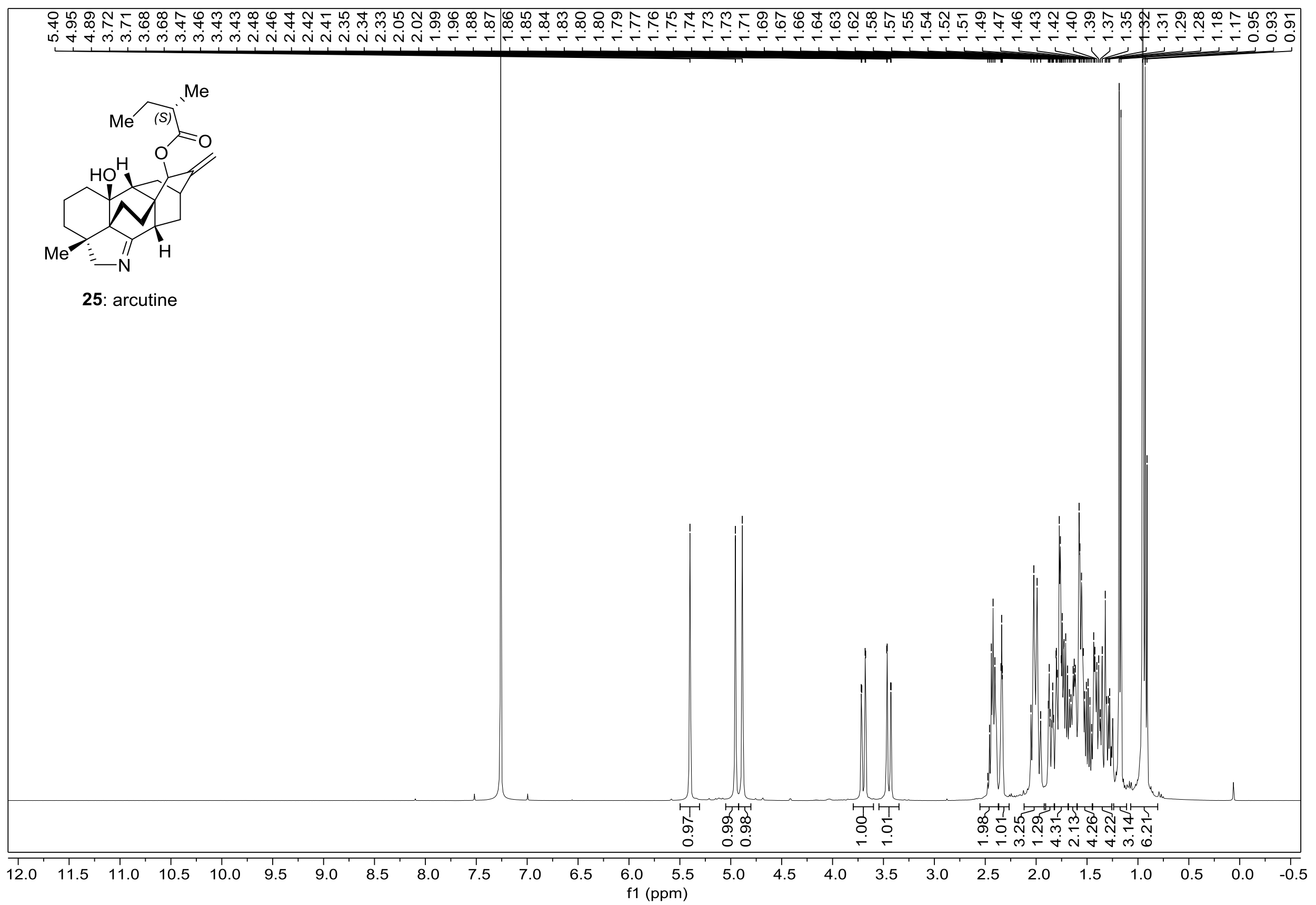


${ }^{13} \mathrm{C}$ NMR Spectrum of 25 (101 $\left.\mathrm{MHz}, \mathrm{CDCl}_{3}\right)$

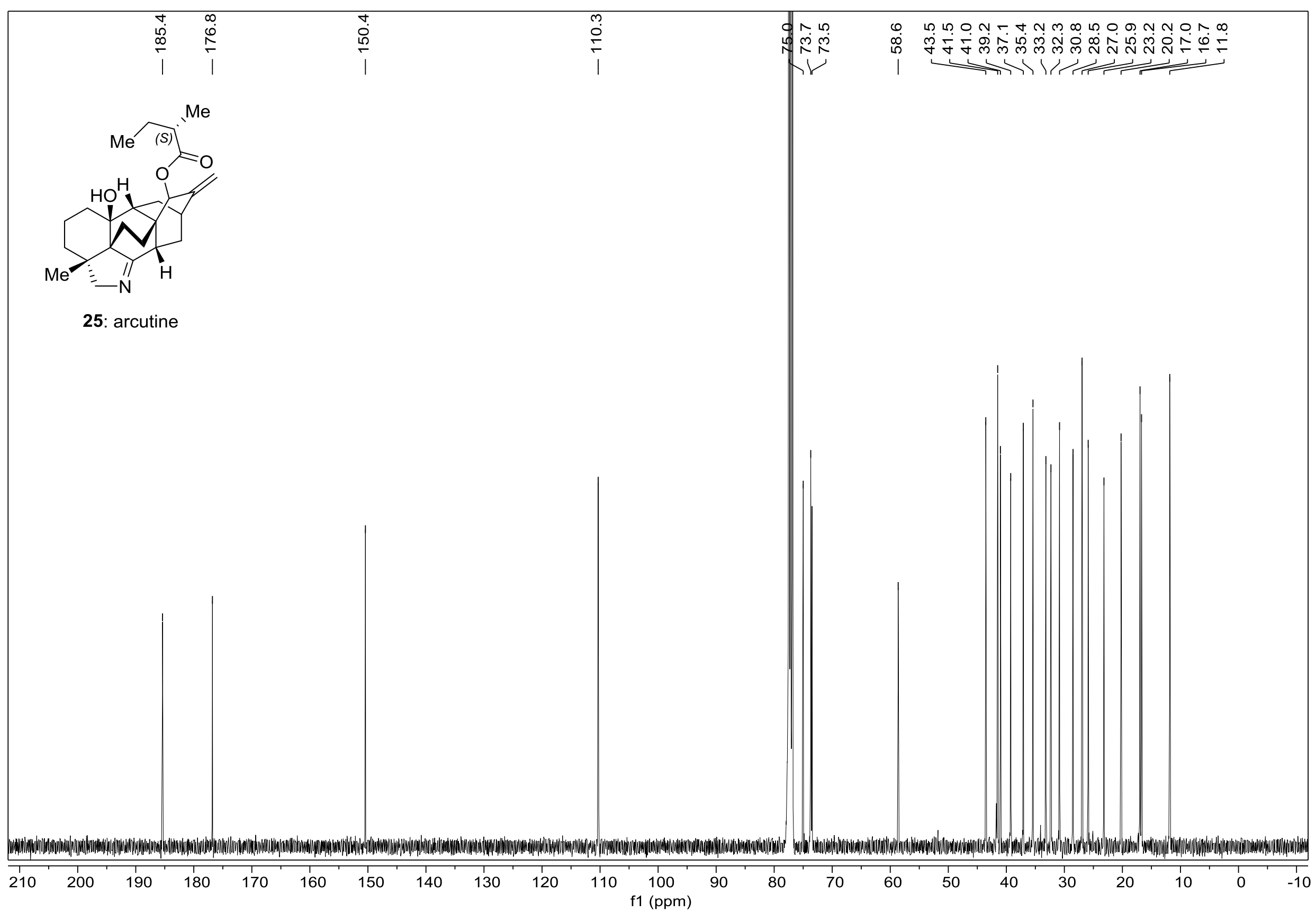


IV Comparison of the Spectroscopic Data of Authentic and Synthetic Arcutinidine

Table S1. Comparison of the ${ }^{13} \mathrm{C}$ NMR spectroscopic data $\left(\mathrm{CDCl}_{3}\right)$ of authentic and synthetic arcutinidine.

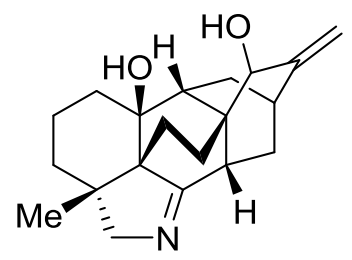

3: arcutinidine

\begin{tabular}{|c|c|c|}
\hline $\begin{array}{c}\text { Authentic } \\
\delta_{\mathrm{C}}(\mathrm{ppm})\end{array}$ & $\begin{array}{l}\text { Synthetic } \\
\delta_{\mathrm{C}}(\mathrm{ppm}) \\
126 \mathrm{MHz}\end{array}$ & $\begin{array}{c}\mathbf{E r r} \\
\text { (Authentic-Synthetic) } \\
\Delta \delta_{\mathrm{C}}(\mathrm{ppm})\end{array}$ \\
\hline 186.00 & 186.42 & -0.42 \\
\hline 155.31 & 155.37 & -0.06 \\
\hline 108.55 & 108.58 & -0.03 \\
\hline 75.06 & 75.07 & -0.01 \\
\hline 73.64 & 73.59 & +0.05 \\
\hline 73.26 & 73.31 & -0.05 \\
\hline 58.68 & 58.95 & -0.27 \\
\hline 42.53 & 42.58 & -0.05 \\
\hline 40.87 & 40.95 & -0.08 \\
\hline 40.19 & 40.30 & -0.11 \\
\hline 37.10 & 37.23 & -0.13 \\
\hline 35.35 & 35.41 & -0.06 \\
\hline 32.71 & 32.75 & -0.04 \\
\hline 31.85 & 31.85 & 0 \\
\hline 30.12 & 30.71 & -0.59 \\
\hline 28.25 & 28.90 & -0.65 \\
\hline 26.00 & 26.10 & -0.10 \\
\hline 23.43 & 23.57 & -0.14 \\
\hline 20.16 & 20.23 & -0.07 \\
\hline 16.55 & 16.65 & -0.10 \\
\hline
\end{tabular}




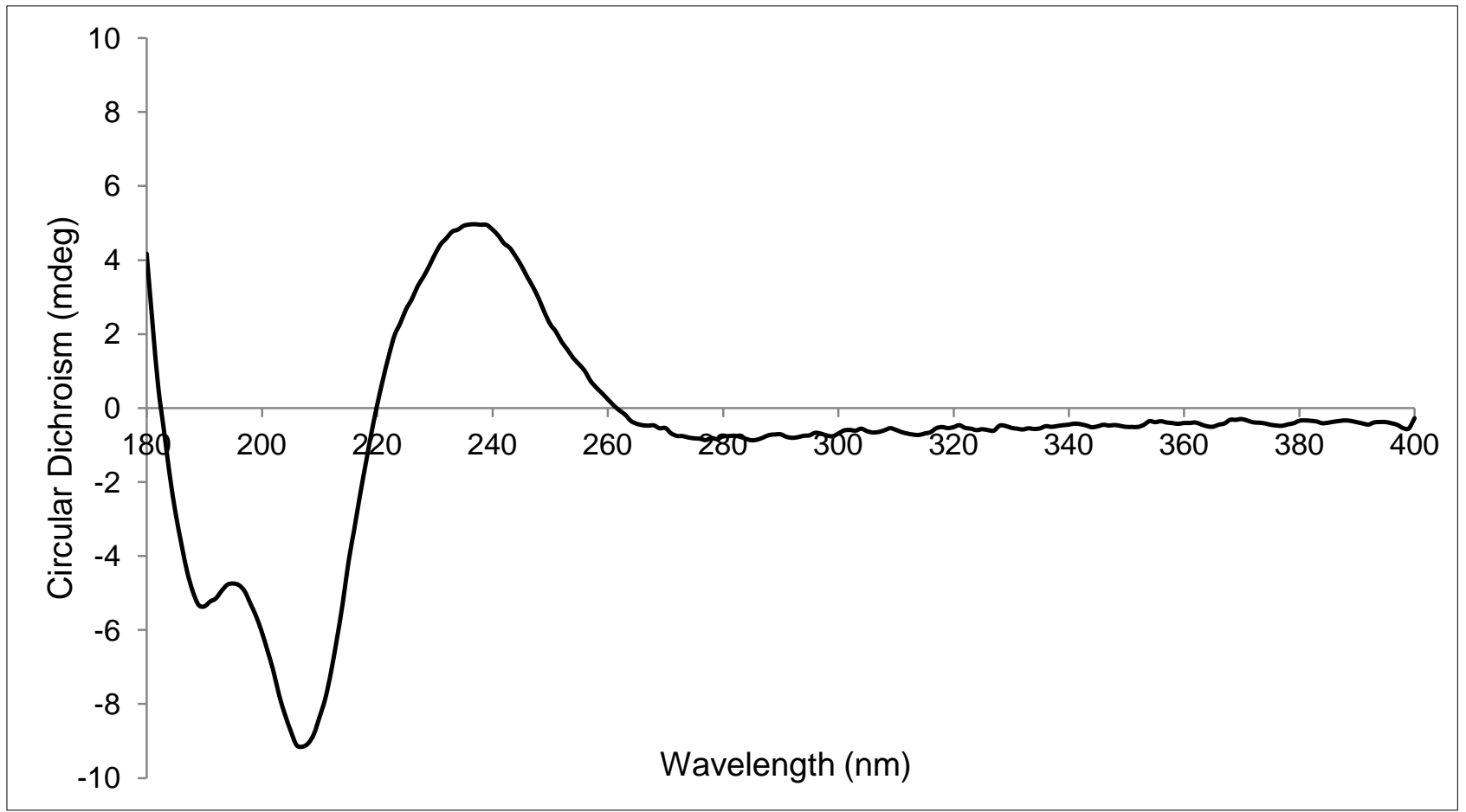

Figure S1. Circular dichroism spectrum of synthetic arcutinidine at $20{ }^{\circ} \mathrm{C}$. The mass concentration of arcutinidine in $\mathrm{MeCN}$ was $0.25 \mathrm{mg} / \mathrm{mL}$.

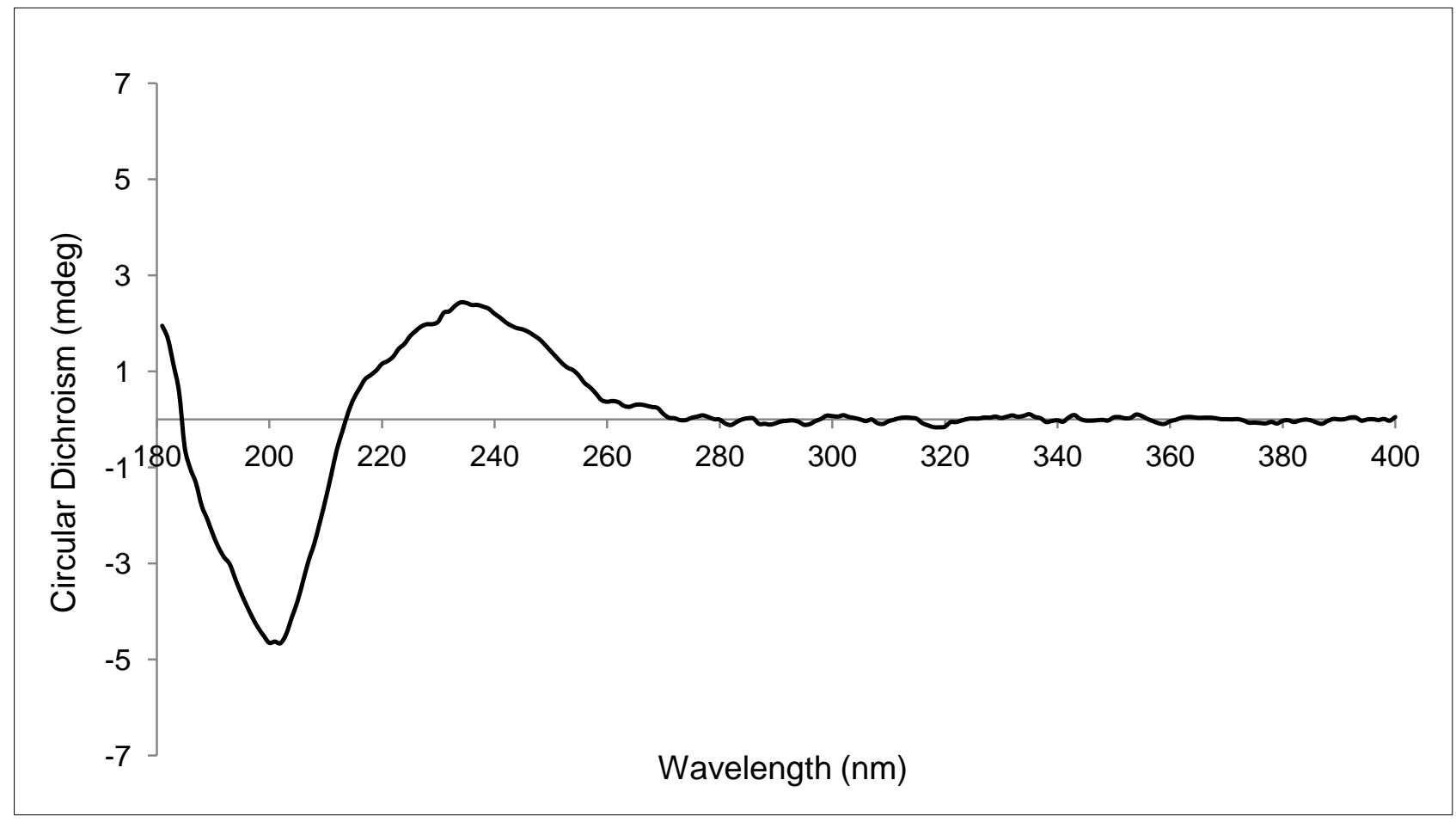

Figure S2. Circular dichroism spectrum of synthetic arcutine at $20{ }^{\circ} \mathrm{C}$. The mass concentration of arcutine in $\mathrm{MeCN}$ was $0.10 \mathrm{mg} / \mathrm{mL}$. 
(1) (a) Carrea, G.; Danieli, B.; Palmisano, G.; Riva, S.; Santagostino, M. Lipase-mediated resolution of 2-cyclohexen-1-ols as chiral buildingblocks en route to eburnane alkaloids. Tetrahedron: Asymmetry 1992, 3, 775. (b) Attolini, M.; Bouguir, F.; Iacazio, G.; Peiffer, G.; Maffei, M. Enantioselective synthesis of cyclic dialkyl (3-hydroxy-1-alkenyl) phosphonates by baker's yeast-mediated reduction of the corresponding enones. Tetrahedron 2001, 57, 537.

(2) Shih, C.; Swenton, J. S. Use of protected $\beta$-bromocyclopentenones and $\beta$-bromocyclohexenones as $\beta$-acylvinyl anion equivalents. J. Org. Chem. 1982, 47, 2825.

(3) (a) O’Byrne, A.; Murray, C.; Keegan, D.; Palacio, C.; Evans, P.; Morgan, B. S. The thio-adduct facilitated, enzymatic kinetic resolution of 4-hydroxycyclopentenone and 4-hydroxycyclohexenone. Org. Biomol. Chem. 2010, 8, 539. (b) Li, J.; Zhang, W.; Zhang, F.; Chen, Y.; Li, A. Total synthesis of daphniyunnine C (longeracinphyllin A). J. Am. Chem. Soc. 2017, 139, 14893.

(4) Carreño, M. C.; Urbano, A.; Di Vitta, C. Enantioselective Diels-Alder approach to C3-oxygenated angucyclinones from (SS)-2-(p-tolylsulfinyl)-1,4-naphthoquinone. Chem. - Eur. J. 2000, 6, 906.

(5) Seyferth, D.; Weiner, M. A. The preparation of organolithium compounds by the transmetalation reaction. I. vinyllithium. J. Am. Chem. Soc. 1961, 83, 3583.

(6) Ratcliffe, R.; Rodehorst, R. Improved procedure for oxidations with the chromium trioxide-pyridine complex. J. Org. Chem. 1970, 35, 4000.

(7) Tashkhodzhaev, B.; Saidkhodzhaeva, S. A.; Bessonova, I. A.; Antipin, M. Y. Arcutin-a new type of diterpene alkaloids. Chem. Nat. Compd. 2000, 36, 79. 